\title{
Iron-Catalyzed Tandem Three-Component Alkylation: Access to a-Methylated Substituted Ketones
}

Léo Bettoni, ${ }^{[a]}$ Charlotte Seck, ${ }^{[a, b]}$ Mbaye Diagne Mbaye, ${ }^{[a, b]}$ Sylvain Gaillard, ${ }^{[a]}$ Jean-Luc Renaud*[a]

${ }^{a}$ Normandie Univ, LCMT, ENSICAEN, UNICAEN, CNRS, 6 boulevard du Maréchal Juin, 14000 Caen, France.

${ }^{\mathrm{b} U n i v e r s i t e ́ ~ A s s a n e ~ S e c k ~ d e ~ Z i g u i n c h o r . ~ B P ~ 523, ~ Z i g u i n c h o r, ~ S e ́ n e ́ g a l . ~}$

Supporting Information

\section{Table of contents}

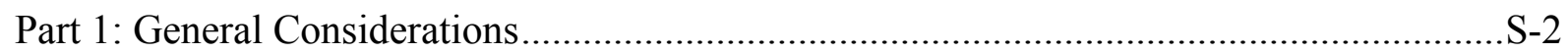

Part 2: Optimization conditions of 4-methoxyacetophenone alkylation ...............................S-3

Part 3: General procedures for the alkylation of Ketones (Three component reactions) .........S-4

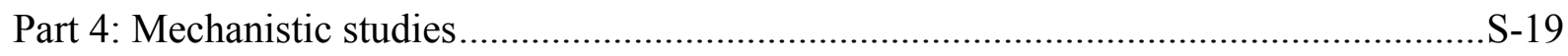

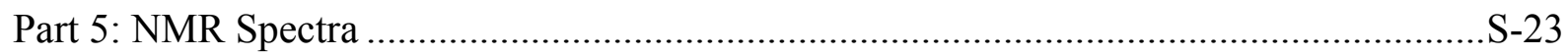




\section{Part 1: General Considerations}

All air- and moisture-sensitive manipulations were carried out using standard vacuum line Schlenk tubes techniques. Dry toluene was dried using a solvent purification system from Innovative Technologies, by passage through towers containing activated alumina. Xylene was purchased from Carlo Reba and was distillated over sodium and stocked over $4 \AA$ molecular sieves. Both and dioxane, dimethyl sulfoxide, dimethyl carbonate, tert-butanol and alcohol substrat were deglazed prior to use by bubbling argon gas directly in the solvent. Other solvents and chemicals were purchased from different suppliers and used as received. Neutral alumina was purchased from Alfa Aesar (Brockmann Grade I, 58 Angstroms, -60

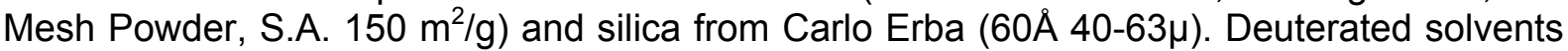
for NMR spectroscopy were purchased from Sigma Aldrich and used as received. NMR spectra were recorded on a $500 \mathrm{MHz}$ Brücker spectrometer. Proton $\left({ }^{1} \mathrm{H}\right)$ NMR information is given in the following format: multiplicity ( $s$, singlet; $d$, doublet; $t$, triplet; q, quartet; $m$, multiplet), coupling constant(s) $(J)$ in Hertz $(\mathrm{Hz})$, number of protons, type. The prefix app is occasionally applied when the true signal multiplicity was unresolved and $b r$ indicates the signal in question broadened. Carbon $\left({ }^{13} \mathrm{C}\right)$ NMR spectra are reported in ppm $(\delta)$ relative to $\mathrm{CDCl}_{3}$ unless noted otherwise. Infrared spectra were recorded over a PerkinElmer Spectrum 100 FT-IR Spectrometer using neat conditions. HRMS analyses were performed by Laboratoire de Chimie Moléculaire et Thioorganique analytical Facilities. 


\section{Part 2: Optimization conditions of 4-methoxyacetophenone alkylation}

\section{Table S1: Optimization of the reaction conditions ${ }^{a}$}<smiles>COc1ccc(CC(C)C(=O)c2ccc(CCC(=O)c3ccc(OC)cc3)cc2)cc1</smiles>
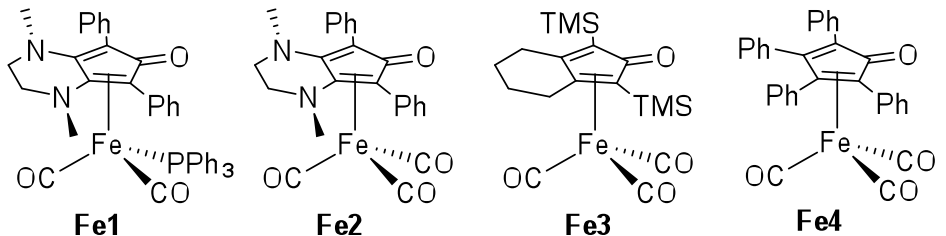

\begin{tabular}{|c|c|c|c|c|c|c|c|}
\hline entry & $\begin{array}{c}\text { Benzyl } \\
\text { alcohol/methanol } \\
(\mathrm{mL})\end{array}$ & catalyst & $\begin{array}{l}\text { temperature } \\
\left({ }^{\circ} \mathrm{C}\right)\end{array}$ & time $(\mathrm{h})$ & $1 a^{a}(\%)$ & $2^{a}(\%)$ & $3^{a}(\%)$ \\
\hline 1 & $0.5 / 0.5$ & Fe1 & 80 & 16 & 34 & 54 & 12 \\
\hline 2 & $0.5 / 0.5$ & Fe1 & 90 & 16 & 64 & 26 & 10 \\
\hline 3 & $0.5 / 0.5$ & Fe1 & 100 & 16 & 52 & 29 & 19 \\
\hline 4 & $0.265 / 0.735$ & Fe1 & 80 & 16 & 31 & 37 & 32 \\
\hline 5 & $0.265 / 0.735$ & Fe1 & 90 & 16 & 56 & 27 & 17 \\
\hline 6 & $0.265 / 0.735$ & Fe1 & 90 & 60 & 71 & 18 & 11 \\
\hline 7 & $0.065 / 0.5$ & Fe1 & 90 & 48 & 11 & 56 & 33 \\
\hline 8 & $0.065 / 1.0$ & Fe1 & 90 & 48 & 8 & 48 & 44 \\
\hline 9 & $0.265 / 0.5$ & Fe1 & 90 & 48 & 61 & 29 & 10 \\
\hline $10^{b}$ & $0.265 / 0.5$ & Fe1 & 90 & 48 & 39 & 58 & 3 \\
\hline $11^{\mathrm{c}}$ & $0.265 / 0.5$ & Fe1 & 90 & 48 & 26 & 69 & 5 \\
\hline 12 & $0.265 / 0.735$ & Fe1 & 100 & 48 & 40 & 29 & 31 \\
\hline 13 & $0.265 / 0.5$ & Fe1 & 100 & 48 & 46 & 31 & 23 \\
\hline 14 & $0.265 / 1.0$ & Fe1 & 90 & 48 & 69 & - & 31 \\
\hline $15^{\mathrm{d}}$ & $0.265 / 0.735$ & Fe1 & 90 & 48 & 12 & 75 & 13 \\
\hline $16^{d}$ & $0.265 / 1.0$ & Fe1 & 90 & 48 & 47 & 43 & 10 \\
\hline $17^{\mathrm{e}}$ & $0.265 / 1.0$ & Fe2 & 90 & 48 & 59 & 19 & 22 \\
\hline $18^{\mathrm{e}}$ & $0.265 / 0.735$ & Fe2 & 90 & 48 & 54 & 30 & 16 \\
\hline $19^{e}$ & $0.265 / 0.5$ & $\mathrm{Fe} 2$ & 90 & 48 & 55 & 32 & 13 \\
\hline $20^{f}$ & $0.265 / 1.0$ & Fe2 & 90 & 40 & 65 & - & 35 \\
\hline
\end{tabular}




\begin{tabular}{|c|c|c|c|c|c|c|c|}
\hline $21^{\dagger}$ & $0.265 / 0.735$ & Fe2 & 90 & 40 & 83 & - & 17 \\
\hline $22^{\dagger}$ & $0.265 / 0.5$ & Fe2 & 90 & 40 & 91 & - & 9 \\
\hline $23^{9}$ & $0.265 / 0.5$ & Fe2 & 90 & 40 & 91 & - & 9 \\
\hline $24^{9}$ & $0.265 / 0.3$ & Fe2 & 90 & 60 & 80 & 11 & 9 \\
\hline $25^{9}$ & $0.265 / 0.5$ & Fe3 & 90 & 40 & 20 & 19 & 61 \\
\hline $26^{9}$ & $0.265 / 0.5$ & Fe4 & 90 & 40 & - & 20 & - \\
\hline
\end{tabular}

[a] Conversion was determined by ${ }^{1} \mathrm{H}-\mathrm{NMR}$ analysis of the crude mixture. [b]: Reaction performed with 1 equiv of $\mathrm{K}_{3} \mathrm{PO}_{4}$. [c] Reaction performed with 0.5 equiv of $\mathrm{K}_{3} \mathrm{PO}_{4}$. [d]: Reaction in two steps: First addition of benzyl alcohol (5 equiv) in $1 \mathrm{~mL}$ of toluene with $\mathrm{Cs}_{2} \mathrm{CO}_{3}(10 \mathrm{~mol} \%)$, and then addition of methanol with $\mathrm{K}_{3} \mathrm{PO}_{4}$ (2 equiv). [e] Reaction performed in $1 \mathrm{~mL}$ of toluene with Pre-catalyst activated with $\mathrm{Me}_{3} \mathrm{NO}(3 \mathrm{~mol} \%)$. [f] Pre-catalyst was activated with $\mathrm{CsOH}(10 \mathrm{~mol} \%)$. [g] Pre-catalyst was activated with $\mathrm{KOH}(10 \mathrm{~mol} \%)$.

\section{Part 3: General procedures for the alkylation of Ketones (Three component reactions)}

\section{General Procedure A:}

In a $15 \mathrm{~mL}$ flame-dried Schlenk tube equipped with a stirring bar, the desired ketone $(0.5$ mmol, 1 equiv), benzyl alcohol ( $0.265 \mathrm{~mL}, 5$ equiv), $\mathrm{KOH}(2.8 \mathrm{mg}, 10 \mathrm{~mol} \%)$, methanol (25 equiv, $0.5 \mathrm{~mL}$ ), iron complex Fe2 (4.56 mg, $2 \mathrm{~mol} \%$ ) and $\mathrm{K}_{3} \mathrm{PO}_{4}(212 \mathrm{mg}, 2$ equiv) were poured in under an argon atmosphere. The mixture was rapidly stirred at room temperature for $2 \mathrm{~min}$ and then placed into a pre-heated oil bath at $90^{\circ} \mathrm{C}$ and stirred over 24 hours. The mixture was cooled-down to room temperature, filtrated over silica with diethyl ether and concentrated under reduced pressure. The conversion was determined by ${ }^{1} \mathrm{H}-\mathrm{NMR}$ spectroscopy, and then, the residue was purified by flash chromatography on silica gel using pentane-ethyl acetate as eluent to afford the desired product.

\section{General Procedure B:}

In a $15 \mathrm{~mL}$ flame-dried Schlenk tube equipped with a stirring bar, acetophenone $(0.5 \mathrm{mmol}$, $58 \mu \mathrm{L}, 1$ equiv), the desired alcohol (5 equiv), $\mathrm{KOH}(2.8 \mathrm{mg}, 10 \mathrm{~mol} \%)$, methanol (25 equiv, $0.5 \mathrm{~mL})$, iron complex Fe2 (4.56 mg, $2 \mathrm{~mol} \%)$ and $\mathrm{K}_{3} \mathrm{PO}_{4}(212 \mathrm{mg}, 2$ equiv) were poured in under an argon atmosphere. The mixture was rapidly stirred at room temperature for 2 min and then placed into a pre-heated oil bath at $90^{\circ} \mathrm{C}$ and stirred over 24 hours. The mixture was cooled-down to room temperature, filtrated over silica with diethyl ether and concentrated under reduced pressure. The conversion was determined by ${ }^{1} \mathrm{H}-\mathrm{NMR}$ spectroscopy, and then, the residue was purified by flash chromatography on silica gel using pentane-ethyl acetate as eluent to afford the desired product.

\section{General Procedure C:}

In a $15 \mathrm{~mL}$ flame-dried Schlenk tube equipped with a stirring bar, 2-acetylfuran $(0.5 \mathrm{mmol}, 55$ $\mathrm{mg}, 1$ equiv), the desired alcohol (5 equiv), $\mathrm{KOH}(2.8 \mathrm{mg}, 10 \mathrm{~mol} \%)$, methanol (25 equiv, 0.5 $\mathrm{mL}$ ), iron complex Fe2 (4.56 mg, $2 \mathrm{~mol} \%$ ) and $\mathrm{K}_{3} \mathrm{PO}_{4}(212 \mathrm{mg}, 2$ equiv) were poured in under an argon atmosphere. The mixture was rapidly stirred at room temperature for 2 min and then placed into a pre-heated oil bath at $90^{\circ} \mathrm{C}$ and stirred over 24 hours. The mixture was cooled-down to room temperature, filtrated over silica with diethyl ether and concentrated under reduced pressure. The conversion was determined by ${ }^{1} \mathrm{H}-\mathrm{NMR}$ spectroscopy, and then, the residue was purified by flash chromatography on silica gel using pentane-ethyl acetate as eluent to afford the desired product. 


\section{General Procedure D:}

In a $15 \mathrm{~mL}$ flame-dried Schlenk tube equipped with a stirring bar, 1-(4-\{[(2,2-dimethoxyethyl)(methyl)amino]methyl\}phenyl)ethan-1-one ${ }^{1}(0.5 \mathrm{mmol}, 126 \mathrm{mg}, 1$ equiv), the desired alcohol ( 5 equiv), $\mathrm{KOH}(2.8 \mathrm{mg}, 10 \mathrm{~mol} \%$ ), methanol ( 25 equiv, $0.5 \mathrm{~mL}$ ), iron complex Fe2 (4.56 mg, $2 \mathrm{~mol} \%)$ and $\mathrm{K}_{3} \mathrm{PO}_{4}(212 \mathrm{mg}, 2$ equiv) were poured in under an argon atmosphere. The mixture was rapidly stirred at room temperature for 2 min and then placed into a pre-heated oil bath at $90^{\circ} \mathrm{C}$ and stirred over 24 hours. The mixture was cooleddown to room temperature, filtrated over silica with diethyl ether and concentrated under reduced pressure. The conversion was determined by ${ }^{1} \mathrm{H}$-NMR spectroscopy, and then, the residue was purified by flash chromatography on silica gel using pentane-ethyl acetate as eluent to afford the desired product.<smiles>COc1ccc(C(=O)C(C)Cc2ccccc2)cc1</smiles>

Chemical Formula: $\mathrm{C}_{17} \mathrm{H}_{18} \mathrm{O}_{2}$

\section{- 2-methyl-3-phenyl-1-(4-methoxyphenyl)propan-1-one ${ }^{2}$ (1a)}

According to general procedure $A$, alkylation of $p$-methoxyacetophenone $(0.5 \mathrm{mmol}, 75 \mathrm{mg})$ afforded the pure product $1 \mathrm{a}$ as a colorless oil $(76 \mathrm{mg}, 60 \%)$ by silica flash column chromatography (pentane/ethyl acetate 95:5). ${ }^{1} \mathrm{H}-\mathrm{NMR}\left(\mathrm{CDCl}_{3}, \mathbf{5 0 0} \mathbf{~ M H z}\right): \delta 7.83$ (d, J = 8.5 $\mathrm{Hz}, 2 \mathrm{H}), 7.20-7.18(\mathrm{~m}, 2 \mathrm{H}), 7.13-7.10(\mathrm{~m}, 3 \mathrm{H}), 6.82(\mathrm{~d}, J=8.5 \mathrm{~Hz}, 2 \mathrm{H}), 3.78(\mathrm{~s}, 3 \mathrm{H}), 3.59$ (app sext, $J=7.0 \mathrm{~Hz}, 1 \mathrm{H}$ ), 3.05 (dd, $J=14.0 ; 6.5 \mathrm{~Hz}, 1 \mathrm{H}), 2.58(\mathrm{dd}, J=13.5 ; 7.0 \mathrm{~Hz}, 1 \mathrm{H}$ ), $1.10(\mathrm{~d}, J=7.0 \mathrm{~Hz}, 3 \mathrm{H}) \mathrm{ppm} .{ }^{13} \mathrm{C}-\mathrm{NMR}\left(\mathrm{CDCl}_{3}, 125 \mathrm{MHz}\right): \delta 202.3,163.4,140.2,130.6(2 \mathrm{C})$, $129.4,129.1$ (2C), 128.4 (2C),126.2, 113.8 (2C), 55.5, 42.4, 39.6, 17.6 ppm.

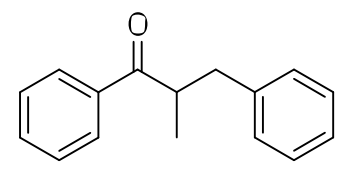

Chemical Formula: $\mathrm{C}_{16} \mathrm{H}_{16} \mathrm{O}$

\section{- 2-methyl-1,3-diphenylpropan-1-one ${ }^{2}$ (1b)}

According to general procedure $\mathrm{A}$, alkylation of acetophenone $(0.5 \mathrm{mmol}, 58 \mu \mathrm{L})$ afforded the pure product $1 \mathrm{~b}$ as a colorless oil $(67 \mathrm{mg}, 60 \%)$ by silica flash column chromatography (pentane/ethyl acetate 95:5). The conversion was determined by ${ }^{1} \mathrm{H}-\mathrm{NMR}$ spectroscopy in the crude product with $13 \%$ of dimethylated derivative observed. ${ }^{1} \mathbf{H}-\mathbf{N M R}\left(\mathbf{C D C l}_{3}, \mathbf{5 0 0} \mathbf{M H z}\right)$ : $\delta 7.90(\mathrm{~d}, J=7.0 \mathrm{~Hz}, 2 \mathrm{H}), 7.51(\mathrm{t}, J=7.0 \mathrm{~Hz}, 1 \mathrm{H}), 7.41(\mathrm{t}, J=7.5 \mathrm{~Hz}, 2 \mathrm{H}), 7.27-7.23(\mathrm{~m}, 2 \mathrm{H})$, 7.19-7.15 (m, 3H), 3.69 (app sext, $J=7.0 \mathrm{~Hz}, 1 \mathrm{H}), 3.13$ (dd, $J=13.7 ; 6.3 \mathrm{~Hz}, 1 \mathrm{H}), 2.65$ (dd, $J=13.7 ; 7.9 \mathrm{~Hz}, 1 \mathrm{H}), 1.17(\mathrm{~d}, J=7.0 \mathrm{~Hz}, 3 \mathrm{H})$ ppm. ${ }^{13} \mathbf{C}-\mathbf{N M R}\left(\mathbf{C D C l}_{3}, \mathbf{1 2 5} \mathbf{M H z}\right): \delta 203.8$, 140.0, 136.5, 133.0, 129.1 (2C), 128.7 (2C), 128.4 (2C), 128.3 (2C), 126.2, 42.8, 39.4, 17.4 ppm.

\footnotetext{
${ }^{1}$ Lator, A.; Gaignard Gaillard, Q.; Mérel, D.; Lohier, J-F.; Gaillard, S.; Poater, A.; Renaud, J-L.

${ }^{2}$ Chakrabarti, K.; Maji, M.; Panja, D.; Paul, B.; Sujan, S.; Gourab Kanti, D.; Kundu, S. Org. Lett. 2017, 19, 4750 4753.
} 
<smiles>CC(Cc1ccccc1)C(=O)c1ccc(C(F)(F)F)cc1</smiles>

Chemical Formula: $\mathrm{C}_{17} \mathrm{H}_{15} \mathrm{~F}_{3} \mathrm{O}$

\section{- 2-methyl-3-phenyl-1-(4-(trifluoromethyl)phenyl)propan-1-one (1c)}

According to general procedure A, alkylation of 4-trifluoromethylacetophenone $(0.5 \mathrm{mmol}, 94$ $\mathrm{mg}$ ) afforded the pure product $1 \mathrm{c}$ as a colorless oil $(80 \mathrm{mg}, 55 \%)$ by silica flash column chromatography (pentane/ethyl acetate 95:5). The conversion was determined by ${ }^{1} \mathrm{H}-\mathrm{NMR}$ spectroscopy in the crude product with $14 \%$ of dimethylated derivative observed. ${ }^{1} \mathbf{H}-\mathbf{N M R}$ $\left(\mathrm{CDCl}_{3}, 500 \mathrm{MHz}\right): \delta 7.90(\mathrm{~d}, J=8.0 \mathrm{~Hz}, 2 \mathrm{H}), 7.61(\mathrm{~d}, J=8.0 \mathrm{~Hz}, 2 \mathrm{H}), 7.21-7.18(\mathrm{~m}, 2 \mathrm{H})$, 7.13-7.10 (m, 3H), 3.63 (app sext, $J=7.0 \mathrm{~Hz}, 1 \mathrm{H}), 3.07$ (dd, $J=14 ; 7.0 \mathrm{~Hz}, 1 \mathrm{H}), 2.63$ (dd, $J$ $=14 ; 7.0 \mathrm{~Hz}, 1 \mathrm{H}), 1.17(\mathrm{~d}, J=7.0 \mathrm{~Hz}, 3 \mathrm{H})$ ppm. ${ }^{19} \mathrm{~F}-\mathrm{NMR}\left(\mathbf{C D C l}_{3}, 500 \mathbf{~ M H z}\right): \delta-63.09 \mathrm{ppm}$. ${ }^{13}$ C-NMR (CDCl $\left.3,125 \mathrm{MHz}\right): \delta 202.91,139.56,134.4,129.06(2 \mathrm{C}), 128.55\left(\mathrm{~d}, J^{C-F}=11.7 \mathrm{~Hz}\right)$, 126.4, $125.72\left(q, J^{C-F}=3.8 \mathrm{~Hz}\right), 43.30,39.32,17.35$ ppm. IR (neat): $v$ 3029, 2974, 2934, 1687, 1581, 1510, 1454, 1322, 1167, 1126, 1066, 975, 853, 697, 593, $518 \mathrm{~cm}^{-1}$. HRMS $[\mathrm{M}+\mathrm{H}]^{+}$: calculated $\mathrm{C}_{17} \mathrm{H}_{16} \mathrm{~F}_{3} \mathrm{O}$ 293.1153; found: 293.1153 .

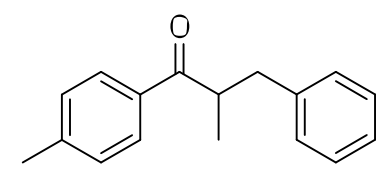

Chemical Formula: $\mathrm{C}_{17} \mathrm{H}_{18} \mathrm{O}$

\section{- 2-methyl-3-phenyl-1-(p-tolyl)propan-1-one ${ }^{2}$ (1d)}

According to general procedure $A$, alkylation of $p$-methylacetophenone $(0.5 \mathrm{mmol}, 67 \mu \mathrm{L})$ afforded the pure product $1 \mathrm{~d}$ as a colorless oil $(92 \mathrm{mg}, 70 \%)$ by silica flash column chromatography (pentane/ethyl acetate 95:5). The conversion was determined by ${ }^{1} \mathrm{H}-\mathrm{NMR}$ spectroscopy in the crude product with $9 \%$ of dimethylated derivative observed. ${ }^{1} \mathbf{H}-\mathbf{N M R}$ $\left(\mathrm{CDCl}_{3}, \mathbf{5 0 0} \mathrm{MHz}\right): \delta 7.75(\mathrm{~d}, J=8.5 \mathrm{~Hz}, 2 \mathrm{H}), 7.19-7.15(\mathrm{~m}, 4 \mathrm{H}), 7.12-7.08(\mathrm{~m}, 3 \mathrm{H}), 3.61$ (app sext, $J=7.0 \mathrm{~Hz}, 1 \mathrm{H}$ ), $3.06(\mathrm{dd}, J=14 ; 6 \mathrm{~Hz}, 1 \mathrm{H}), 2.58(\mathrm{dd}, J=14 ; 7.5 \mathrm{~Hz}, 1 \mathrm{H}), 2.31(\mathrm{~s}$, $3 \mathrm{H}), 1.10(\mathrm{~d}, J=7.0 \mathrm{~Hz}, 3 \mathrm{H}) \mathrm{ppm} .{ }^{13} \mathrm{C}-\mathrm{NMR}\left(\mathrm{CDCl}_{3}, 125 \mathrm{MHz}\right): \delta 203.4,143.7,140.1,134.0$, 129.4 (2C), 129.1 (2C), 128.5 (2C), 128.4 (2C), 126.2, 42.6, 39.4, 21.7, 17.5 ppm.

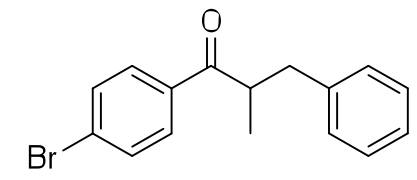

Chemical Formula: $\mathrm{C}_{16} \mathrm{H}_{15} \mathrm{BrO}$

\section{- 2-methyl-3-phenyl-1-(4-bromophenyl)propan-1-one ${ }^{2}$ (1e)}

According to general procedure $A$, alkylation of $p$-bromoacetophenone $(0.5 \mathrm{mmol}, 99.5 \mathrm{mg})$ afforded the pure product 1e as a colorless oil $(62 \mathrm{mg}, 41 \%)$ by silica flash column chromatography (pentane/ethyl acetate 95:5). The conversion was determined by ${ }^{1} \mathrm{H}-\mathrm{NMR}$ spectroscopy in the crude product with $19 \%$ of dimethylated derivative observed. ${ }^{1} \mathbf{H}-\mathbf{N M R}$ $\left(\mathrm{CDCl}_{3}, 500 \mathrm{MHz}\right): \delta 7.76(\mathrm{~d}, J=8.5 \mathrm{~Hz}, 2 \mathrm{H}), 7.57(\mathrm{~d}, J=8.5 \mathrm{~Hz}, 2 \mathrm{H}), 7.26-7.24(\mathrm{~m}, 2 \mathrm{H})$, 7.19-7.17 (m, 3H), 3.64 (app sext, $J=7.0 \mathrm{~Hz}, 1 \mathrm{H}$ ), 3.12 (dd, $J=13.7 ; 6.3 \mathrm{~Hz}, 1 \mathrm{H}$ ), 2.67 (dd, $J=13.7 ; 7.5 \mathrm{~Hz}, 1 \mathrm{H}), 1.19$ (d, $J=7.0 \mathrm{~Hz}, 3 \mathrm{H})$ ppm. ${ }^{13} \mathbf{C}-\mathrm{NMR}\left(\mathrm{CDCl}_{3}, 125 \mathrm{MHz}\right): \delta 202.8$, 139.7, 135.2, 132.0 (2C), 129.8 (2C), 129.0 (2C), 128.5 (2C), 128.1, 126.3, 42.9, 39.4, 17.5 ppm. 


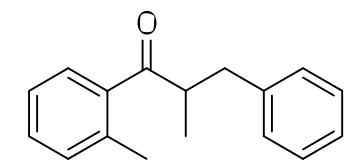

Chemical Formula: $\mathrm{C}_{17} \mathrm{H}_{18} \mathrm{O}$

\section{- 2-methyl-3-phenyl-1-(o-tolyl)propan-1-one (1f)}

According to general procedure $\mathrm{A}$, alkylation of o-methylacetophenone $(0.5 \mathrm{mmol}, 65 \mu \mathrm{L})$ afforded the pure product $1 \mathrm{f}$ as a colorless oil $(108 \mathrm{mg}, 91 \%)$ by silica flash column chromatography (pentane/ethyl acetate 95:5). The conversion was determined by ${ }^{1} \mathrm{H}-\mathrm{NMR}$ spectroscopy in the crude product with $3 \%$ of dimethylated derivative observed. ${ }^{1} \mathbf{H}-\mathbf{N M R}$ $\left(\mathrm{CDCl}_{3}, 500 \mathrm{MHz}\right): \delta 7.39(\mathrm{~d}, J=8.0 \mathrm{~Hz}, 1 \mathrm{H}), 7.30(\mathrm{td}, J=7.5 ; 1.5 \mathrm{~Hz}, 1 \mathrm{H}), 7.27-7.24(\mathrm{~m}$, 2H), 7.22-7.17 (m, 5H), 3.51 (app sext, $J=7.0 \mathrm{~Hz}, 1 \mathrm{H}$ ), 3.13 (dd, $J=13.7 ; 6.4 \mathrm{~Hz}, 1 \mathrm{H}$ ), 2.61 (dd, $J=13.7 ; 7.5 \mathrm{~Hz}, 1 \mathrm{H}), 2.39(\mathrm{~s}, 3 \mathrm{H}), 1.14(\mathrm{~d}, J=7.0 \mathrm{~Hz}, 3 \mathrm{H})$ ppm. ${ }^{13} \mathrm{C}-\mathrm{NMR}\left(\mathrm{CDCl}_{3}, 125\right.$ MHz): $\delta 208.2,140.0,138.6,137.8,131.7,130.8,129.1$ (2C), 128.4 (2C), 127.6, 126.2, 125.6, 46.3, 39.2, 20.8, 17.0 ppm. IR (neat): v 3062, 2972, 2901, 1699, 1587, 1453, 1427, $1373,1222,1053,970,892,698,553,494 \mathrm{~cm}^{-1}$. HRMS [M+H] : calculated $\mathrm{C}_{17} \mathrm{H}_{19} \mathrm{O}$ 239.1436; found: 239.1433 .

\section{- Scale up of the reaction to $5 \mathrm{mmol}$ starting material}

In a $30 \mathrm{~mL}$ flame-dried Schlenk tube equipped with a stirring bar, o-methylacetophenone (5 $\mathrm{mmol}, 0.65 \mathrm{~mL})$, benzyl alcohol $(25 \mathrm{mmol}, 2.65 \mathrm{~mL}), \mathrm{KOH}(10 \mathrm{~mol} \%, 28.1 \mathrm{mg})$, methanol (25 equiv, $5 \mathrm{~mL}$ ), iron complex Fe2 (2 mol \%, $45.6 \mathrm{mg})$ and K3PO4 (10 mmol, 2.12g) were poured in under an argon atmosphere. The mixture was rapidly stirred at room temperature for $2 \mathrm{~min}$ and then placed into a pre-heated oil bath at $90^{\circ} \mathrm{C}$ and stirred over 24 hours. The mixture was cooled-down to room temperature, filtrated over silica with diethyl ether and concentrated under reduced pressure. Then the residue was purified by flash chromatography on silica gel using pentane-ethyl acetate (95:5) as eluent to afford the pure product $1 \mathrm{f}$ as a colorless oil $(1.075 \mathrm{~g}, 90 \%) .{ }^{1} \mathrm{H}-\mathrm{NMR}$ data was comparable with the previous NMR data.

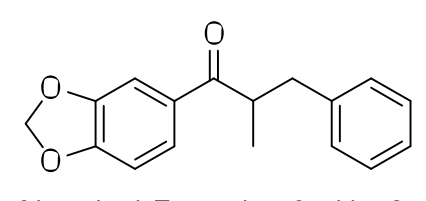

Chemical Formula: $\mathrm{C}_{17} \mathrm{H}_{16} \mathrm{O}_{3}$

\section{- 2-methyl-3-phenyl-1-(benzo[d][1,3]dioxol-5-yl)propan-1-one ${ }^{2}(1 \mathrm{~g})$}

According to general procedure A, alkylation of 3,4-(methylenedioxy)acetophenone (0.5 $\mathrm{mmol}, 82 \mathrm{mg}$ ) afforded the pure product $1 \mathrm{~g}$ as a colorless oil $(70 \mathrm{mg}, 52 \%)$ by silica flash column chromatography (pentane/ethyl acetate 95:5). The conversion was determined by ${ }^{1} \mathrm{H}-$ NMR spectroscopy in the crude product with $12 \%$ of dimethylated derivative observed. ${ }^{1} \mathrm{H}$ NMR $\left(\mathrm{CDCl}_{3}, \mathbf{5 0 0} \mathrm{MHz}\right): \delta 7.51(\mathrm{dd}, J=8.0 ; 1.5 \mathrm{~Hz}, 1 \mathrm{H}), 7.41(\mathrm{~d}, J=1.5 \mathrm{~Hz}, 1 \mathrm{H}), 7.28-7.25$ $(\mathrm{m}, 3 \mathrm{H}), 7.19-7.17(\mathrm{~m}, 2 \mathrm{H}), 6.82(\mathrm{~d}, J=8.0 \mathrm{~Hz}, 1 \mathrm{H}), 6.04(\mathrm{~s}, 2 \mathrm{H}), 3.62(\operatorname{app~sext}, J=7.0 \mathrm{~Hz}$, $1 \mathrm{H}), 3.12(\mathrm{dd}, J=13.7 ; 6.3 \mathrm{~Hz}, 1 \mathrm{H}), 2.66(\mathrm{dd}, J=13.7 ; 7.4 \mathrm{~Hz}, 1 \mathrm{H}), 1.18(\mathrm{~d}, J=7.0 \mathrm{~Hz}, 3 \mathrm{H})$ ppm. ${ }^{13}$ C-NMR $\left(\mathrm{CDCl}_{3}, 125 \mathrm{MHz}\right): \delta 201.8,151.7,148.3,140.0,131.3,129.1$ (2C), 128.4 (2C), 126.2, 124.4, 108.2, 107.9, 101.9, 42.6, 39.60, 17.7 ppm. 
<smiles>CC(Cc1ccccc1)C(=O)c1ccc2ccccc2c1</smiles>

Chemical Formula: $\mathrm{C}_{20} \mathrm{H}_{18} \mathrm{O}$

\section{- 2-methyl-3-phenyl-1-(naphthalen-2-yl)propan-1-one ${ }^{2}$ (1h)}

According to general procedure $A$, alkylation of 2-acetylnaphtalene $(0.5 \mathrm{mmol}, 85 \mathrm{mg})$ afforded the pure product $1 \mathrm{~h}$ as a colorless oil $(74 \mathrm{mg}, 54 \%)$ by silica flash column chromatography (pentane/ethyl acetate 95:5). The conversion was determined by ${ }^{1} \mathrm{H}-\mathrm{NMR}$ spectroscopy in the crude product with $15 \%$ of dimethylated derivative observed. ${ }^{1} \mathbf{H}-\mathbf{N M R}$ $\left(\mathrm{CDCl}_{3}, 500 \mathrm{MHz}\right): \delta 8.34(\mathrm{~s}, 1 \mathrm{H}), 7.93(\mathrm{dd}, J=8.5 ; 1.5 \mathrm{~Hz}, 1 \mathrm{H}), 7.86(\mathrm{~d}, J=8.0 \mathrm{~Hz}, 1 \mathrm{H})$, 7.79 (t, $J=8.5 \mathrm{~Hz}, 2 \mathrm{H}), 7.46$ (td, $J=25 ; 7.5 \mathrm{~Hz}, 2 \mathrm{H}), 7.22-7.16(\mathrm{~m}, 4 \mathrm{H}), 7.09(\mathrm{t}, J=7.0 \mathrm{~Hz}$, $1 \mathrm{H}$ ), 3.81 (app sext, $J=7.0 \mathrm{~Hz}, 1 \mathrm{H}$ ), $3.14(\mathrm{dd}, J=13.7 ; 6.4 \mathrm{~Hz}, 1 \mathrm{H}), 2.67$ (dd, $J=13.7 ; 7.4$ $\mathrm{Hz}, 1 \mathrm{H}), 1.19$ (d, $J=7.0 \mathrm{~Hz}, 3 \mathrm{H})$ ppm. ${ }^{13} \mathbf{C}-\mathbf{N M R}\left(\mathrm{CDCl}_{3}, 125 \mathrm{MHz}\right): \delta$ 203.8, 140.1, 135.5, 133.8, 132.6, 129.8, 129.6, 129.2 (2C), 128.5, 128.4 (2C), 126.8, 126.3, 124.3, 117.2, 42.9, 39.6, $17.7 \mathrm{ppm}$.

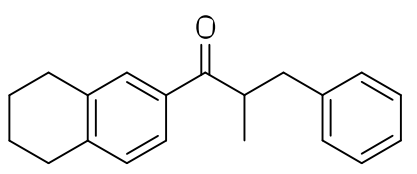

Chemical Formula: $\mathrm{C}_{20} \mathrm{H}_{22} \mathrm{O}$

\section{- 2-methyl-3-phenyl-1-(5,6,7,8-tetrahydronaphthalen-2-yl)propan-1-one (1i)}

According to general procedure A, alkylation of 1-(5,6,7,8-tetrahydronaphthalen-2-yl)ethan-1one $(0.5 \mathrm{mmol}, 82 \mu \mathrm{L})$ afforded the pure product $1 \mathrm{i}$ as a colorless oil $(77 \mathrm{mg}, 55 \%)$ by silica flash column chromatography (pentane/ethyl acetate 95:5). The conversion was determined by ${ }^{1} \mathrm{H}$-NMR spectroscopy in the crude product with $15 \%$ of dimethylated derivative observed. ${ }^{1} \mathrm{H}-\mathrm{NMR}\left(\mathrm{CDCl}_{3}, \mathbf{5 0 0} \mathrm{MHz}\right): \delta$ 7.63-7.61 (m, 2H), 7.25-7.22 (m, 2H), 7.18-7.13 (m, 3H), 7.09 (d, $J=8.0 \mathrm{~Hz}, 1 \mathrm{H}), 3.65$ (app sext, $J=7.0 \mathrm{~Hz}, 1 \mathrm{H}), 3.11$ (dd, $J=13.7 ; 6.3 \mathrm{~Hz}, 1 \mathrm{H}), 2.78-2.76$ $(\mathrm{m}, 4 \mathrm{H}), 2.62(\mathrm{dd}, J=13.7 ; 7.4 \mathrm{~Hz}, 1 \mathrm{H}), 1.79-1.77(\mathrm{~m}, 4 \mathrm{H}), 1.15(\mathrm{~d}, J=7.0 \mathrm{~Hz}, 3 \mathrm{H}) \mathrm{ppm} .{ }^{13} \mathrm{C}-$ NMR (CDCl 3,125 MHz): $\delta 203.7,143.1,140.2,137.5,133.9,129.4,129.3,129.2$ (2C), 128.4 (2C), 126.2, 125.4, 42.6, 39.4, 29.6, 29.5, 23.0, 22.9, 17.6 ppm. IR (neat): v 3062, 2971, 2928, 1675, 1603, 1570, 1495, 1453, 1248, 1228, 1077, 1066, 1057, 746, 699, $516 \mathrm{~cm}^{-1}$. HRMS [M+H] $]^{+}$calculated $\mathrm{C}_{20} \mathrm{H}_{22} \mathrm{O} 279.1749$; found: 279.1749 .

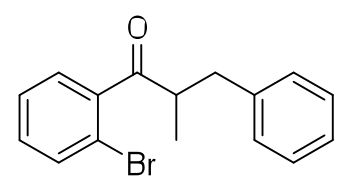

Chemical Formula: $\mathrm{C}_{16} \mathrm{H}_{15} \mathrm{BrO}$

\section{- 2-methyl-3-phenyl-1-(2-bromophenyl)propan-1-one (1j)}

According to general procedure $A$, alkylation of o-bromoacetophenone $(0.5 \mathrm{mmol}, 68 \mu \mathrm{L})$ afforded the pure product $1 \mathbf{j}$ as a colorless oil $(89 \mathrm{mg}, 59 \%)$ by silica flash column chromatography (pentane/ethyl acetate 95:5). The conversion was determined by ${ }^{1} \mathrm{H}-\mathrm{NMR}$ spectroscopy in the crude product with $21 \%$ of dimethylated derivative observed. ${ }^{1} \mathbf{H}-\mathbf{N M R}$ $\left(\mathrm{CDCl}_{3}, \mathbf{5 0 0} \mathrm{MHz}\right): \delta 7.51$ (dd, $\left.J=8.0 ; 1.5 \mathrm{~Hz}, 1 \mathrm{H}\right), 7.25-7.17(\mathrm{~m}, 4 \mathrm{H}), 7.14-7.11(\mathrm{~m}, 3 \mathrm{H})$, 7.07 (dd, $J=8.0 ; 1.5 \mathrm{~Hz}, 1 \mathrm{H}), 3.43(\operatorname{app}$ sext, $J=7.0 \mathrm{~Hz}, 1 \mathrm{H}), 3.10$ (dd, $J=8.5 ; 5.8 \mathrm{~Hz}, 1 \mathrm{H}$ ), 2.55 (dd, $J=13.7 ; 7.4 \mathrm{~Hz}, 1 \mathrm{H}), 1.08(\mathrm{~d}, J=7.0 \mathrm{~Hz}, 3 \mathrm{H})$ ppm. ${ }^{13} \mathrm{C}-\mathrm{NMR}\left(\mathbf{C D C l}_{3}, 125 \mathrm{MHz}\right): \delta$ 207.7, 141.9, 139.6, 138.0, 133.5, 131.3, 129.2 (2C), 128.4 (2C), 127.3, 126.3, 118.9, 47.5, 
38.8, 16.2 ppm. IR (neat): v 3062, 2972, 2901, 1698, 1587, 1496, 1453, 1222, 1050, 970, $740,698,494 \mathrm{~cm}^{-1}$. HRMS [M+H] ${ }^{+}$: calculated $\mathrm{C}_{16} \mathrm{H}_{16} \mathrm{BrO} 303.0385$; found: 303.0386 .

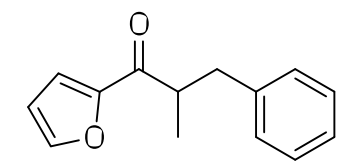

Chemical Formula: $\mathrm{C}_{14} \mathrm{H}_{14} \mathrm{O}_{2}$

\section{- 2-methyl-3-phenyl-1-(furan-2-yl)propan-1-one (1k)}

According to general procedure A, alkylation of 2-acetylfuran $(0.5 \mathrm{mmol}, 55 \mathrm{mg})$ afford the pure product $1 \mathrm{k}$ as a light yellow oil $(67 \mathrm{mg}, 63 \%)$ by silica flash column chromatography (pentane/ethyl acetate 95:5). The conversion was determined by ${ }^{1} \mathrm{H}-\mathrm{NMR}$ spectroscopy in the crude product with $8 \%$ of dimethylated derivative observed. ${ }^{1} \mathrm{H}-\mathrm{NMR}\left(\mathrm{CDCl}_{3}, \mathbf{5 0 0} \mathbf{M H z}\right)$ : $\delta 7.56(\mathrm{~d}, J=1.0 \mathrm{~Hz}, 1 \mathrm{H}), 7.27-7.24(\mathrm{~m}, 2 \mathrm{H}), 7.20-7.16(\mathrm{~m}, 3 \mathrm{H}), 7.14(\mathrm{~d}, J=6.5 \mathrm{~Hz}, 1 \mathrm{H})$, 6.50 (dd, $J=3.5 ; 1.0 \mathrm{~Hz}, 1 \mathrm{H}), 3.48($ app sext, $J=7.0 \mathrm{~Hz}, 1 \mathrm{H}), 3.11$ (dd, $J=13.7 ; 6.4 \mathrm{~Hz}$, $1 \mathrm{H}), 2.66(\mathrm{dd}, J=13.7 ; 7.9 \mathrm{~Hz}, 1 \mathrm{H}), 1.19(\mathrm{~d}, J=7.0 \mathrm{~Hz}, 3 \mathrm{H}) \mathrm{ppm} .{ }^{13} \mathrm{C}-\mathrm{NMR}\left(\mathrm{CDCl}_{3}, 125\right.$ MHz): $\delta 192.7,152.4,146.4,139.8,129.1$ (2C), 128.4 (2C), 126.2, 117.4, 112.2, 43.7, 39.2, 16.9 ppm. IR (neat): v 3133, 2972, 2901, 1670, 1566, 1465, 1256, 1080, 1014, 980, 883, 756, 698, 593, 509, $494 \mathrm{~cm}^{-1}$. HRMS [M+H] $]^{+}$: calculated $\mathrm{C}_{14} \mathrm{H}_{15} \mathrm{O}_{2} 215.1072$; found: 215.1073 .<smiles>Cc1cc(C)c(C(=O)C(C)Cc2ccccc2)c(C)c1</smiles>

\section{- 2-methyl-3-phenyl-1-(mesityl)propan-1-one ${ }^{3}$ (1l)}

According to general procedure A, alkylation of 1-mesitylethan-1-one $(0.5 \mathrm{mmol}, 83 \mu \mathrm{L})$ to afforded the pure product $1 \mathrm{l}$ as a colorless oil $(118 \mathrm{mg}, 89 \%)$ by silica flash column chromatography (pentane/ethyl acetate 95:5). The conversion was determined by ${ }^{1} \mathrm{H}-\mathrm{NMR}$ spectroscopy in the crude product with $4 \%$ of dimethylated derivative observed. ${ }^{1} \mathbf{H}-\mathbf{N M R}$ $\left(\mathrm{CDCl}_{3}, 500 \mathrm{MHz}\right) \delta$ 7.30-7.27 (m, 2H), 7.22-7.18 (m, 3H), $6.84(\mathrm{~s}, 2 \mathrm{H}), 3.22(\mathrm{dd}, J=13.5$; $5.0 \mathrm{~Hz}, 1 \mathrm{H}), 3.11(\mathrm{~m}, 1 \mathrm{H}), 2.55(\mathrm{dd}, J=13.5 ; 9.5 \mathrm{~Hz}, 1 \mathrm{H}), 2.28(\mathrm{~s}, 3 \mathrm{H}), 2.17(\mathrm{~s}, 6 \mathrm{H}), 1.08(\mathrm{~d}$, $J=7.0 \mathrm{~Hz}, 3 \mathrm{H}) \mathrm{ppm} .{ }^{13} \mathbf{C}-\mathbf{N M R}\left(\mathbf{C D C l}_{3}, 125 \mathbf{~ M H z}\right): \delta 213.0,139.9,138.5,133.5,129.3(2 \mathrm{C})$, 128.7 (2C), 128.3 (2C), 126.2, 49.8, 38.0, 21.1, 19.7 (2C), 15.5 ppm. IR (neat): $v 3027,2968$, 2922, 2732, 1690, 1610, 1496, 1453, 1225, 1153, 1030, 971, 850, 747, 699, $506 \mathrm{~cm}^{-1}$. HRMS [M+H] $]^{+}$: calculated $\mathrm{C}_{19} \mathrm{H}_{23} \mathrm{O} 267.1749$; found: 267.1743 .

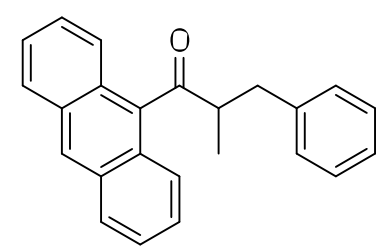

Chemical Formula: $\mathrm{C}_{24} \mathrm{H}_{20} \mathrm{O}$

- 2-methyl-3-phenyl-1-(anthracen-9-yl)propan-1-one (1m)

\footnotetext{
${ }^{3}$ Schlepphorst, C.; Maji, B.; Glorius, F. ACS Catal. 2016, 6, 4184-4188.
} 
According to general procedure A, alkylation of 1-(anthracen-9-yl)ethan-1-one $(0.5 \mathrm{mmol}$, $110 \mathrm{mg}$ ) afforded the pure product $1 \mathrm{~m}$ as a yellow oil (107 $\mathrm{mg}, 66 \%)$ by silica flash column chromatography (pentane/ethyl acetate 95:5). The conversion was determined by ${ }^{1} \mathrm{H}-\mathrm{NMR}$ spectroscopy in the crude product with $5 \%$ of dimethylated derivative observed. ${ }^{1} \mathbf{H}-\mathrm{NMR}$ $\left(\mathrm{CDCl}_{3}, 500 \mathrm{MHz}\right): \delta 8.50(\mathrm{~s}, 1 \mathrm{H}), 8.02(\mathrm{~d}, J=8.5 \mathrm{~Hz}, 2 \mathrm{H}), 7.50-7.44(\mathrm{~m}, 4 \mathrm{H}), 7.32-7.23(\mathrm{~m}$, 7H), 3.54 (app sext, $J=7.0 \mathrm{~Hz}, 1 \mathrm{H}$ ), $3.42(\mathrm{dd}, J=13.7 ; 6.4 \mathrm{~Hz}, 1 \mathrm{H}), 2.76(\mathrm{dd}, J=13.5 ; 7.0$ $\mathrm{Hz}, 1 \mathrm{H}), 1.20(\mathrm{~d}, J=7.0 \mathrm{~Hz}, 3 \mathrm{H}) \mathrm{ppm} .{ }^{13} \mathrm{C}-\mathrm{NMR}\left(\mathrm{CDCl}_{3}, 125 \mathrm{MHz}\right): \delta 212.5,139.8,135.7$, 134.2, 131.1, 129.5 (2C), 129.0, 128.8 (2C), 128.7, 128.5 (2C), 127.9, 127.3, 126.7 (2C), 126.4, 125.4 (2C), 124.8, 51.5, 38.5, 16.5 ppm. IR (neat): v 2971, 2901, 1733, 1689, 1671, $1601,1453,1313,1074,931,890,738,698,621 \mathrm{~cm}^{-1}$. HRMS [M+H] $]^{+}$calculated $\mathrm{C}_{24} \mathrm{H}_{21} \mathrm{O}$ 325.1592; found: 325.1588 .<smiles>CC(Cc1ccccc1)C(=O)c1ccccn1</smiles>

\section{- 2-methyl-3-phenyl-1-(pyridin-2-yl)propan-1-one (1n)}

According to general procedure $A$, alkylation of 2-acetylpyridine $(0.5 \mathrm{mmol}, 56 \mu \mathrm{L})$ afforded the pure product $1 \mathrm{n}$ as a colorless oil (38 $\mathrm{mg}, 33 \%$ ) by silica flash column chromatography (pentane/ethyl acetate 95:5). The conversion was determined by ${ }^{1} \mathrm{H}-\mathrm{NMR}$ spectroscopy in the crude product with $9 \%$ of dimethylated derivative observed. ${ }^{1} \mathbf{H}-\mathbf{N M R}\left(\mathbf{C D C l}_{3}, \mathbf{5 0 0} \mathbf{M H z}\right)$ : $\delta 8.67(\mathrm{~d}, J=5.0 \mathrm{~Hz}, 1 \mathrm{H}), 8.01(\mathrm{~d}, J=7.5 \mathrm{~Hz}, 1 \mathrm{H}), 7.79$ (t, $J=7.5 \mathrm{~Hz}, 1 \mathrm{H}), 7.42$ (dd, $J=7.5$; $5.0 \mathrm{~Hz}, 1 \mathrm{H}), 7.25-7.23(\mathrm{~m}, 4 \mathrm{H}), 7.16-7.14(\mathrm{~m}, 1 \mathrm{H}), 4.36$ (app sext, $J=7.0 \mathrm{~Hz}, 1 \mathrm{H}), 3.15$ (dd, $J=13.7 ; 6.4 \mathrm{~Hz}, 1 \mathrm{H}), 2.63(\mathrm{dd}, J=13.7 ; 7.9 \mathrm{~Hz}, 1 \mathrm{H}), 1.15(\mathrm{~d}, J=7.0 \mathrm{~Hz}, 3 \mathrm{H})$ ppm. ${ }^{13} \mathrm{C}-\mathrm{NMR}$ $\left(\mathrm{CDCl}_{3}, 125 \mathrm{MHz}\right): \delta 204.8,152.9,149.0,140.2,137.0,129.2$ (2C), 128.2 (2C), 127.0, 126.0, 122.5, 41.2, 39.0, 16.6 ppm. IR (neat): v 2971, 2901, 1695, 1583, 1496, 1453, 1225, 1076, 1066, 1057, 979, 850, 745, 699, $618 \mathrm{~cm}^{-1}$. HRMS [M+H] : calculated $\mathrm{C}_{15} \mathrm{H}_{16} \mathrm{NO} 226.1232$; found: 226.1228 .<smiles>CC(Cc1ccccc1)C(=O)CCc1ccccc1</smiles>

Chemical Formula: $\mathrm{C}_{18} \mathrm{H}_{20} \mathrm{O}$

\section{- 2-methyl-1,5-diphenylpentan-3-one ${ }^{4}$ (10)}

According to general procedure A, alkylation of 4-phenylbutan-2-one $(0.5 \mathrm{mmol}, 75 \mu \mathrm{L})$ afforded the pure product 10 as a colorless oil $(43 \mathrm{mg}, 34 \%)$ by silica flash column chromatography (pentane/ethyl acetate 95:5). The conversion was determined by ${ }^{1} \mathrm{H}-\mathrm{NMR}$ spectroscopy in the crude product with $44 \%$ of monobenzylated derivative observed. ${ }^{1} \mathrm{H}$ NMR (CDCl, 500 MHz): $\delta$ 7.26-7.22 (m, 4H), 7.19-7.14 (m, 2H), $7.08(\mathrm{~d}, J=7.0 \mathrm{~Hz}, 4 \mathrm{H})$, $2.91(\mathrm{dd}, J=13.7 ; 7.4 \mathrm{~Hz}, 1 \mathrm{H}), 2.82-2.75(\mathrm{~m}, 3 \mathrm{H}), 2.73-2.67(\mathrm{~m}, 1 \mathrm{H}), 2.57-2.51(\mathrm{~m}, 2 \mathrm{H}), 1.03$ $(\mathrm{d}, J=7.0 \mathrm{~Hz}, 3 \mathrm{H}) \mathrm{ppm} .{ }^{13} \mathrm{C}-\mathrm{NMR}\left(\mathrm{CDCl}_{3}, 125 \mathrm{MHz}\right): \delta 213.3,141.2,139.7,129.0(2 \mathrm{C})$, 128.5 (2C), 128.4 (2C), 126.3, 126.1, 48.3, 43.8, 39.2, 29.6, 16.4 ppm.

\footnotetext{
${ }^{4}$ Amani, J.; Molander, G. J. Org. Chem. 2017, 82, 1856 - 1863.
} 
<smiles>CC(C)C(=O)C(C)Cc1ccccc1</smiles>

Chemical Formula: $\mathrm{C}_{13} \mathrm{H}_{18} \mathrm{O}$

\section{- 2,4-dimethyl-1-phenylpentan-3-one ${ }^{5}(1 p)$}

According to general procedure A, alkylation of 3-methylbutan-2-one $(0.5 \mathrm{mmol}, 54 \mu \mathrm{L})$ afforded the pure product $1 \mathrm{p}$ as a colorless oil $(37 \mathrm{mg}, 39 \%)$ by silica flash column chromatography (pentane/ethyl acetate 95:5). The conversion was determined by ${ }^{1} \mathrm{H}-\mathrm{NMR}$ spectroscopy in the crude product with $47 \%$ of monobenzylated derivative observed. ${ }^{1} \mathrm{H}$ NMR (CDCl, 500 MHz): $\delta$ 7.27-7.24 (m, 2H), 7.20-7.16 (m, 1H), 7.14-7.12 (m, 2H), 3.00$2.92(\mathrm{~m}, 2 \mathrm{H}), 2.57-2.48(\mathrm{~m}, 2 \mathrm{H}), 1.07(\mathrm{~d}, J=6.5 \mathrm{~Hz}, 3 \mathrm{H}), 1.00(\mathrm{~d}, J=6.5 \mathrm{~Hz}, 3 \mathrm{H}), 0.86(\mathrm{~d}, J$ $=6.5 \mathrm{~Hz}, 3 \mathrm{H}) \mathrm{ppm} .{ }^{13} \mathrm{C}-\mathrm{NMR}\left(\mathrm{CDCl}_{3}, 125 \mathrm{MHz}\right): \delta 217.9,140.0,129.1$ (2C), $128.4(2 \mathrm{C})$, $126.2,46.6,40.5,39.6,18.0,17.7,17.2 \mathrm{ppm}$.<smiles>CC(Cc1ccccc1)C(=O)C1CC1</smiles>

Chemical Formula: $\mathrm{C}_{13} \mathrm{H}_{16} \mathrm{O}$

\section{- 1-cyclopropyl-2-methyl-3-phenylpropan-1-one ${ }^{2}$ (1q)}

According to general procedure $A$, alkylation of 1-cyclopropylethan-1-one (0.5 mmol, $47 \mu \mathrm{L})$ afforded the pure product $1 \mathrm{q}$ as a colorless oil $(48 \mathrm{mg}, 40 \%)$ by silica flash column chromatography (pentane/ethyl acetate 95:5). The conversion was determined by ${ }^{1} \mathrm{H}-\mathrm{NMR}$ spectroscopy in the crude product with $46 \%$ of monobenzylated derivative observed. ${ }^{1} \mathrm{H}$ NMR ( $\left.\mathrm{CDCl}_{3}, \mathbf{5 0 0} \mathbf{~ M H z}\right): \delta$ 7.23-7.18 (m, 2H), 7.15-7.10 (m, 3H), 2.97 (dd, J = 13.7; $6.3 \mathrm{~Hz}$, 1H), 2.87 (app sext, $J=7.0 \mathrm{~Hz}, 1 \mathrm{H}), 2.51(\mathrm{dd}, J=13.7 ; 7.4 \mathrm{~Hz}, 1 \mathrm{H}), 1.88-1.83(\mathrm{~m}, 1 \mathrm{H}), 1.06$ (d, $J=7.0 \mathrm{~Hz}, 3 \mathrm{H}), 0.94-0.86(\mathrm{~m}, 2 \mathrm{H}), 0.80-0.70(\mathrm{~m}, 2 \mathrm{H}) \mathrm{ppm} .{ }^{13} \mathbf{C}-\mathrm{NMR}\left(\mathbf{C D C l}_{3}, 125 \mathbf{~ M H z}\right): \delta$ 213.9, 139.9, 129.1 (2C), 128.4 (2C), 126.2, 49.0, 39.0, 19.7, 16.3, 11.0, 10.9 ppm.<smiles>CC(Cc1cccs1)C(=O)c1ccccc1</smiles>

Chemical Formula: $\mathrm{C}_{14} \mathrm{H}_{14} \mathrm{OS}$

\section{- 2-methyl-3-(thiophen-2-yl)-1-phenylpropan-1-one ${ }^{2}$ (1r)}

According to general procedure $\mathrm{B}$, alkylation of acetophenone with thiophen-2-ylmethanol ( $0.237 \mathrm{~mL}, 5$ equiv) afforded the pure product $1 \mathrm{r}$ as a light yellow oil ( $82 \mathrm{mg}, 72 \%$ ) by silica flash column chromatography (pentane/ethyl acetate 98:2). The conversion was determined by ${ }^{1} \mathrm{H}$-NMR spectroscopy in the crude product with $9 \%$ of dimethylated derivative observed. ${ }^{1} \mathrm{H}-\mathrm{NMR}\left(\mathrm{CDCl}_{3}, 500 \mathrm{MHz}\right): \delta 7.94(\mathrm{~d}, J=7.0 \mathrm{~Hz}, 2 \mathrm{H}), 7.54(\mathrm{t}, J=7.0 \mathrm{~Hz}, 1 \mathrm{H}), 7.45$ (t, $J=$ $7.5 \mathrm{~Hz}, 2 \mathrm{H}), 7.10(\mathrm{dd}, J=5.0 ; 1.0 \mathrm{~Hz}, 1 \mathrm{H}), 6.88(\mathrm{dd}, J=5.0 ; 3.2 \mathrm{~Hz}, 1 \mathrm{H}), 6.80(\mathrm{~d}, J=2.9 \mathrm{~Hz}$, $1 \mathrm{H}$ ), 3.74 (sext, $J=7.0 \mathrm{~Hz}, 1 \mathrm{H}$ ), 3.36 (dd, $J=15.0 ; 7.0 \mathrm{~Hz}, 1 \mathrm{H}$ ), 2.93 (dd, $J=15.0 ; 7.0 \mathrm{~Hz}$, $1 \mathrm{H}), 1.25(\mathrm{~d}, J=7.0 \mathrm{~Hz}, 3 \mathrm{H}) \mathrm{ppm} .{ }^{13} \mathrm{C}-\mathrm{NMR}\left(\mathrm{CDCl}_{3}, 125 \mathrm{MHz}\right): \delta 203.2,142.5,136.3,133.1$, $128.7(2 \mathrm{C}), 128.4(2 \mathrm{C}), 126.8,125.6,123.6,43.3,33.3,17.8 \mathrm{ppm}$.

\footnotetext{
${ }^{5}$ K. M Chan, L.; L Poole, Darren.; Shen D.; P Healy M.; J Donohoe, T. Angew. Chem. Int. Ed. 2014, 53, 761 765.
} 
<smiles>CC(Cc1ccco1)C(=O)c1ccccc1</smiles>

Chemical Formula: $\mathrm{C}_{14} \mathrm{H}_{14} \mathrm{O}_{2}$

\section{- 2-methyl-3-(furan-2-yl)-1-phenylpropan-1-one ${ }^{6}$ (1s)}

According to general procedure $B$, alkylation of acetophenone with furan-2-ylmethanol $(0.217$ $\mathrm{mL}, 5$ equiv) afforded the pure product $1 \mathrm{~s}$ as a yellow oil ( $82 \mathrm{mg}, 77 \%)$ by silica flash column chromatography (pentane/ethyl acetate 98:2). The conversion was determined by ${ }^{1} \mathrm{H}-\mathrm{NMR}$ spectroscopy in the crude product with $8 \%$ of dimethylated derivative observed. ${ }^{1} \mathbf{H}-\mathbf{N M R}$ $\left(\mathrm{CDCl}_{3}, 500 \mathrm{MHz}\right): \delta 7.93(\mathrm{~d}, J=7.0 \mathrm{~Hz}, 2 \mathrm{H}), 7.53(\mathrm{t}, J=7.5 \mathrm{~Hz}, 1 \mathrm{H}), 7.44(\mathrm{t}, J=7.5 \mathrm{~Hz}$, $2 \mathrm{H}), 7.27(\mathrm{~d}, J=1.5 \mathrm{~Hz}, 1 \mathrm{H}), 6.22(\mathrm{dd}, J=2.8 ; 1.7 \mathrm{~Hz}, 1 \mathrm{H}), 6.00(\mathrm{~d}, J=2.5 \mathrm{~Hz}, 1 \mathrm{H}), 3.82$ (sext, $J=7.0 \mathrm{~Hz}, 1 \mathrm{H}$ ), 3.11 (dd, $J=15.0 ; 6.3 \mathrm{~Hz}, 1 \mathrm{H}$ ), 2.73 (dd, $J=15.0 ; 7.4 \mathrm{~Hz}, 1 \mathrm{H}$ ), 1.20 (d, $J=7.0 \mathrm{~Hz}, 3 \mathrm{H})$ ppm. ${ }^{13} \mathrm{C}-\mathrm{NMR}\left(\mathrm{CDCl}_{3}, 125 \mathrm{MHz}\right): \delta$ 203.3, 153.7, 141.3, 136.2, 133.04, 128.7 (2C), 128.4 (2C), 110.2, 106.5, 40.0, 31.7, $17.6 \mathrm{ppm}$.

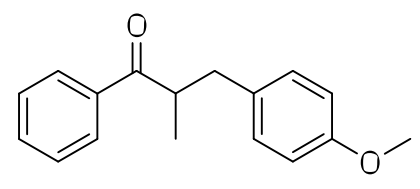

Chemical Formula: $\mathrm{C}_{17} \mathrm{H}_{18} \mathrm{O}_{2}$

\section{- 2-methyl-3-(4-methoxyphenyl)-1-phenylpropan-1-one ${ }^{2}$ (1t)}

According to general procedure $B$, alkylation of acetophenone with (4-methoxyphenyl) methanol (345 mg, 5 equiv) afforded the pure product $1 \mathrm{t}$ as a colorless oil ( $68 \mathrm{mg}, 54 \%$ ) by silica flash column chromatography (pentane/ethyl acetate 95:5). The conversion was determined by ${ }^{1} \mathrm{H}-\mathrm{NMR}$ spectroscopy in the crude product with $11 \%$ of dimethylated derivative observed. ${ }^{1} \mathrm{H}-\mathrm{NMR}\left(\mathbf{C D C l}_{3}, \mathbf{5 0 0} \mathbf{M H z}\right): \delta 7.84(\mathrm{~d}, J=7.0 \mathrm{~Hz}, 2 \mathrm{H}), 7.46(\mathrm{t}, J=7.5$ $\mathrm{Hz}, 1 \mathrm{H}), 7.36(\mathrm{t}, J=7.5 \mathrm{~Hz}, 2 \mathrm{H}), 7.03(\mathrm{~d}, J=8.5 \mathrm{~Hz}, 1 \mathrm{H}), 6.72(\mathrm{~d}, J=8.5 \mathrm{~Hz}, 1 \mathrm{H}), 3.70(\mathrm{~s}$, $3 \mathrm{H}$ ), 3.60 (app sext, $J=7.0 \mathrm{~Hz}, 1 \mathrm{H}$ ), $3.02(\mathrm{dd}, J=14.0 ; 6.4 \mathrm{~Hz}, 1 \mathrm{H}), 2.54$ (dd, $J=14.0 ; 7.9$ $\mathrm{Hz}, 1 \mathrm{H}), 1.11(\mathrm{~d}, J=7.0 \mathrm{~Hz}, 3 \mathrm{H}) \mathrm{ppm} .{ }^{13} \mathrm{C}-\mathrm{NMR}\left(\mathrm{CDCl}_{3}, 125 \mathrm{MHz}\right): \delta 203.9,158.1,136.6$, 133.0, 132.0, 130.1 (2C), 128.7 (2C), 128.3 (2C), $113.8(2 \mathrm{C}), 55.3,43.0,38.6,17.4$ ppm.<smiles>CC(Cc1ccc(I)cc1)C(=O)c1ccccc1</smiles>

Chemical Formula: $\mathrm{C}_{16} \mathrm{H}_{15} \mathrm{IO}$

\section{- 2-methyl-3-(4-iodophenyl)-1-phenylpropan-1-one ${ }^{7}$ (1u)}

According to general procedure $B$, alkylation of acetophenone with (4-iodophenyl)methanol ( $585 \mathrm{mg}, 5$ equiv) afforded the pure product $1 \mathrm{u}$ as a colorless oil ( $98 \mathrm{mg}, 56 \%$ ) by silica flash column chromatography (pentane/ethyl acetate 95:5). The conversion was determined by ${ }^{1} \mathrm{H}-$ NMR spectroscopy in the crude product with $16 \%$ of dimethylated derivative observed. ${ }^{1} \mathrm{H}$ NMR $\left(\mathbf{C D C l}_{3}, \mathbf{5 0 0 ~ M H z}\right): \delta 7.84(\mathrm{~d}, J=7.0 \mathrm{~Hz}, 2 \mathrm{H}), 7.52-7.48(\mathrm{~m}, 3 \mathrm{H}), 7.38(\mathrm{t}, J=7.5 \mathrm{~Hz}$, $2 \mathrm{H}), 6.88(\mathrm{~d}, J=8.0 \mathrm{~Hz}, 2 \mathrm{H}), 3.62(\operatorname{app~sext}, J=7.0 \mathrm{~Hz}, 1 \mathrm{H}), 3.03(\mathrm{dd}, J=14.0 ; 7.0 \mathrm{~Hz}, 1 \mathrm{H})$, 2.57 (dd, $J=14.0 ; 7.5 \mathrm{~Hz}, 1 \mathrm{H}), 1.13(\mathrm{~d}, J=7.0 \mathrm{~Hz}, 3 \mathrm{H}) \mathrm{ppm} .{ }^{13} \mathrm{C}-\mathrm{NMR}\left(\mathrm{CDCl}_{3}, 125 \mathrm{MHz}\right): \delta$

\footnotetext{
${ }^{6}$ Cao, X-N.; Wan, X-M.; Yang. F-L.; Li, K.; Hao, X-Q.; Shao, T; Zhu, X.; Song, M-P. J. Org. Chem , 2018, 83, 3657 - 3668 .

${ }^{7}$ Chun, S.; Chung, Y-K. Org. Lett. 2018, 20, $5583-5586$.
} 
203.3, 139.7, 137.5 (2C), 136.3, 133.1, 131.2 (2C), 128.7 (2C), 128.3 (2C), 91.4, 42.6, 38.8, $17.7 \mathrm{ppm}$.<smiles>CC(Cc1ccc(Br)cc1)C(=O)c1ccccc1</smiles>

Chemical Formula: $\mathrm{C}_{16} \mathrm{H}_{15} \mathrm{BrO}$

\section{- 2-methyl-3-(4-bromophenyl)-1-phenylpropan-1-one ${ }^{1}$ (1v)}

According to general procedure $\mathrm{B}$, alkylation of acetophenone with (4-bromophenyl)methanol ( $465 \mathrm{mg}, 5$ equiv) afforded the pure product $1 \mathrm{v}$ as a colorless oil ( $88 \mathrm{mg}, 58 \%$ ) by silica flash column chromatography (pentane/ethyl acetate 95:5). The conversion was determined by ${ }^{1} \mathrm{H}-$ NMR spectroscopy in the crude product with $18 \%$ of dimethylated derivative observed. ${ }^{1} \mathrm{H}$ NMR $\left(\mathrm{CDCl}_{3}, 500 \mathrm{MHz}\right): \delta 7.90(\mathrm{~d}, J=8.0 \mathrm{~Hz}, 2 \mathrm{H}), 7.59-7.55(\mathrm{~m}, 3 \mathrm{H}), 7.44(\mathrm{t}, J=7.5 \mathrm{~Hz}$, $2 \mathrm{H}), 6.95(\mathrm{~d}, J=8.0 \mathrm{~Hz}, 2 \mathrm{H}), 3.68(\operatorname{app~sext}, J=7.0 \mathrm{~Hz}, 1 \mathrm{H}), 3.10(\mathrm{dd}, J=13.8 ; 7.0 \mathrm{~Hz}, 1 \mathrm{H})$, $2.63(\mathrm{dd}, J=13.8 ; 7.0 \mathrm{~Hz}, 1 \mathrm{H}), 1.20$ (d, $J=7.0 \mathrm{~Hz}, 3 \mathrm{H})$ ppm. ${ }^{13} \mathbf{C}-\mathbf{N M R}\left(\mathbf{C D C l}_{3}, 125 \mathrm{MHz}\right): \delta$ 203.3, 139.7, 137.4 (2C), 136.3, 133.1, 131.2 (2C), 128.7 (2C), 128.3 (2C), 91.4, 42.6, 38.7, $17.7 \mathrm{ppm}$.

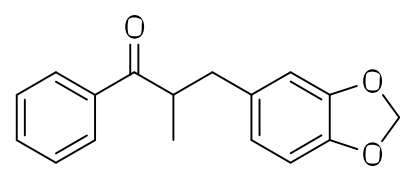

Chemical Formula: $\mathrm{C}_{17} \mathrm{H}_{16} \mathrm{O}_{3}$

\section{- 2-methyl-3-(benzo[d][1,3]dioxol-5-yl)-1-phenylpropan-1-one ${ }^{1}(1 \mathrm{w})$}

According to general procedure $\mathrm{B}$, alkylation of acetophenone with piperonyl alcohol (380 $\mathrm{mg}, 5$ equiv) afforded the pure product $1 \mathrm{w}$ as a colorless oil ( $86 \mathrm{mg}, 64 \%)$ by silica flash column chromatography (pentane/ethyl acetate 95:5). The conversion was determined by ${ }^{1} \mathrm{H}-$ NMR spectroscopy in the crude product with $14 \%$ of dimethylated derivative observed. ${ }^{1} \mathrm{H}$ NMR $\left(\mathrm{CDCl}_{3}, \mathbf{5 0 0} \mathbf{M H z}\right): \delta 7.84(\mathrm{~d}, J=7.5 \mathrm{~Hz}, 2 \mathrm{H}), 7.47(\mathrm{t}, J=7.5 \mathrm{~Hz}, 1 \mathrm{H}), 7.37$ (t, $J=7.5$ $\mathrm{Hz}, 2 \mathrm{H}), 6.64-6.62(\mathrm{~m}, 2 \mathrm{H}), 6.56(\mathrm{dd}, J=13 ; 1.5 \mathrm{~Hz}, 1 \mathrm{H}), 5.83(\mathrm{~m}, 2 \mathrm{H}) 3.59$ (app sext, $J=$ $7.0 \mathrm{~Hz}, 1 \mathrm{H}), 2.99$ (dd, $J=14.0 ; 6.3 \mathrm{~Hz}, 1 \mathrm{H}), 2.52(\mathrm{dd}, J=14.0 ; 7.9 \mathrm{~Hz}, 1 \mathrm{H}), 1.11(\mathrm{~d}, J=7.0$ $\mathrm{Hz}, 3 \mathrm{H}) \mathrm{ppm} .{ }^{13} \mathrm{C}-\mathrm{NMR}\left(\mathrm{CDCl}_{3}, 125 \mathrm{MHz}\right): \delta 203.7,147.6,145.9,136.5,133.7,133.0,128.7$ (2C), $128.3(2 \mathrm{C}), 122.0,109.5,108.2,100.8,43.0,39.1,17.4 \mathrm{ppm}$.

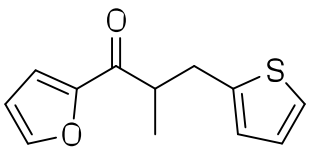

Chemical Formula: $\mathrm{C}_{12} \mathrm{H}_{12} \mathrm{O}_{2} \mathrm{~S}$

\section{- 2-methyl-3-(thiophen-2-yl)-1-(furan-2-yl)propan-1-one (1x)}

According to general procedure $\mathrm{C}$, alkylation of 2-acetylfuran with thiophen-2-ylmethanol $(0.237 \mathrm{~mL}, 5$ equiv) afforded the pure product $1 \mathrm{x}$ as a yellow oil $(98 \mathrm{mg}, 89 \%)$ by silica flash column chromatography (pentane/ethyl acetate 98:2). ${ }^{1} \mathbf{H}-\mathrm{NMR}\left(\mathbf{C D C l}_{\mathbf{3}}, \mathbf{5 0 0} \mathbf{~ M H z}\right): \delta 7.56$ $(\mathrm{dd}, J=1.5 ; 1 \mathrm{~Hz}, 1 \mathrm{H}), 7.16(\mathrm{dd}, J=3.5 ; 1.0 \mathrm{~Hz}, 1 \mathrm{H}), 7.07(\mathrm{dd}, J=5.0 ; 1.5 \mathrm{~Hz}, 1 \mathrm{H}), 6.85(\mathrm{dd}$, $J=5.0 ; 3.5 \mathrm{~Hz}, 1 \mathrm{H}), 6.77(\mathrm{dd}, J=3.0 ; 1.5 \mathrm{~Hz}, 1 \mathrm{H}), 6.49(\mathrm{dd}, J=3.5 ; 2.0 \mathrm{~Hz}, 1 \mathrm{H}), 3.49$ (app sext, $J=7.0 \mathrm{~Hz}, 1 \mathrm{H}$ ), 3.30 (dd, $J=14.7 ; 7.0 \mathrm{~Hz}, 1 \mathrm{H}), 2.89$ (dd, $J=14.7 ; 7.0 \mathrm{~Hz}, 1 \mathrm{H}), 1.22(\mathrm{~d}$, $J=7.0 \mathrm{~Hz}, 3 \mathrm{H}) \mathrm{ppm} .{ }^{13} \mathbf{C}-\mathrm{NMR}\left(\mathrm{CDCl}_{3}, \mathbf{1 2 5}_{\mathbf{M H z}}\right): \delta 192.1,152.3,146.6,142.2,126.8,125.7$, 123.7, 117.6, 112.3, 44.1, 33.0, 17.4 ppm. IR (neat): v 3131, 2971, 2932, 1668, 1566, 1465, 
1395, 1263, 1156, 1082, 1014, 883, 760, 694, 593, $507 \mathrm{~cm}^{-1}$. HRMS [M+H] : calculated $\mathrm{C}_{15} \mathrm{H}_{13} \mathrm{O}_{2} \mathrm{~S} 221.0636$; found: 221.0634 .

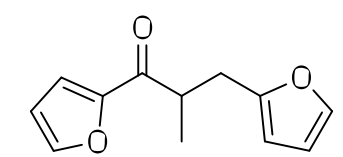

Chemical Formula: $\mathrm{C}_{12} \mathrm{H}_{12} \mathrm{O}_{3}$

\section{- 1,3-di(furan-2-yl)-2-methylpropan-1-one (1y)}

According to general procedure $\mathrm{C}$, alkylation of 2-acetylfuran with furan-2-ylmethanol $(0.217$ $\mathrm{mL}, 5$ equiv) afforded the pure product $1 \mathrm{y}$ as a yellow oil $(84 \mathrm{mg}, 82 \%)$ by silica flash column chromatography (pentane/ethyl acetate 98:2). ${ }^{1} \mathbf{H}-\mathbf{N M R}\left(\mathbf{C D C l}_{3}, \mathbf{5 0 0} \mathbf{~ M H z}\right): \delta 7.52$ (dd, $J=$ 2.0; $1.0 \mathrm{~Hz}, 1 \mathrm{H}), 7.21(\mathrm{dd}, J=2.0 ; 1.0 \mathrm{~Hz}, 1 \mathrm{H}), 7.19(\mathrm{dd}, J=3.5 ; 0.5 \mathrm{~Hz}, 1 \mathrm{H}), 6.51(\mathrm{dd}, J=$ 3.5; $2.0 \mathrm{~Hz}, 1 \mathrm{H}$ ), 6.23 (dd, $J=3.5 ; 2.0 \mathrm{~Hz}, 1 \mathrm{H}), 6.00$ (dd, J=3.0; $0.5 \mathrm{~Hz}, 1 \mathrm{H}$ ), 3.58 (app sext, $J=7.0 \mathrm{~Hz}, 1 \mathrm{H}), 3.08(\mathrm{dd}, J=15.0 ; 6.4 \mathrm{~Hz}, 1 \mathrm{H}), 2.74(\mathrm{dd}, J=15 ; 7.9 \mathrm{~Hz}, 1 \mathrm{H}), 1.21(\mathrm{~d}, J=7.0$ $\mathrm{Hz}, 3 \mathrm{H})$ ppm. ${ }^{13} \mathrm{C}-\mathrm{NMR}\left(\mathrm{CDCl}_{3}, \mathbf{1 2 5} \mathbf{M H z}\right): \delta$ 192.1, 153.4, 152.1, 146.6, 141.3, 117.6, 112.2, 110.2, 106.6, 40.9, 31.4, 17.2 ppm. IR (neat): v 3421, 2972, 2932, 1715, 1667, 1566, 1496, 1466, 1397, 1262, 1083, 1016, 882, 764, 593, $498 \mathrm{~cm}^{-1}$. HRMS [M+Na] : calculated $\mathrm{C}_{12} \mathrm{H}_{12} \mathrm{O}_{3} \mathrm{Na} 227.0684$; found: 227.0686 .

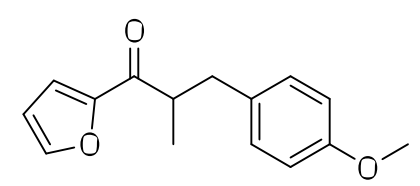

Chemical Formula: $\mathrm{C}_{15} \mathrm{H}_{16} \mathrm{O}_{3}$

\section{- 2-methyl-3-(4-methoxyphenyl)-1-(furan-2-yl)propan-1-one (1z)}

According to general procedure $C$, alkylation of 2-acetylfuran with (4methoxyphenyl)methanol ( $345 \mathrm{mg}, 5$ equiv) afforded the pure product $1 \mathrm{z}$ as a yellow oil (71 $\mathrm{mg}, 58 \%$ ) by silica flash column chromatography (pentane/ethyl acetate 90:10). The conversion was determined by ${ }^{1} \mathrm{H}-\mathrm{NMR}$ spectroscopy in the crude product with $13 \%$ of dimethylated derivative observed. ${ }^{1} \mathrm{H}-\mathrm{NMR}\left(\mathrm{CDCl}_{3}, \mathbf{5 0 0} \mathbf{~ M H z}\right): \delta 7.57(\mathrm{~s}, 1 \mathrm{H}), 7.14(\mathrm{~d}, \mathrm{~J}=3.5$ $\mathrm{Hz}, 1 \mathrm{H}), 7.10(\mathrm{~d}, J=8 \mathrm{~Hz}, 2 \mathrm{H}), 6.79(\mathrm{~d}, J=8.5 \mathrm{~Hz}, 2 \mathrm{H}), 6.50(\mathrm{~m}, 1 \mathrm{H}), 3.77(\mathrm{~s}, 3 \mathrm{H}), 3.44$ (app sext, $J=7.0 \mathrm{~Hz}, 1 \mathrm{H}$ ), $3.06(\mathrm{dd}, J=13.7 ; 6.3 \mathrm{~Hz}, 1 \mathrm{H}), 2.61$ (dd, $J=13.7 ; 7.9 \mathrm{~Hz}, 1 \mathrm{H}), 1.18$ (d, $J=7.0 \mathrm{~Hz}, 3 \mathrm{H}) \mathrm{ppm} .{ }^{13} \mathrm{C}-\mathrm{NMR}\left(\mathrm{CDCl}_{3}, 125 \mathrm{MHz}\right): \delta 192.9,158.0,152.4,146.4,131.8,130.1$ (2C), 117.4, 113.8 (2C), 112.2, 55.3, 43.9, 38.4, 16.9 ppm. IR (neat): v 3128, 2970, 2933, 2835, 1669, 1611, 1584, 1512, 1465, 1395, 1244, 1177, 1083, 1031, 814, 761, 594, $523 \mathrm{~cm}^{-}$ ${ }^{1}$. HRMS [M+Na] $]^{+}$: calculated $\mathrm{C}_{15} \mathrm{H}_{16} \mathrm{O}_{3} \mathrm{Na} 267.0997$; found: 267.1001 .

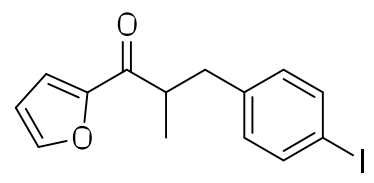

Chemical Formula: $\mathrm{C}_{14} \mathrm{H}_{13} \mathrm{IO}_{2}$

\section{- 2-methyl-3-(4-iodophenyl)-1-phenylpropan-1-one (1aa)}

According to general procedure $\mathrm{C}$, alkylation of 2-acetylfuran with (4-iodophenyl)methanol (585 mg, 5 equiv) afforded the pure product 1aa as a light yellow oil ( $84 \mathrm{mg}, 50 \%$ ) by silica flash column chromatography (pentane/ethyl acetate 90:10). The conversion was determined by ${ }^{1} \mathrm{H}$-NMR spectroscopy in the crude product with $16 \%$ of dimethylated derivative observed. ${ }^{1} \mathrm{H}-\mathrm{NMR}\left(\mathrm{CDCl}_{3}, \mathbf{5 0 0} \mathrm{MHz}\right): \delta$ 7.54-7.52 (m, 3H), $7.10(\mathrm{dd}, J=3.5 ; 1.0 \mathrm{~Hz}, 1 \mathrm{H}), 6.90(\mathrm{~d}, J=$ 
8.0 Hz, 2H), 6.47 (dd, $J=3.5 ; 2.0 \mathrm{~Hz}, 1 \mathrm{H}$ ), 3.41 (app sext, $J=7.0 \mathrm{~Hz}, 1 \mathrm{H}), 3.02$ (dd, $J=$ 13.7; 7.0 Hz, 1H), 2.56 (dd, $J=13.7 ; 7.0 \mathrm{~Hz}, 1 \mathrm{H}), 1.15$ (d, $J=7.0 \mathrm{~Hz}, 3 \mathrm{H}$ ) ppm. ${ }^{13} \mathrm{C}-\mathrm{NMR}$ $\left(\mathrm{CDCl}_{3}, 125 \mathrm{MHz}\right): \delta 192.3,152.3,146.5,139.4,137.4$ (2C), $131.2(2 \mathrm{C}), 117.5,112.3,91.5$, 43.5, 38.5, 17.2 ppm. IR (neat): v 2971, 2925, 1669, 1565, 1465, 1394, 1254, 1154, 1081, 1006, 883, 793, 758, 592, 515, $486 \mathrm{~cm}^{-1}$. HRMS [M+H] $]^{+}$: calculated $\mathrm{C}_{14} \mathrm{H}_{14} \mathrm{IO}_{2} 341.0038$; found: 341.0036 .

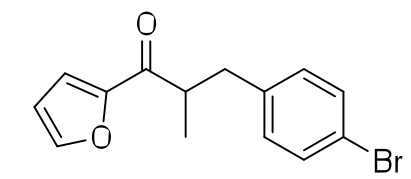

Chemical Formula: $\mathrm{C}_{14} \mathrm{H}_{13} \mathrm{BrO}_{2}$

\section{- 2-methyl-3-(4-bromophenyl)-1-phenylpropan-1-one (1ab)}

According to general procedure $\mathrm{C}$, alkylation of 2-acetylfuran with (4-bromophenyl)methanol (465 mg, 5 equiv) afforded the pure product 1ab as a light yellow oil (76 mg, $52 \%$ ) by silica flash column chromatography (pentane/ethyl acetate 90:10). The conversion was determined by ${ }^{1} \mathrm{H}$-NMR spectroscopy in the crude product with $19 \%$ of dimethylated derivative observed. ${ }^{1} \mathrm{H}-\mathrm{NMR}\left(\mathrm{CDCl}_{3}, \mathbf{5 0 0} \mathrm{MHz}\right): \delta 7.56(\mathrm{dd}, J=1.5 ; 0.5 \mathrm{~Hz}, 1 \mathrm{H}), 7.35(\mathrm{~d}, J=8.5 \mathrm{~Hz}, 2 \mathrm{H}), 7.13$ (d, $J=4.0 ; 1.5 \mathrm{~Hz}, 1 \mathrm{H}), 7.05(\mathrm{~d}, J=8.5 \mathrm{~Hz}, 2 \mathrm{H}), 6.50(\mathrm{dd}, J=4.0 ; 1.5 \mathrm{~Hz}, 1 \mathrm{H}), 3.45$ (app sext, $J$ $=7.0 \mathrm{~Hz}, 1 \mathrm{H}), 3.06(\mathrm{dd}, J=13.7 ; 7.0 \mathrm{~Hz}, 1 \mathrm{H}), 2.61(\mathrm{dd}, J=13.7 ; 7.4 \mathrm{~Hz}, 1 \mathrm{H}), 1.18(\mathrm{~d}, J=7.0$ $\mathrm{Hz}, 3 \mathrm{H}) \mathrm{ppm} .{ }^{13} \mathrm{C}-\mathrm{NMR}\left(\mathrm{CDCl}_{3}, 125 \mathrm{MHz}\right): \delta$ 192.3, 152.3, 146.5, 138.8, $131.4(2 \mathrm{C}), 130.9$ (2C), 120.1, 117.5, 112.3, 43.5, 38.4, 17.2 ppm. IR (neat): v 2972, 2901, 1671, 1566, 1488, $1465,1394,1251,1067,1057,882,759,593 \mathrm{~cm}^{-1}$. HRMS [M+H] : calculated $\mathrm{C}_{14} \mathrm{H}_{14} \mathrm{BrO}_{2}$ 293.0177; found: 293.0179 .

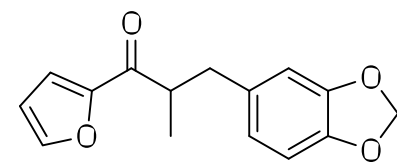

Chemical Formula: $\mathrm{C}_{15} \mathrm{H}_{14} \mathrm{O}_{4}$

\section{- 2-methyl-3-(benzo[d][1,3]dioxol-5-yl)-1-(furan-2-yl)propan-1-one (1ac)}

According to general procedure C, alkylation of 2-acetylfuran with piperonyl alcohol $(380 \mathrm{mg}$, 5 equiv) afforded the pure product $1 \mathrm{ac}$ as a colorless oil $(67 \mathrm{mg}, 52 \%)$ by silica flash column chromatography (pentane/ethyl acetate 90:10). The conversion was determined by ${ }^{1} \mathrm{H}-\mathrm{NMR}$ spectroscopy in the crude product with $11 \%$ of dimethylated derivative observed. ${ }^{1} \mathbf{H}-\mathbf{N M R}$ $\left(\mathrm{CDCl}_{3}, 500 \mathrm{MHz}\right): \delta 7.55$ (dd, $\left.J=2.0 ; 1.0 \mathrm{~Hz}, 1 \mathrm{H}\right), 7.13$ (dd, $\left.J=3.5 ; 0.5 \mathrm{~Hz}, 1 \mathrm{H}\right), 6.69-6.67$ $(\mathrm{m}, 2 \mathrm{H}), 6.61(\mathrm{~d}, J=7.5 ; 2.0 \mathrm{~Hz}, 1 \mathrm{H}), 6.49(\mathrm{dd}, J=3.5 ; 1.5 \mathrm{~Hz}, 1 \mathrm{H}), 3.41$ (app sext, $J=7.0$ $\mathrm{Hz}, 1 \mathrm{H}), 3.01(\mathrm{dd}, J=13.7 ; 7.0 \mathrm{~Hz}, 1 \mathrm{H}), 2.56(\mathrm{dd}, J=13.7 ; 7.5 \mathrm{~Hz}, 1 \mathrm{H}), 1.16(\mathrm{~d}, J=7.0 \mathrm{~Hz}$, 3H) ppm. ${ }^{13} \mathbf{C}-N M R\left(\mathbf{C D C l}_{3}, 125 \mathrm{MHz}\right): \delta$ 192.6, 152.4, 147.5, 146.4, 145.9, 133.5, 122.1, 117.4, 112.3, 109.5, 108.2, 100.8, 43.9, 38.9, 16.9 ppm. IR (neat): v 2972, 2901, 1668, 1566 , 1488, 1466, 1394, 1243, 1079, 1066, 1038, 928, 808, 761, 594, $494 \mathrm{~cm}^{-1}$. HRMS [M+H] : calculated $\mathrm{C}_{15} \mathrm{H}_{14} \mathrm{O}_{4}$ 259.0892; found: 258.0891 . 
<smiles>CC(Cc1cccs1)C(=O)C1CC1</smiles>

Chemical Formula: $\mathrm{C}_{11} \mathrm{H}_{14} \mathrm{OS}$

\section{- 1-cyclopropyl-2-methyl-3-(thiophen-2-yl)propan-1-one (1ad)}

In a $15 \mathrm{~mL}$ flame-dried Schlenk tube equipped with a stirring bar, 1-cyclopropylethan-1-one (0.5 mmol, $47 \mu \mathrm{L})$, thiophen-2-ylmethanol (0.237 mL, 5 equiv), $\mathrm{KOH}(2.8 \mathrm{mg}, 10 \mathrm{~mol} \%$ ), methanol (25 equiv, $0.5 \mathrm{~mL}$ ), iron complex Fe2 (4.56 mg, $2 \mathrm{~mol} \%$ ) and $\mathrm{K}_{3} \mathrm{PO}_{4}(212 \mathrm{mg}, 2$ equiv) were poured in under an argon atmosphere. The mixture was rapidly stirred at room temperature for 2 min and then placed into a pre-heated oil bath at $90^{\circ} \mathrm{C}$ and stirred over 24 hours. The mixture was cooled-down to room temperature, filtrated over silica with diethyl ether and concentrated under reduced pressure. The conversion was determined by ${ }^{1} \mathrm{H}-\mathrm{NMR}$ spectroscopy with $46 \%$ of monobenzylated derivative observed. Then the residue was purified by flash chromatography on silica gel using pentane-ethyl acetate (98:2) as eluent to afford the pure product $1 \mathrm{ad}$ as a colorless oil $(48 \mathrm{mg}, 49 \%) .{ }^{1} \mathrm{H}-\mathrm{NMR}\left(\mathrm{CDCl}_{3}, \mathbf{5 0 0} \mathbf{M H z}\right): \delta$ $7.12(\mathrm{dd}, J=5.1 ; 1.2 \mathrm{~Hz}, 1 \mathrm{H}), \delta 6.90(\mathrm{dd}, J=5.1 ; 3.4 \mathrm{~Hz}, 1 \mathrm{H}), \delta 6.78(\mathrm{app} \mathrm{dd}, J=3.4 ; 1.1$ $\mathrm{Hz}, 1 \mathrm{H}), 3.23$ (dd, $J=14.8 ; 6.8 \mathrm{~Hz}, 1 \mathrm{H}), 2.97$ (app sext, $J=7.0 \mathrm{~Hz}, 1 \mathrm{H}), 2.82(\mathrm{dd}, J=14.8$; $6.9 \mathrm{~Hz}, 1 \mathrm{H}), 1.97-1.93(\mathrm{~m}, 1 \mathrm{H}), 1.18(\mathrm{~d}, J=7.0 \mathrm{~Hz}, 3 \mathrm{H}), 1.01-0.97(\mathrm{~m}, 2 \mathrm{H}), 0.88-0.83(\mathrm{~m}, 2 \mathrm{H})$ ppm. ${ }^{13} \mathrm{C}-\mathrm{NMR}\left(\mathrm{CDCl}_{3}, 125 \mathrm{MHz}\right): \delta 213.3,142.4,126.8,125.5,123.6,49.3,32.8,19.7,16.6$, 11.1, 11.0 ppm. IR (neat): v 1668, 1592, 1457, 1394, 1249, 1182, 1066, 1063, 932, 826, 761, $487 \mathrm{~cm}^{-1}$. HRMS [M+H] $]^{+}$: calculated $\mathrm{C}_{11} \mathrm{H}_{14} \mathrm{OS}$ 195.0844; found: 195.0844 .<smiles>COC(CN(C)Cc1ccc(C(=O)C(C)Cc2ccccc2)cc1)OC</smiles>

- 1-(4-(((2,2-dimethoxyethyl)(methyl)amino)methyl)phenyl)-2-methyl-3-phenylpropan1-one (2a)

According to general procedure A, alkylation of 1-(4-\{[(2,2-dimethoxyethyl) (methyl)amino]methyl\}phenyl)ethan-1-one ${ }^{1}(0.5 \mathrm{mmol}, 126 \mathrm{mg})$ afforded the pure product $2 \mathrm{a}$ as a colorless oil (126 mg, $71 \%$ ) by silica flash column chromatography (pentane/diethyl ether 50:50). ${ }^{1} \mathrm{H}-\mathrm{NMR}\left(\mathrm{CDCl}_{3}, \mathbf{5 0 0} \mathbf{~ M H z}\right): \delta 7.87(\mathrm{~d}, \mathrm{~J}=8.3 \mathrm{~Hz}, 2 \mathrm{H}), 7.41(\mathrm{~d}, J=8.3 \mathrm{~Hz}, 2 \mathrm{H})$, 7.27-7.24 (m, 2H), 7.20-7.16 (m, 3H), $4.51(\mathrm{t}, J=5.3 \mathrm{~Hz}, 1 \mathrm{H}), 3.69($ app sext, $J=7.1 \mathrm{~Hz}$, $1 \mathrm{H}), 3.61(\mathrm{~s}, 2 \mathrm{H}), 3.33(\mathrm{~s}, 6 \mathrm{H}), 3.14(\mathrm{dd}, J=13.7 ; 6.4 \mathrm{~Hz}, 1 \mathrm{H}), 2.66(\mathrm{dd}, J=13.7 ; 7.4 \mathrm{~Hz}$, 1H), $2.55(\mathrm{~d}, J=5.3 \mathrm{~Hz}, 2 \mathrm{H}), 2.29(\mathrm{~s}, 3 \mathrm{H}), 1.18(\mathrm{~d}, J=6.9 \mathrm{~Hz}, 3 \mathrm{H})$ ppm. ${ }^{13} \mathbf{C}-\mathbf{N M R}\left(\mathbf{C D C l}_{3}\right.$, $125 \mathrm{MHz}$ ): $\delta$ 203.5, 144.5, 140.0, 135.4, 129.2 (2C), 129.1 (2C), 128.4 (2C), 126.2, 102.9, 62.5, 58.5, 53.3 (2C), 43.8, 42.8, 39.4, 19.2, 17.5 ppm. IR (neat): v 3027, 2932, 2829, 1678, 1606, 1571, 1496, 1453, 1363, 1229, 1123, 1072, 972, 875, 746, 699, $518 \mathrm{~cm}^{-1}$. HRMS $[\mathrm{M}+\mathrm{H}]^{+}$: calculated $\mathrm{C}_{22} \mathrm{H}_{29} \mathrm{NO}_{3}$ 356.2226; found: 356.2225 . 
<smiles>COC(CN(C)Cc1ccc(C(=O)C(C)Cc2ccco2)cc1)OC</smiles>

Chemical Formula: $\mathrm{C}_{20} \mathrm{H}_{27} \mathrm{NO}_{4}$

- 1-(4-((2,2-dimethoxyethyl)(methyl)amino)methyl)phenyl)-3-(furan-2-yl)-2methylpropan-1-one (2b)

According to general procedure $D$, alkylation of 1-(4-\{[(2,2-dimethoxyethyl) (methyl)amino]methyl\}phenyl)ethan-1-one ${ }^{1}$ with furan-2-ylmethanol $(0.217 \mathrm{~mL}, 5$ equiv) afforded the pure product $\mathbf{2 b}$ as a light yellow oil $(126 \mathrm{mg}, 73 \%)$ by silica flash column chromatography (pentane/diethyl ether 40:60). ${ }^{1} \mathrm{H}-\mathrm{NMR}\left(\mathrm{CDCl}_{3}, \mathbf{5 0 0} \mathbf{~ M H z}\right): \delta 7.91$ (d, $J=8.3$ $\mathrm{Hz}, 2 \mathrm{H}), 7.43(\mathrm{~d}, J=8.3 \mathrm{~Hz}, 2 \mathrm{H}), 7.28(\mathrm{~d}, J=1.1 \mathrm{~Hz}, 1 \mathrm{H}), 6.24-6.23(\mathrm{~m}, 1 \mathrm{H}), 6.00(\mathrm{~d}, J=1.9$ $\mathrm{Hz}, 1 \mathrm{H}), 4.51(\mathrm{t}, J=5.3 \mathrm{~Hz}, 1 \mathrm{H}), 3.81(\operatorname{app} s e x t, J=7.1 \mathrm{~Hz}, 1 \mathrm{H}), 3.62(\mathrm{~s}, 2 \mathrm{H}), 3.33(\mathrm{~s}, 6 \mathrm{H})$, $3.12(\mathrm{dd}, J=15.0 ; 6.8 \mathrm{~Hz}, 1 \mathrm{H}), 2.73(\mathrm{dd}, J=15.0 ; 7.6 \mathrm{~Hz}, 1 \mathrm{H}), 2.55(\mathrm{~d}, J=5.3 \mathrm{~Hz}, 2 \mathrm{H}$ ), 2.29 $(\mathrm{s}, 3 \mathrm{H}), 1.20(\mathrm{~d}, J=7.0 \mathrm{~Hz}, 3 \mathrm{H})$ ppm. ${ }^{13} \mathrm{C}-\mathrm{NMR}\left(\mathrm{CDCl}_{3}, 125 \mathrm{MHz}\right): \delta$ 203.0, 153.7, 144.6, 141.2, 135.1, 129.2 (2C), 128.4 (2C), 110.2, 106.5, 102.9, 62.5, 58.5, 53.3 (2C), 43.3, 40.0, 31.7, 17.6 ppm. IR (neat): v 2934, 2830, 1679, 1606, 1566, 1455, 1362, 1224, 1123, 1071, 974, 875, 730, $599 \mathrm{~cm}^{-1}$. HRMS [M+H] $]^{+}$calculated $\mathrm{C}_{20} \mathrm{H}_{27} \mathrm{NO}_{4} 346.2018$; found: 346.2014.

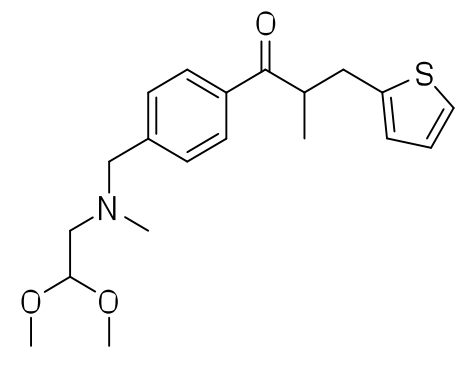

Chemical Formula: $\mathrm{C}_{20} \mathrm{H}_{27} \mathrm{NO}_{3} \mathrm{~S}$

- 1-(4-(((2,2-dimethoxyethyl)(methyl)amino)methyl)phenyl)-2-methyl-3-(thiophen-2yl)propan-1-one (2c)

According to general procedure $D$, alkylation of 1-(4-\{[(2,2-dimethoxyethyl) (methyl)amino]methyl\}phenyl)ethan-1-one ${ }^{1}$ with thiophen-2-ylmethanol (0.237 mL, 5 equiv) afforded the pure product $\mathbf{2 c}$ as a colorless oil $(129 \mathrm{mg}, 71 \%)$ by silica flash column chromatography (pentane/diethyl ether 40:60). ${ }^{1} \mathbf{H}-\mathbf{N M R}\left(\mathrm{CDCl}_{3}, \mathbf{5 0 0} \mathbf{~ M H z}\right): \delta 7.89$ (d, J = 8.3 $\mathrm{Hz}, 2 \mathrm{H}), 7.42(\mathrm{~d}, J=8.3 \mathrm{~Hz}, 2 \mathrm{H}), 7.09(\mathrm{dd}, J=5.1 ; 1.1 \mathrm{~Hz}, 1 \mathrm{H}), 6.87(\mathrm{dd}, J=5.1 ; 3.4 \mathrm{~Hz}$, $1 \mathrm{H}), 6.79(\mathrm{dd}, J=3.4 ; 0.9 \mathrm{~Hz}, 1 \mathrm{H}), 4.51(\mathrm{t}, J=5.3 \mathrm{~Hz}, 1 \mathrm{H}), 3.72(\operatorname{app} \operatorname{sext}, J=7.0 \mathrm{~Hz}, 1 \mathrm{H})$, 3.62 (s, 2H), 3.35 (app dd, $J=14.8 ; 6.4 \mathrm{~Hz}, 1 \mathrm{H}), 3.33(\mathrm{~s}, 6 \mathrm{H}), 2.92(\mathrm{dd}, J=14.8 ; 7.2 \mathrm{~Hz}, 1 \mathrm{H}$ ), $2.55(\mathrm{~d}, J=5.3 \mathrm{~Hz}, 2 \mathrm{H}), 2.29(\mathrm{~s}, 3 \mathrm{H}), 1.24(\mathrm{~d}, J=7.0 \mathrm{~Hz}, 3 \mathrm{H})$ ppm. ${ }^{13} \mathbf{C}-\mathbf{N M R}\left(\mathrm{CDCl}_{3}, 125\right.$ MHz): $\delta 203.0,142.5,129.2,128.4(2 \mathrm{C}), 126.8,125.6,123.6,102.9,62.5,58.5,53.4(2 \mathrm{C})$, 43.3, 33.3, 17.9 ppm. IR (neat): v 2932, 2829, 1678, 1606, 1571, 1454, 1283, 1225, 1123, 1068, 972, 694, $482 \mathrm{~cm}^{-1}$. HRMS [M+H] $]^{+}$calculated $\mathrm{C}_{20} \mathrm{H}_{27} \mathrm{NO}_{3} \mathrm{~S} 362.1790$; found: 362.1790 . 


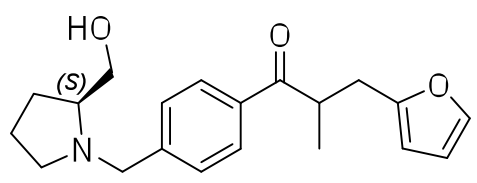

Chemical Formula: $\mathrm{C}_{20} \mathrm{H}_{25} \mathrm{NO}_{3}$

\section{- 3-(furan-2-yl)-1-(4-(((S)-2-(hydroxymethyl)pyrrolidin-1-yl)methyl)phenyl)-2-} methylpropan-1-one (2d)

In a $15 \mathrm{~mL}$ flame-dried Schlenk tube equipped with a stirring bar, 1-(4-\{[(2S)-2-(hydroxymethyl)pyrrolidin-1-yl]methyl\}phenyl)ethan-1-one ${ }^{1}(0.5 \mathrm{mmol}, 117 \mathrm{mg})$, furan-2-ylmethanol $(0.217 \mathrm{~mL}, 5$ equiv), $\mathrm{KOH}(2.8 \mathrm{mg}, 10 \mathrm{~mol} \%)$, methanol (25 equiv, 0.5 $\mathrm{mL}$ ), iron complex Fe2 (4.56 mg, $2 \mathrm{~mol} \%$ ) and $\mathrm{K}_{3} \mathrm{PO}_{4}(212 \mathrm{mg}, 2$ equiv) were poured in under an argon atmosphere. The mixture was rapidly stirred at room temperature for 2 min and then placed into a pre-heated oil bath at $90^{\circ} \mathrm{C}$ and stirred over 24 hours. The mixture was cooled-down to room temperature, filtrated over silica with diethyl ether and concentrated under reduced pressure. The conversion was determined by ${ }^{1} \mathrm{H}-\mathrm{NMR}$ spectroscopy, and then, the residue was purified by flash chromatography on silica gel using dichloromethane-methanol (98:2) as eluent to afford the pure product $\mathbf{2} \mathbf{d}$ as a mix of two diastereomers as a yellow oil (69 mg, $42 \%)$. Major diastereomer: ${ }^{1} \mathbf{H}-\mathbf{N M R}\left(\mathbf{C D C l}_{\mathbf{3}}, \mathbf{5 0 0}\right.$ MHz): $\delta 7.92(\mathrm{~d}, J=8.0 \mathrm{~Hz}, 2 \mathrm{H}), 7.47(\mathrm{~d}, J=7.8 \mathrm{~Hz}, 2 \mathrm{H}), 7.28-7.27(\mathrm{~m}, 1 \mathrm{H}), 6.22(\operatorname{app~dd}, J$ $=3.1 ; 1.9 \mathrm{~Hz}, 1 \mathrm{H}), 6.00(\mathrm{~d}, J=3.1 \mathrm{~Hz}, 1 \mathrm{H}), 4.11(\mathrm{~d}, J=12.5 \mathrm{~Hz}, 1 \mathrm{H}), 3.80$ (app sext, $J=7.0$ $\mathrm{Hz}, 1 \mathrm{H}), 3.67$ (app dd, $J=11.3 ; 3.4 \mathrm{~Hz}, 1 \mathrm{H}), 3.57-3.52(\mathrm{~m}, 2 \mathrm{H}), 3.15-3.07(\mathrm{~m}, 1 \mathrm{H}), 2.73$ (app dd, $J=15.1 ; 7.4 \mathrm{~Hz}, 1 \mathrm{H}), 1.99-1.79(\mathrm{~m}, 5 \mathrm{H}), 1.21(\mathrm{~d}, J=6.9 \mathrm{~Hz}, 3 \mathrm{H})$ ppm. ${ }^{13} \mathbf{C}-\mathbf{N M R}\left(\mathbf{C D C l}_{3}\right.$, $125 \mathrm{MHz}$ ): $\delta$ 202.8, 153.6, 141.3, 128.7, 110.3, 106.5, 105.4, 61.7, 58.5, 54.5, 40.1, 31.7, 23.5, 17.6 ppm. HRMS [M+H] $]^{+}$: calculated $\mathrm{C}_{20} \mathrm{H}_{25} \mathrm{NO}_{3}$ 328.1913; found: 328.1916. 
Part 4: Mechanistic studies

Scheme S1: Kinetic of the differents steps of the reaction

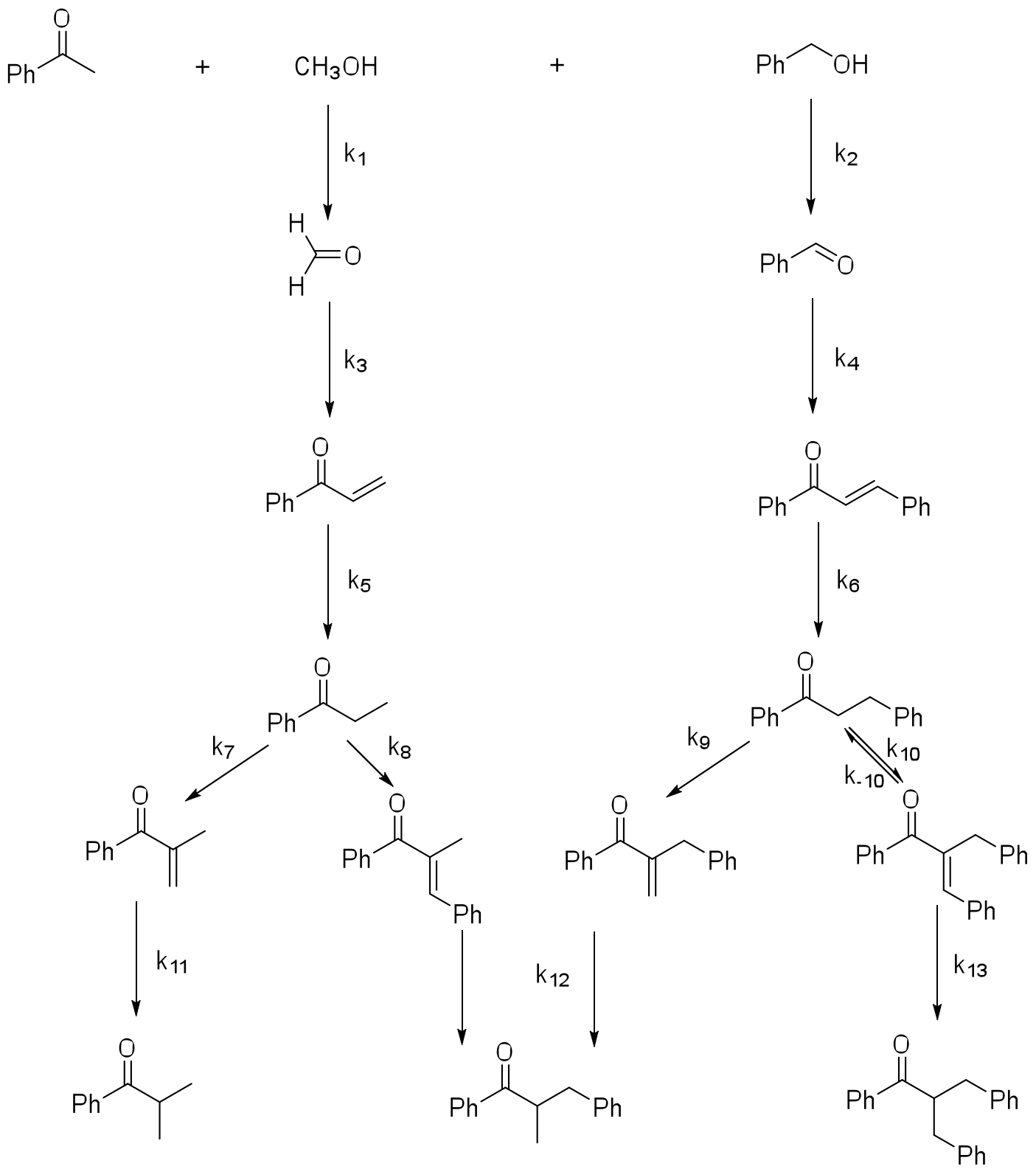




\section{Scheme S2: Cross-over reaction}
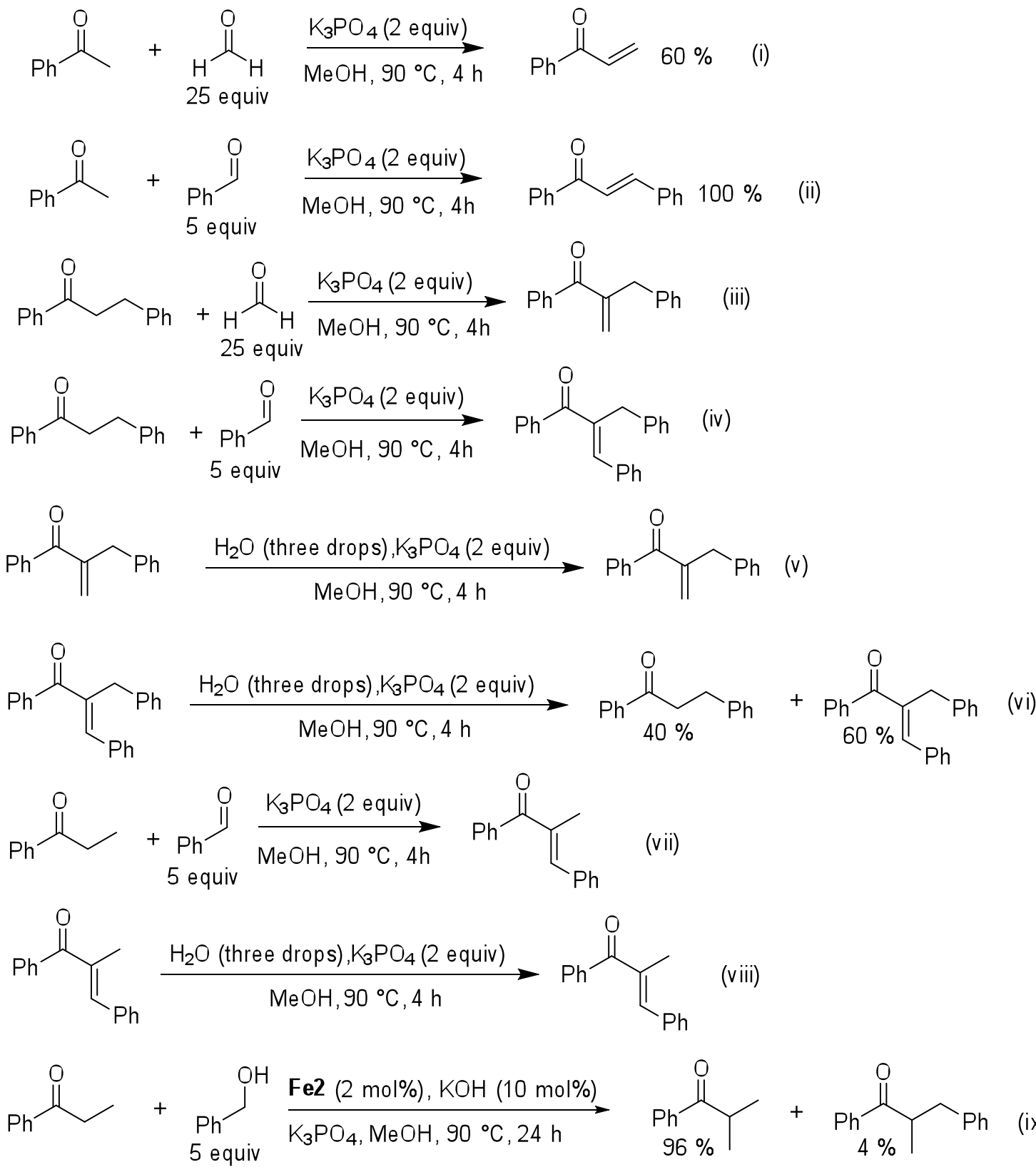


\section{Scheme S3: Deuterium-labeling experiment}
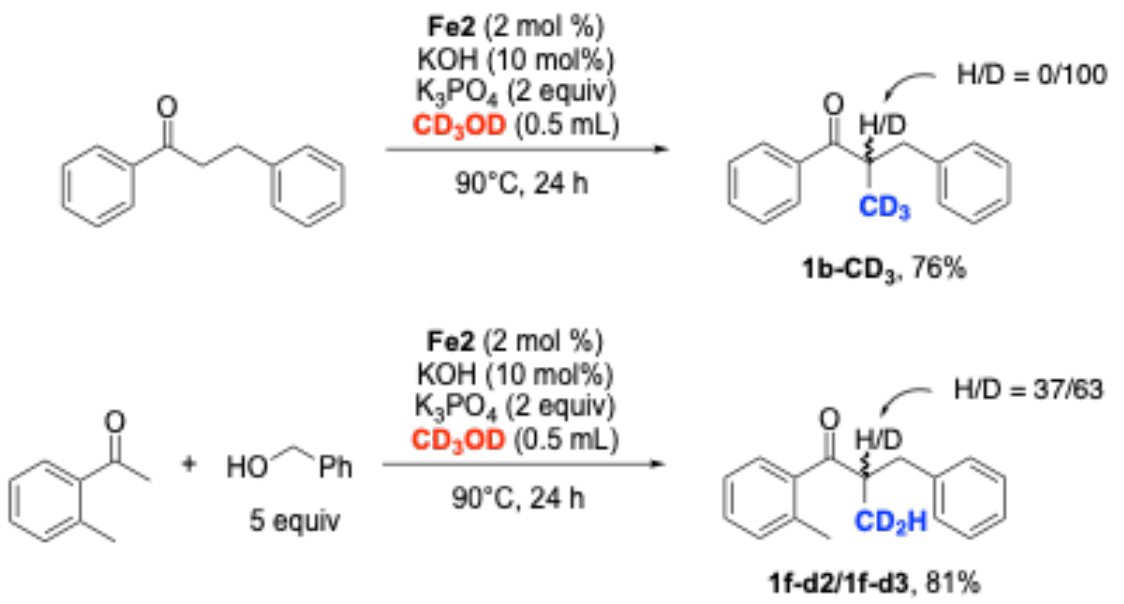<smiles>[2H]C(C)(Cc1ccccc1)C(=O)c1ccccc1</smiles>

Chemical Formula: $\mathrm{C}_{16} \mathrm{H}_{12} \mathrm{D}_{4} \mathrm{O}$

\section{- 2-methyl-1,3-diphenylpropan-1-one-2,3,3,3- $d_{4}{ }^{8}\left(1 b-d_{4}\right)$}

In a $15 \mathrm{~mL}$ flame-dried Schlenk tube equipped with a stirring bar, 1,3-Diphenyl-1-propanone ${ }^{9}$ ( $0.5 \mathrm{mmol}, 102.5 \mathrm{mg}, 1$ equiv), $\mathrm{KOH}\left(2.8 \mathrm{mg}, 10 \mathrm{~mol} \%\right.$ ), methanol- $d_{4}$ ( 25 equiv, $0.5 \mathrm{~mL}$ ), iron complex Fe2 (4.56 mg, 2 mol \%) and $\mathrm{K}_{3} \mathrm{PO}_{4}(212 \mathrm{mg}, 2$ equiv) were poured in under an argon atmosphere. The mixture was rapidly stirred at room temperature for 2 min and then placed into a pre-heated oil bath at $90^{\circ} \mathrm{C}$ and stirred over 24 hours. The mixture was cooleddown to room temperature, filtrated over silica with diethyl ether and concentrated under reduced pressure. The conversion was determined by ${ }^{1} \mathrm{H}-\mathrm{NMR}$ spectroscopy, and then, the residue was purified by flash chromatography on silica gel using pentane-ethyl acetate (95:5) as eluent to afford the pure product $\mathbf{1 b}-\boldsymbol{d}_{\mathbf{4}}$ as a colorless oil $(87 \mathrm{mg}, 76 \%)$. ${ }^{1} \mathbf{H}-\mathbf{N M R}\left(\mathbf{C D C l}_{3}\right.$, $500 \mathrm{MHz}): \delta$ 7.93-7.91 (m, 2H), 7.56-7.53 (m, 1H), 7.47-7.43 (m, 2H), 7.28-7.25 (m, 2H), 7.21-7.18 (m, 3H), $3.15(\mathrm{~d}, J=13.7 \mathrm{~Hz}, 1 \mathrm{H}), 2.67(\mathrm{~d}, J=13.7 \mathrm{~Hz}, 1 \mathrm{H})$ ppm. ${ }^{13} \mathbf{C}-\mathbf{N M R}\left(\mathbf{C D C l}_{3}\right.$, $125 \mathrm{MHz}$ ): $\delta$ 203.9, 166.6, 140.0, 136.5, 133.0, 129.1 (2C), 128.7 (2C), 128.4 (2C), 128.3 (2C), 126.2, 39.2, $29.7 \mathrm{ppm}$.<smiles>Cc1ccccc1C(=O)C(Cc1ccccc1)C(=O)O</smiles>

1f-d $\mathbf{d}_{2} 37 \%$<smiles>[2H]C(Cc1ccccc1)(C(=O)O)C(=O)c1ccccc1C</smiles>

1f- $d_{3} 63 \%$

Chemical Formula: $\mathrm{C}_{17} \mathrm{H}_{16} \mathrm{D}_{2} \mathrm{O}$ Chemical Formula: $\mathrm{C}_{17} \mathrm{H}_{15} \mathrm{D}_{3} \mathrm{O}$

\section{- 2-(methyl- $\left.d_{2}\right)$-3-phenyl-1-(o-tolyl)propan-1-one-2 (1f- $\left.d_{2}+1 \mathrm{f}-d_{3}\right)$}

In a $15 \mathrm{~mL}$ flame-dried Schlenk tube equipped with a stirring bar, the desired ketone $(0.5$ mmol, 1 equiv), benzyl alcohol $\left(0.265 \mathrm{~mL}, 5\right.$ equiv), $\mathrm{KOH}(2.8 \mathrm{mg}, 10 \mathrm{~mol} \%)$, methanol- $d_{4}$ (25 equiv, $0.5 \mathrm{~mL}$ ), iron complex Fe2 (4.56 mg, $2 \mathrm{~mol} \%$ ) and $\mathrm{K}_{3} \mathrm{PO}_{4}(212 \mathrm{mg}, 2$ equiv) were

\footnotetext{
${ }^{8}$ Sklyaruk, J.; Borghs, J-C.; El-Sepelgy, O.; Rueping, M. Angew. Chem. 2019, 131, 785 - 789,5.

${ }^{9}$ Lator, A.; Gaillard, S.; Poater, A.; Renaud, J-L. Chem. Eur. J, 2018, 24, 5770 - 5774.
} 
poured in under an argon atmosphere. The mixture was rapidly stirred at room temperature for $2 \mathrm{~min}$ and then placed into a pre-heated oil bath at $90^{\circ} \mathrm{C}$ and stirred over 24 hours. The mixture was cooled-down to room temperature, filtrated over silica with diethyl ether and concentrated under reduced pressure. The conversion was determined by ${ }^{1} \mathrm{H}-\mathrm{NMR}$ spectroscopy, and then, the residue was purified by flash chromatography on silica gel using pentane-ethyl acetate (95:5) as eluent to afford the desired product as a colorless oil $(98 \mathrm{mg}$, $81 \%)$. 1f- $\boldsymbol{d}_{2}:{ }^{1} \mathrm{H}-\mathrm{NMR}\left(\mathbf{C D C l}_{3}, \mathbf{5 0 0} \mathbf{~ M H z}\right): \delta 7.38(\operatorname{app~dd}, J=6.3 ; 1.0 \mathrm{~Hz}, 1 \mathrm{H}), 7.31$ (td, $\mathrm{J}=$ 6.3; $1.0 \mathrm{~Hz}, 1 \mathrm{H}), 7.27-7.24(\mathrm{~m}, 3 \mathrm{H}), 7.22-7.16(\mathrm{~m}, 5 \mathrm{H}), 3.52($ app q, $J=5.9 \mathrm{~Hz}, 1 \mathrm{H}), 3.13$ (app dd, $J=11.4 ; 6.5 \mathrm{~Hz}, 1 \mathrm{H}$ ), 2.61 (app dd, $J=11.4 ; 6.2 \mathrm{~Hz}, 1 \mathrm{H}), 2.39$ (s, 3H), 1.11 (app d, $J=5.9 \mathrm{~Hz}, 1 \mathrm{H})$ ppm. ${ }^{13} \mathrm{C}-\mathrm{NMR}\left(\mathrm{CDCl}_{3}, 125 \mathrm{MHz}\right): \delta 208.2(\mathrm{~d}, J=2.5 \mathrm{~Hz}), 140.0$ (d, $J=1.0$ $\mathrm{Hz}), 138.6(\mathrm{~d}, J=3.3 \mathrm{~Hz}), 137.8(\mathrm{~d}, J=2.6 \mathrm{~Hz}), 131.7(\mathrm{~d}, J=1.0 \mathrm{~Hz}), 130.8(\mathrm{~d}, J=2.0 \mathrm{~Hz})$, 129.1 (d, $J=1.0 \mathrm{~Hz}, 2 \mathrm{C}), 128.4(\mathrm{~d}, J=6.2 \mathrm{~Hz}, 2 \mathrm{C}), 127.6$ (d, $J=2.3 \mathrm{~Hz}), 126.2,125.6,46.2$, 39.1 (d, $J=7.8 \mathrm{~Hz}$ ), 20.8 (d, $J=1.1 \mathrm{~Hz}$ ), 16.7-16.0 (m) ppm. IR (neat): 3062, 3026, 2927, 2224, 1681, 1601, 1571, 1495, 1454, 1253, 1078, 1030, 936, 730, 699, 456. HRMS [M+H] : calculated $\mathrm{C}_{17} \mathrm{H}_{17} \mathrm{D}_{2} \mathrm{O}$ 241.1561; found: 241.1543. 1f- $\mathrm{d}_{3}:{ }^{1} \mathrm{H}-\mathrm{NMR}\left(\mathrm{CDCl}_{3}, \mathbf{5 0 0} \mathbf{~ M H z}\right): \delta 7.38$ (app dd, $J=6.3 ; 1.0 \mathrm{~Hz}, 1 \mathrm{H}), 7.31(\mathrm{td}, J=6.3 ; 1.0 \mathrm{~Hz}, 1 \mathrm{H}), 7.27-7.24(\mathrm{~m}, 3 \mathrm{H}), 7.22-7.16(\mathrm{~m}$, $5 \mathrm{H}$ ), 3.13 (app dd, $J=11.4 ; 6.5 \mathrm{~Hz}, 1 \mathrm{H}$ ), 2.61 (app dd, $J=11.4 ; 6.2 \mathrm{~Hz}, 1 \mathrm{H}), 2.39(\mathrm{~s}, 3 \mathrm{H})$, 1.11 (app d, $J=5.9 \mathrm{~Hz}, 1 \mathrm{H}$ ) ppm. ${ }^{13} \mathrm{C}$-NMR $\left(\mathrm{CDCl}_{3}, 125 \mathrm{MHz}\right): \delta 208.2(\mathrm{~d}, J=2.5 \mathrm{~Hz}$ ), 140.0 $(\mathrm{d}, J=1.0 \mathrm{~Hz}), 138.6(\mathrm{~d}, J=3.3 \mathrm{~Hz}), 137.8(\mathrm{~d}, J=2.6 \mathrm{~Hz}), 131.7(\mathrm{~d}, J=1.0 \mathrm{~Hz}), 130.8(\mathrm{~d}, J$ $=2.0 \mathrm{~Hz}$ ), $129.1(\mathrm{~d}, J=1.0 \mathrm{~Hz}, 2 \mathrm{C}), 128.4(\mathrm{~d}, J=6.2 \mathrm{~Hz}, 2 \mathrm{C}), 127.6(\mathrm{~d}, J=2.3 \mathrm{~Hz}), 126.2$, 125.6, $45.6(\mathrm{t}, J=16.4 \mathrm{~Hz}), 39.0(\mathrm{~d}, J=7.8 \mathrm{~Hz}), 20.8(\mathrm{~d}, J=1.1 \mathrm{~Hz}), 16.7-16.0(\mathrm{~m}) \mathrm{ppm}$. HRMS [M+H] $]^{+}$: calculated $\mathrm{C}_{17} \mathrm{H}_{16} \mathrm{D}_{3} \mathrm{O} 242.1624$; found: 242.1618 . 
Part 5: NMR Spectra

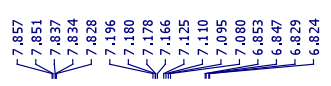

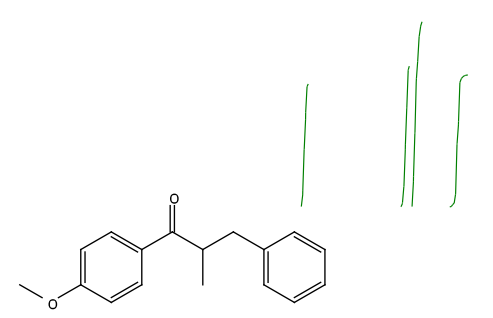

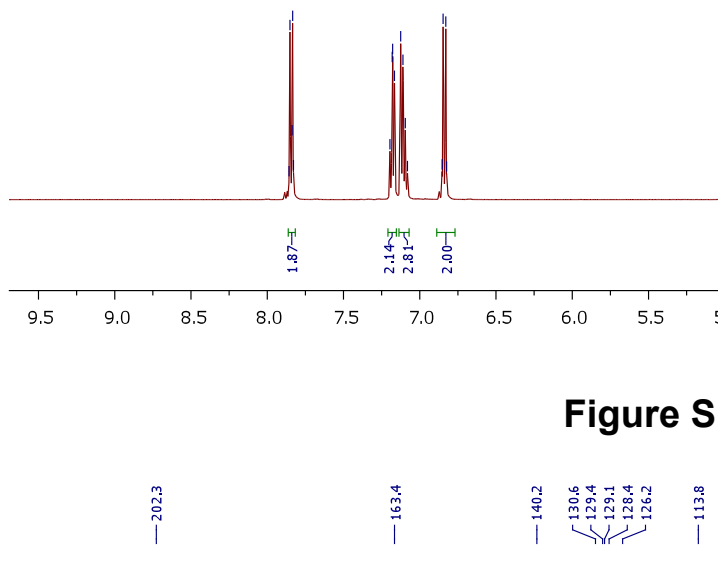
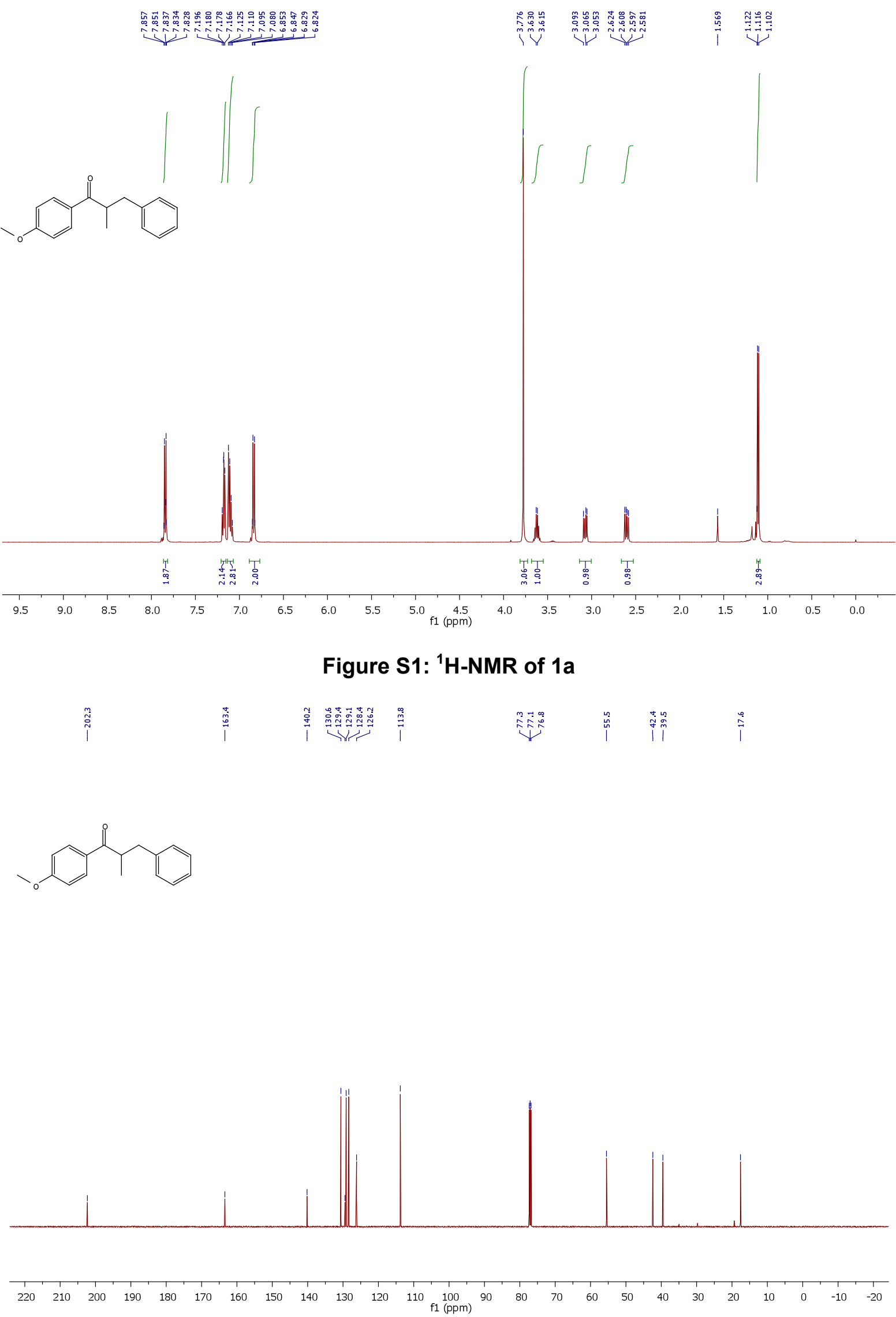

Figure S2: ${ }^{13} \mathrm{C}-\mathrm{NMR}$ of $1 \mathrm{a}$

S-23 

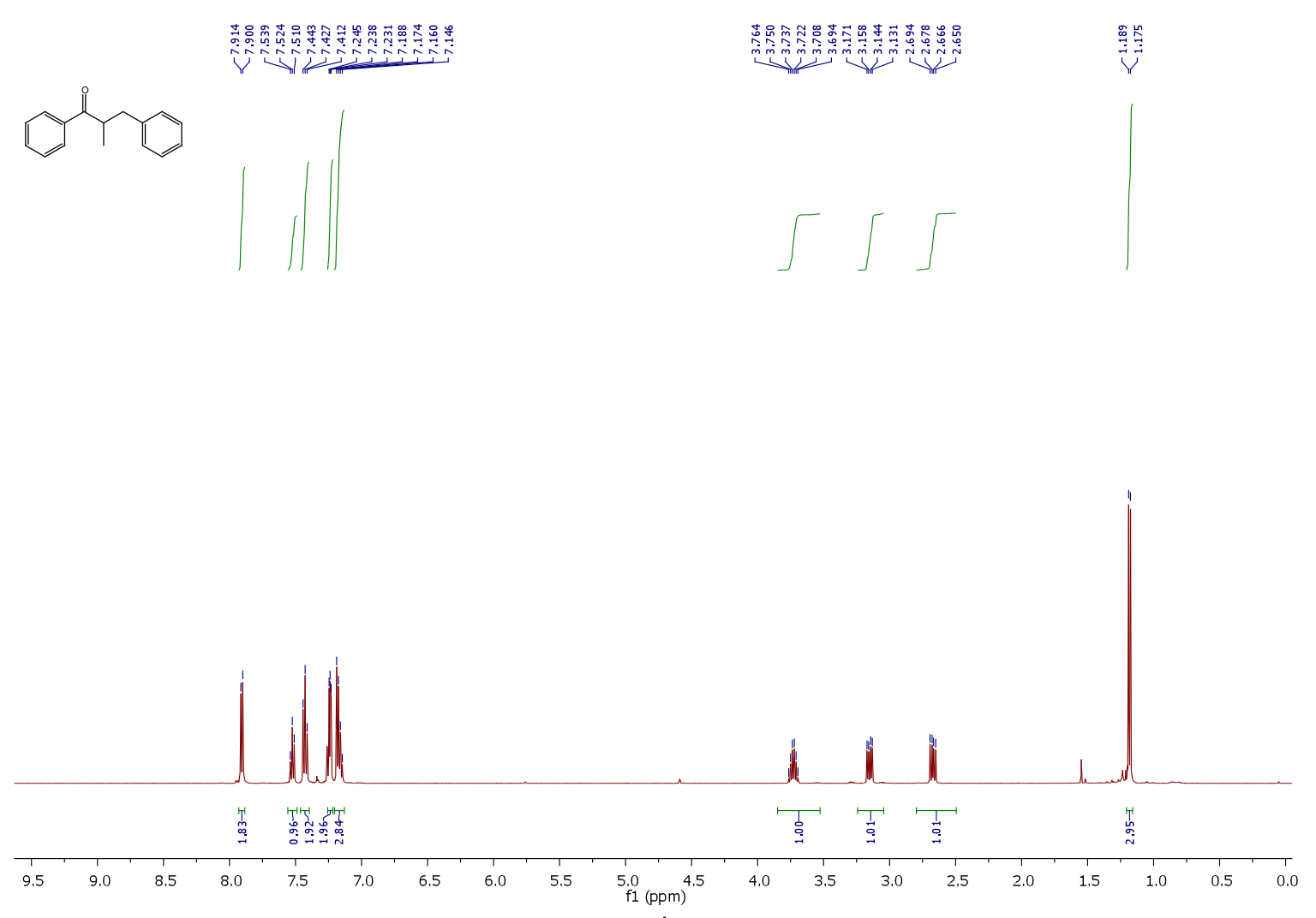

Figure S3: ${ }^{1} \mathrm{H}-\mathrm{NMR}$ of $1 \mathrm{~b}$

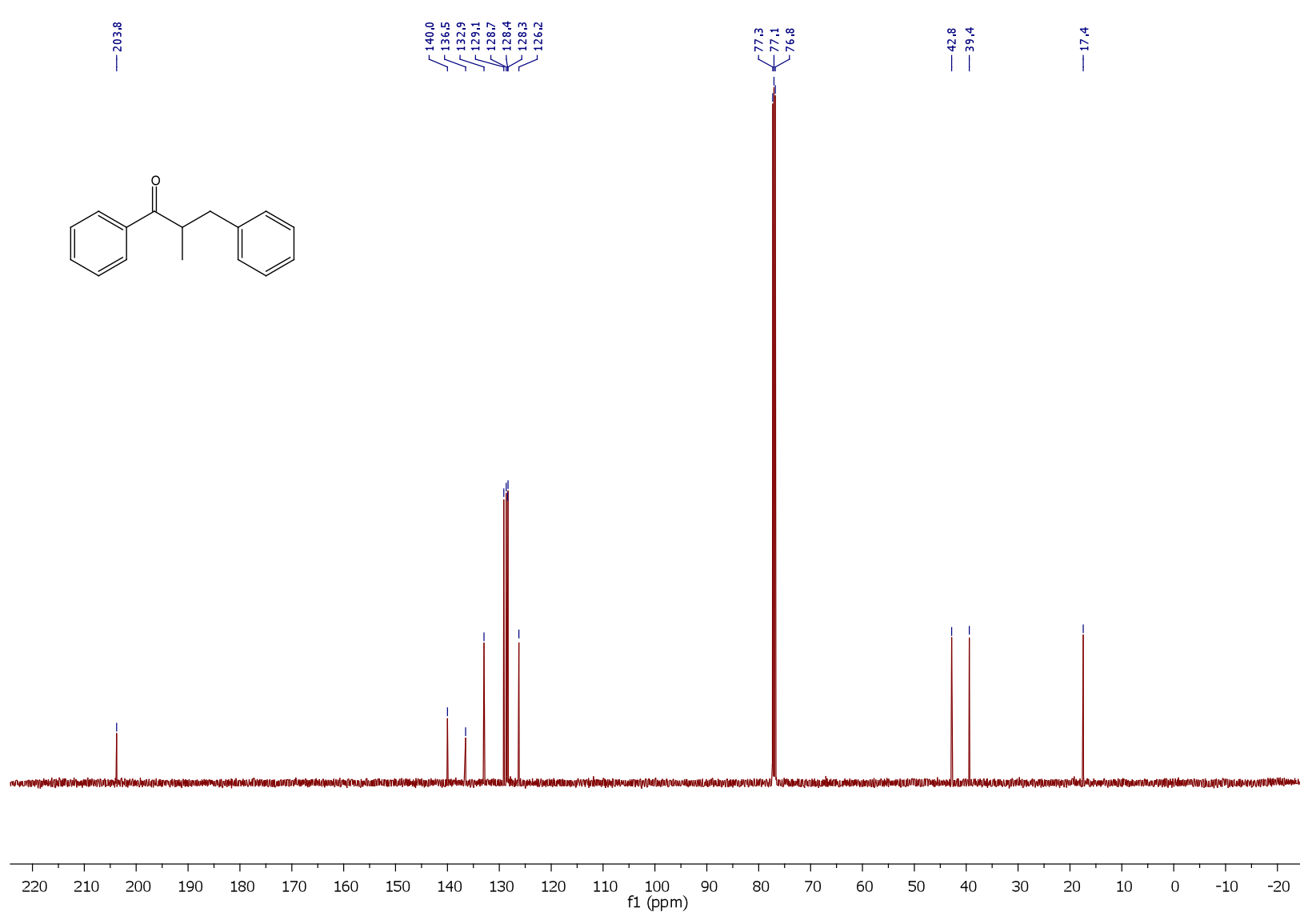

Figure S4: ${ }^{13} \mathrm{C}-\mathrm{NMR}$ of $1 \mathrm{~b}$ 


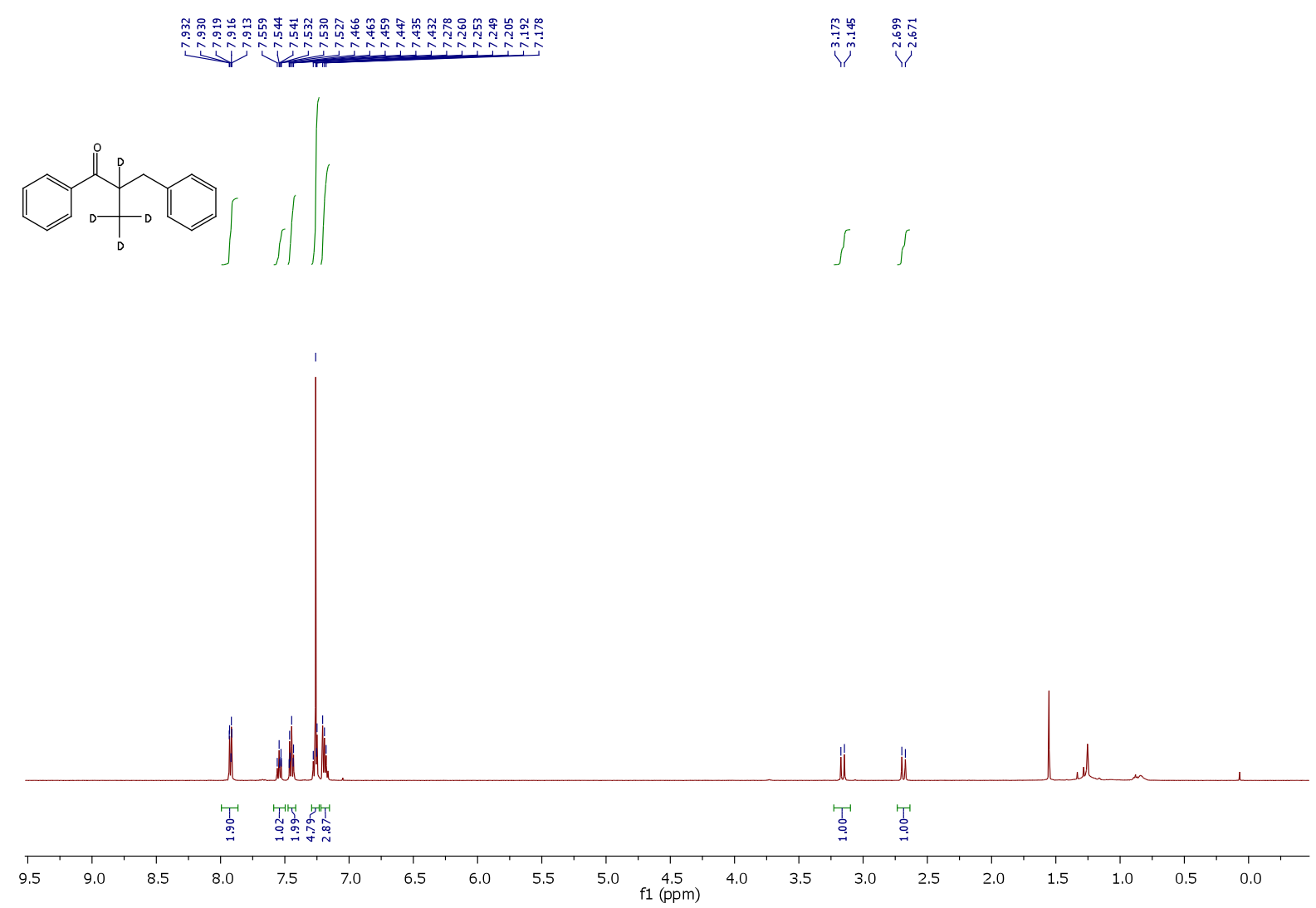

Figure S5: ${ }^{1} \mathrm{H}-\mathrm{NMR}$ of $1 \mathrm{~b}-\mathrm{d}_{4}$
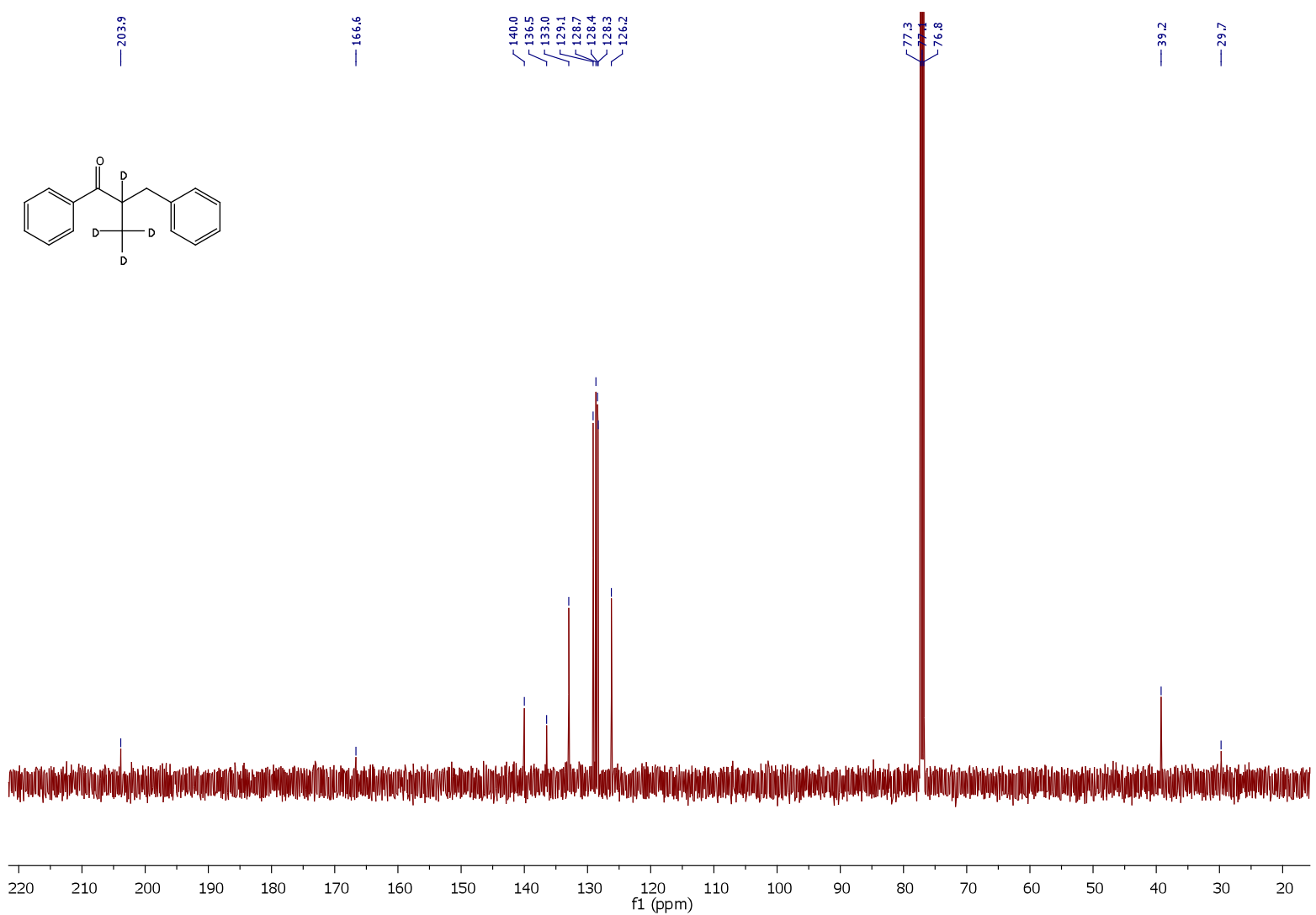

Figure S6: ${ }^{13} \mathrm{C}-\mathrm{NMR}$ of $1 \mathrm{~b}-\mathrm{d}_{4}$ 

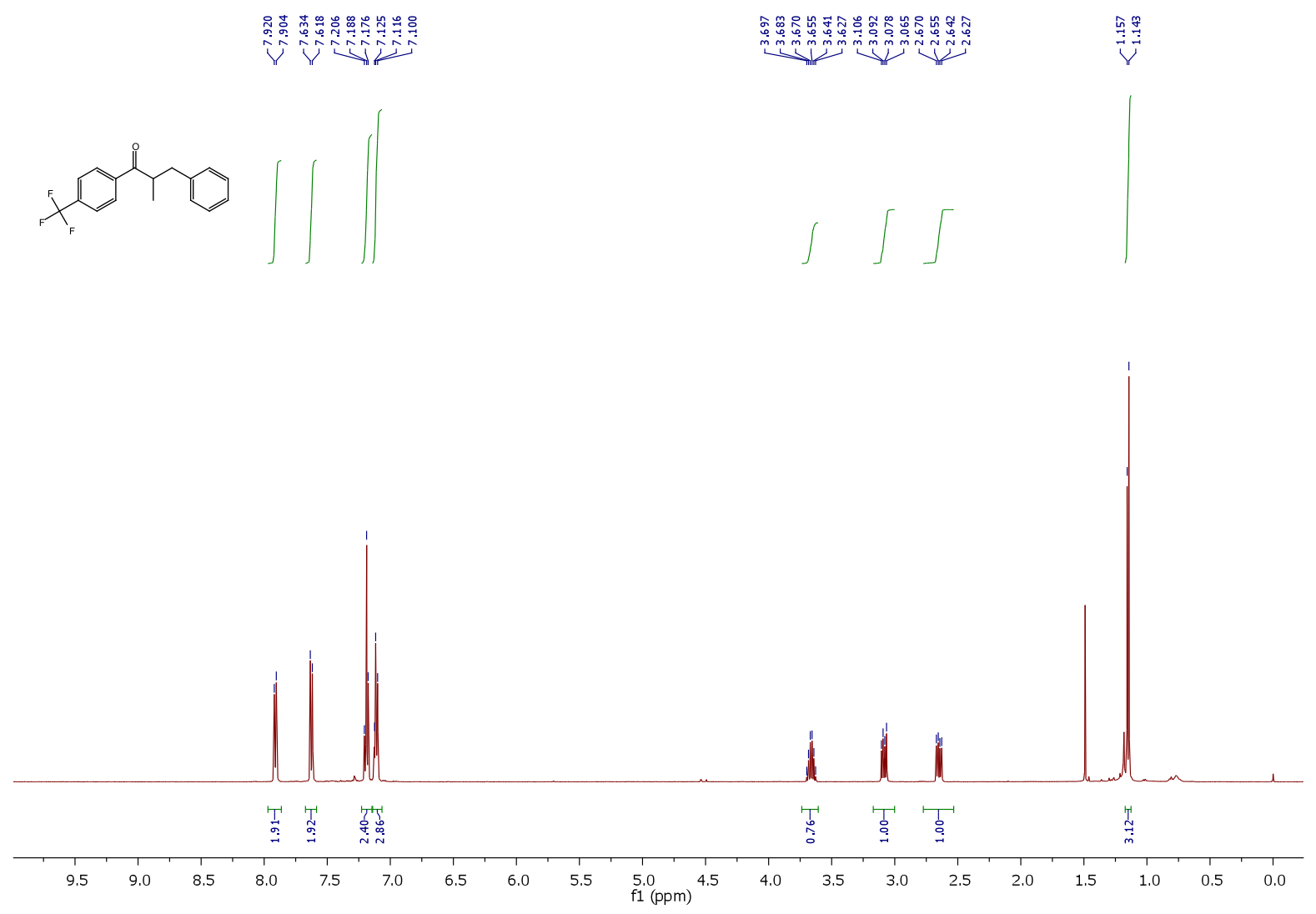

Figure S7: ${ }^{1} \mathrm{H}-\mathrm{NMR}$ of $1 \mathrm{C}$

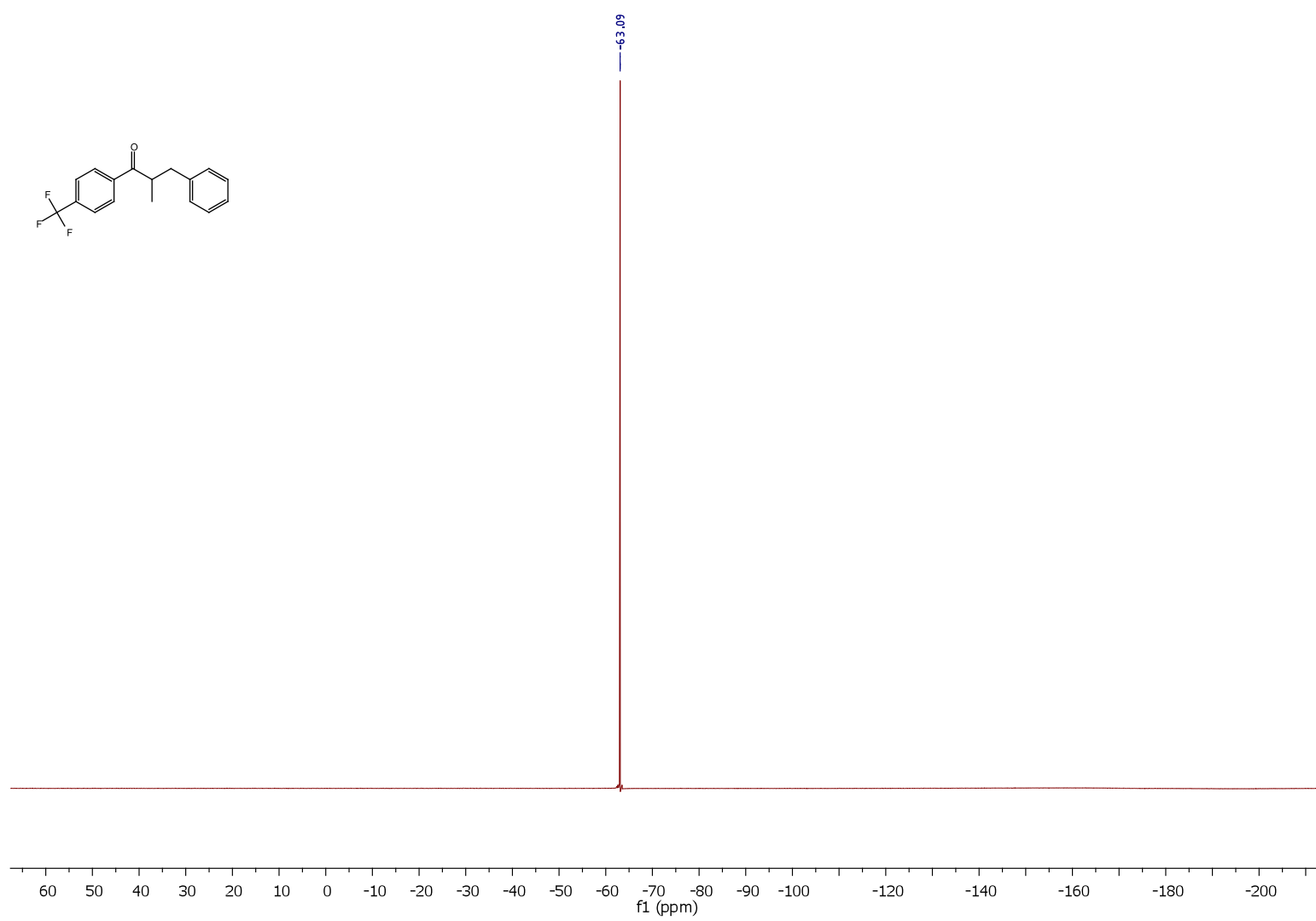


Figure S8: ${ }^{19} \mathrm{~F}-\mathrm{NMR}$ of $1 \mathrm{C}$

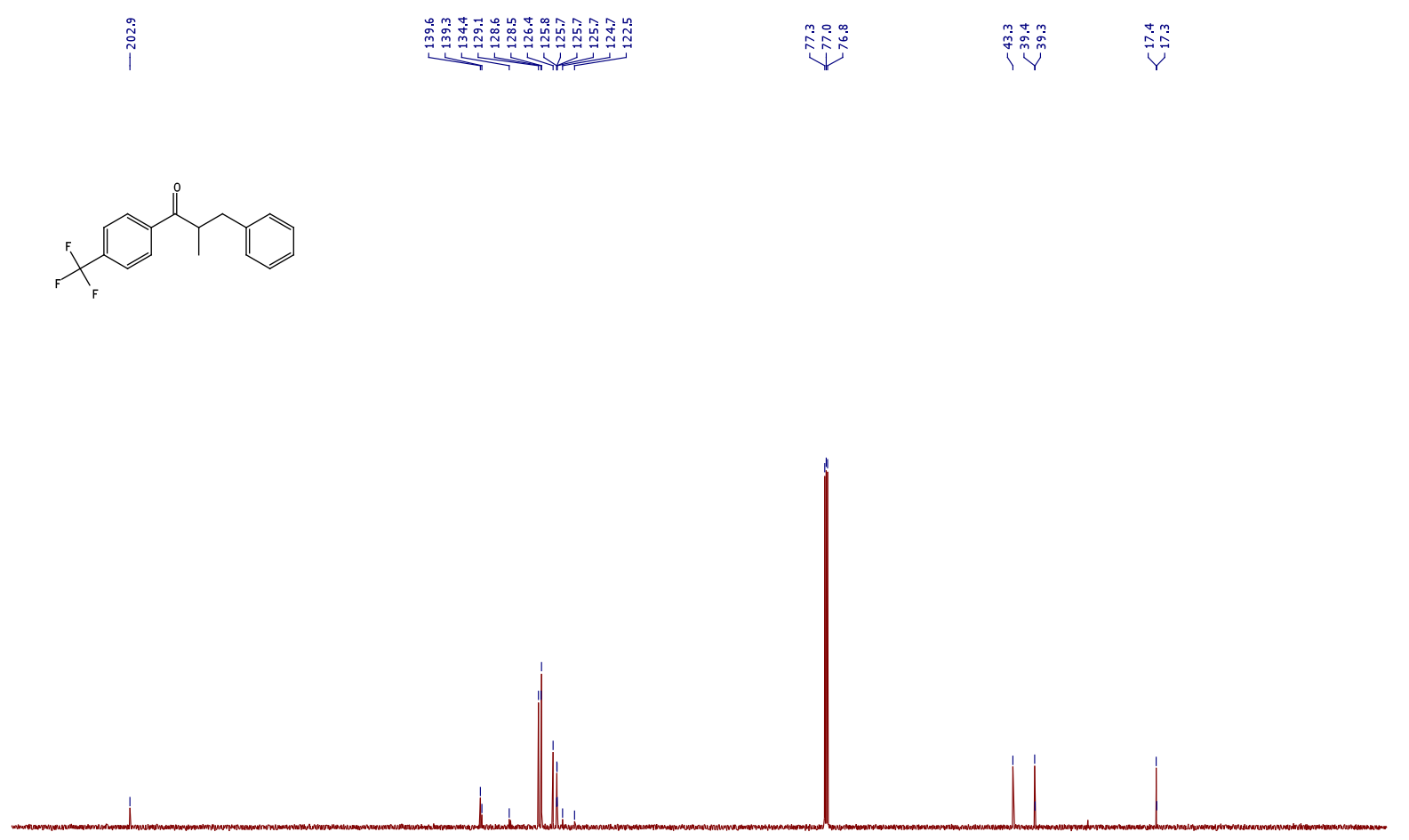

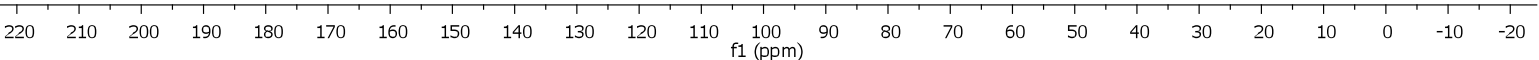

Figure S9: ${ }^{13} \mathrm{C}-\mathrm{NMR}$ of $1 \mathrm{C}$

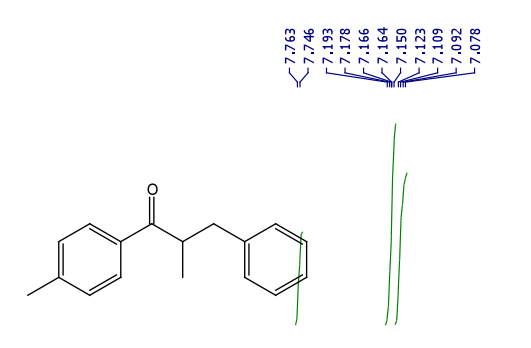

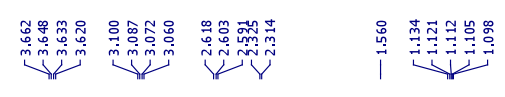

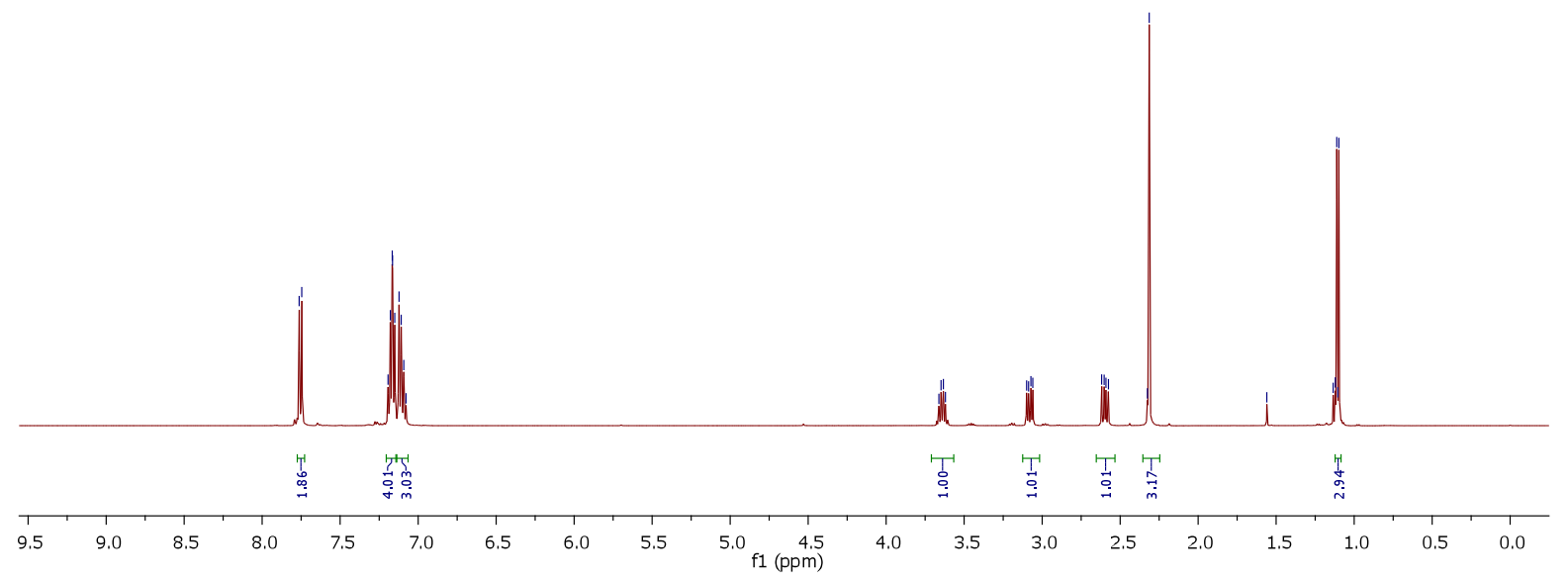


Figure S10: ${ }^{1} \mathrm{H}-\mathrm{NMR}$ of $1 \mathrm{~d}$

i্⿳亠丷厂巾

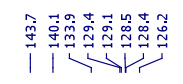

$\sqrt[n]{n=\infty}$

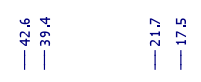
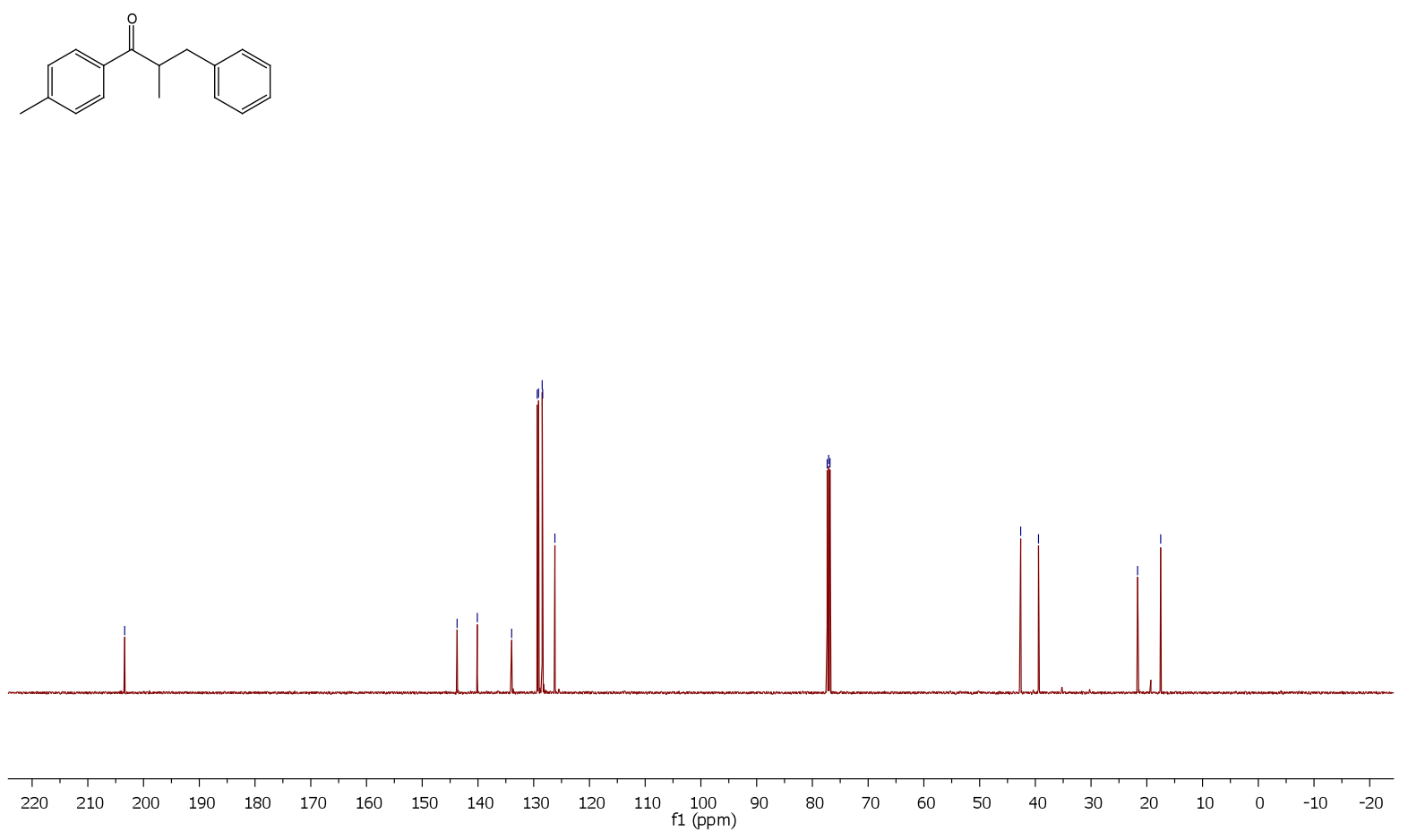

Figure S11: ${ }^{13} \mathrm{C}-\mathrm{NMR}$ of $1 \mathrm{~d}$
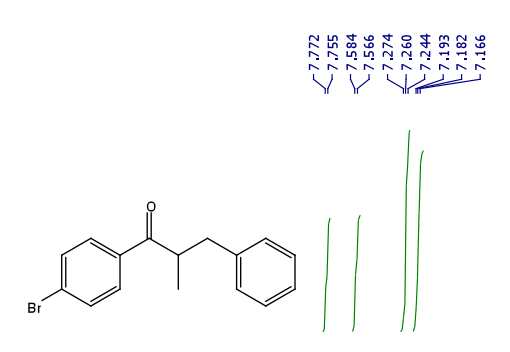
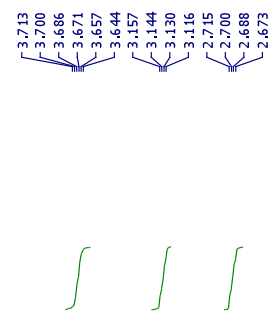

Vับ

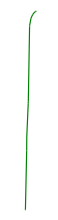

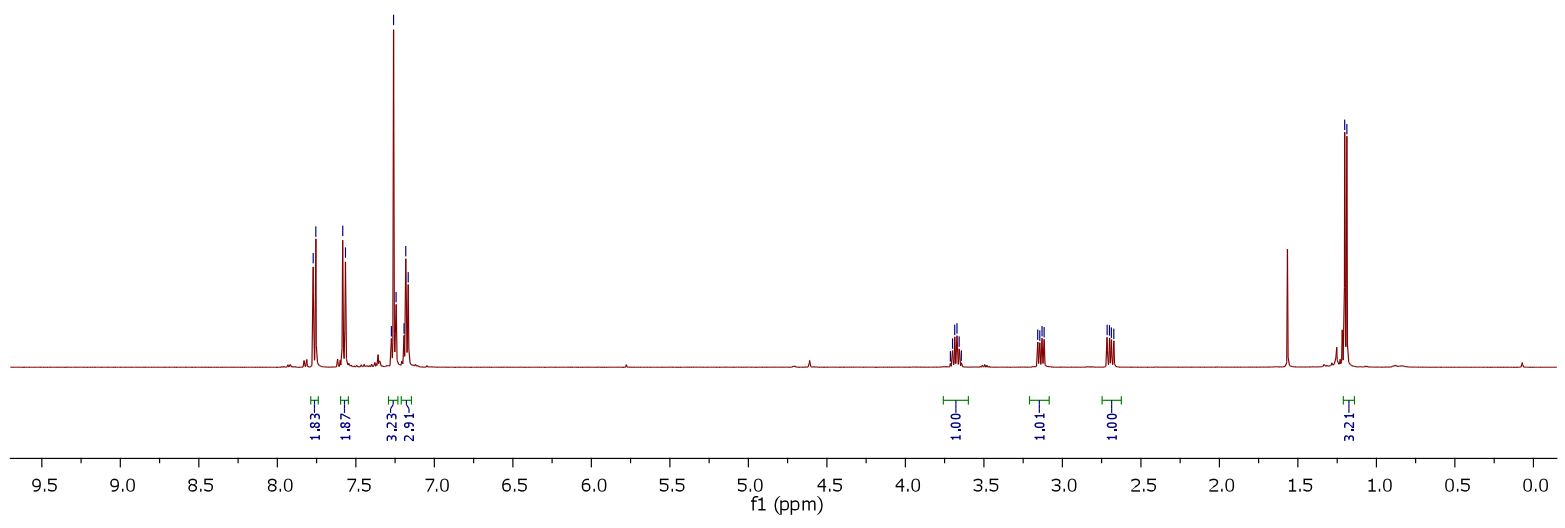

Figure S12: ${ }^{1} \mathrm{H}-\mathrm{NMR}$ of $1 \mathrm{e}$

S-28 
$\stackrel{\infty}{\stackrel{\sim}{i}}$

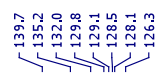

$\sum_{\substack{n+\infty \\ n=0}}^{n-\infty}$

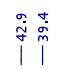

$\stackrel{n}{i}$
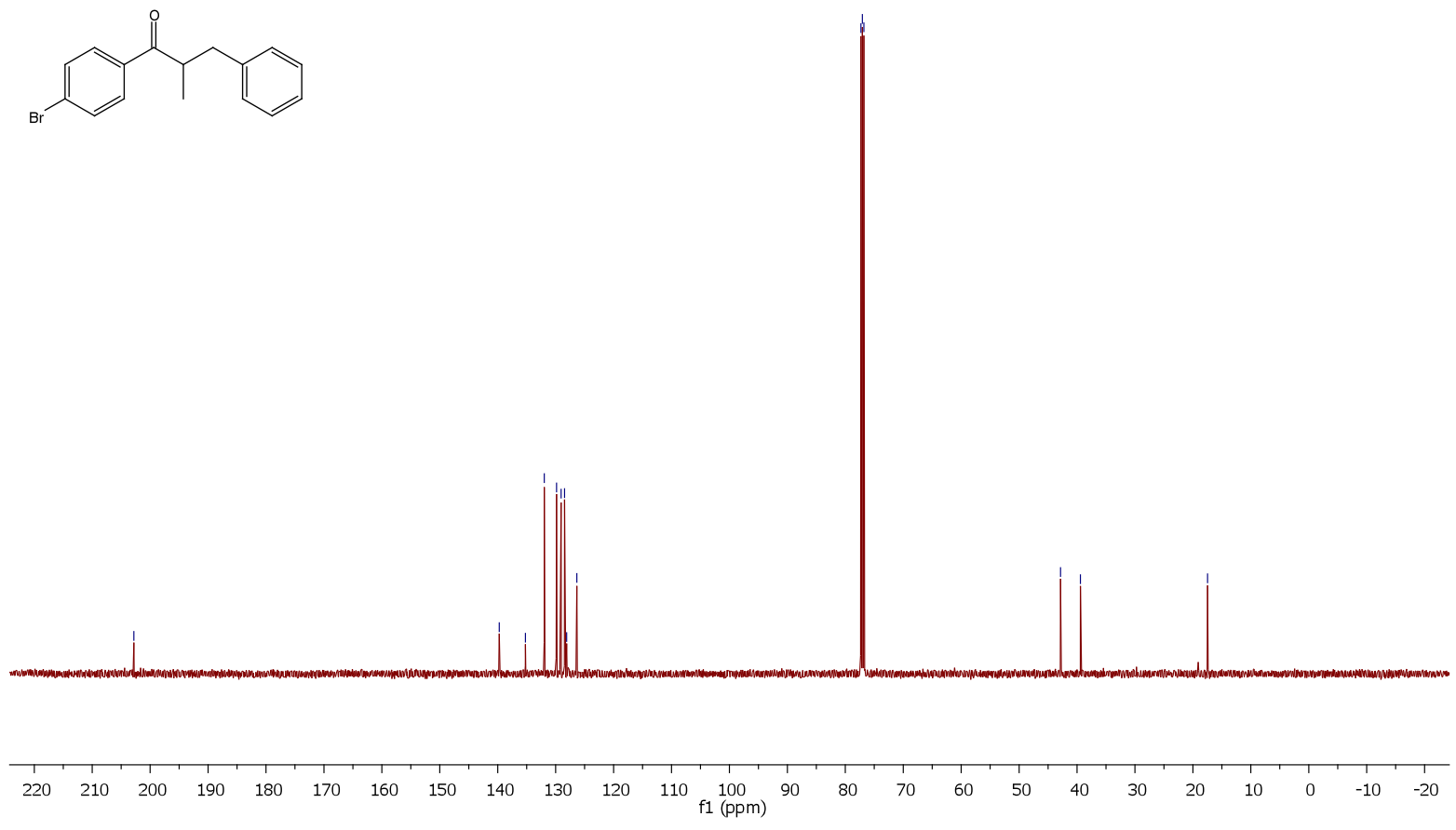

Figure S13: ${ }^{13} \mathrm{C}$-NMR of $1 \mathrm{e}$

S-29 


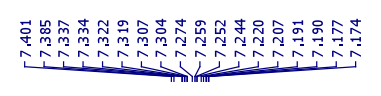
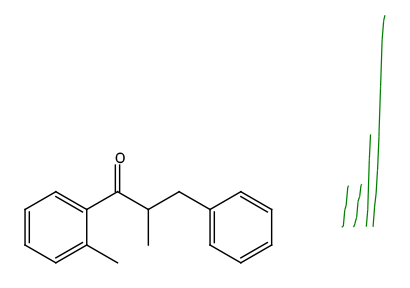

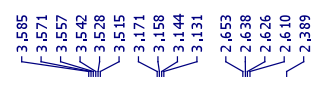

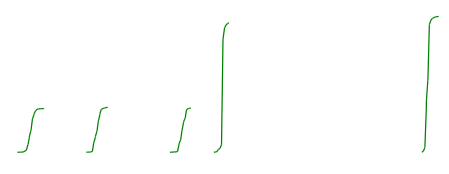

品

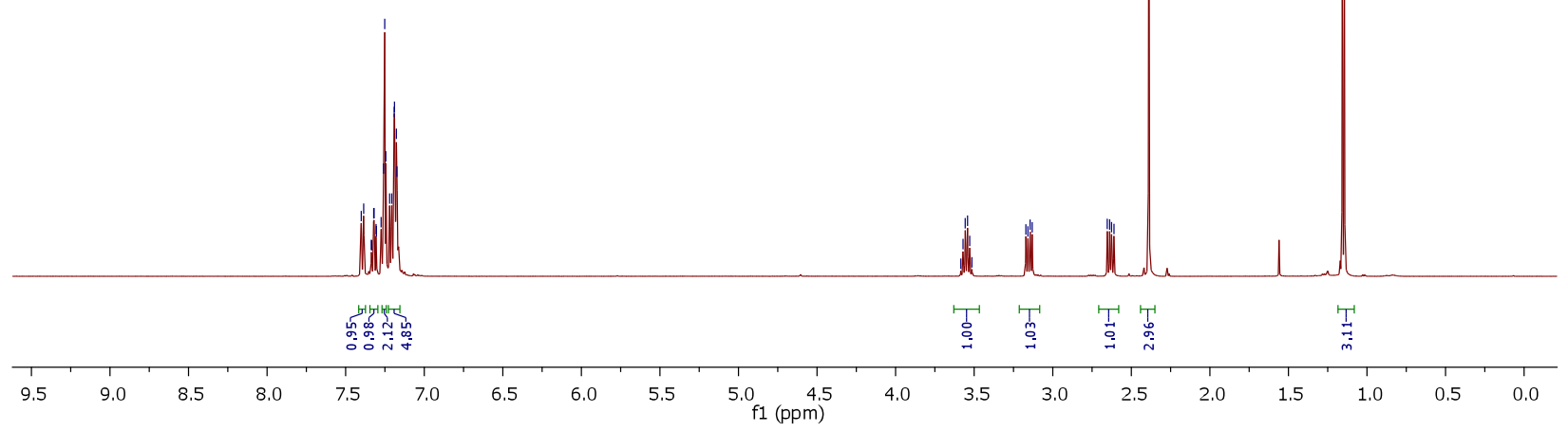

Figure S14: ${ }^{1} \mathrm{H}-\mathrm{NMR}$ of $1 \mathrm{f}$

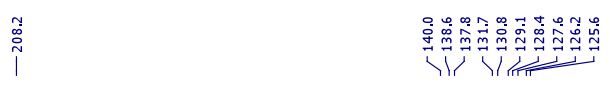

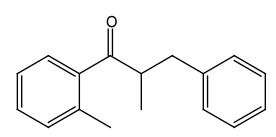

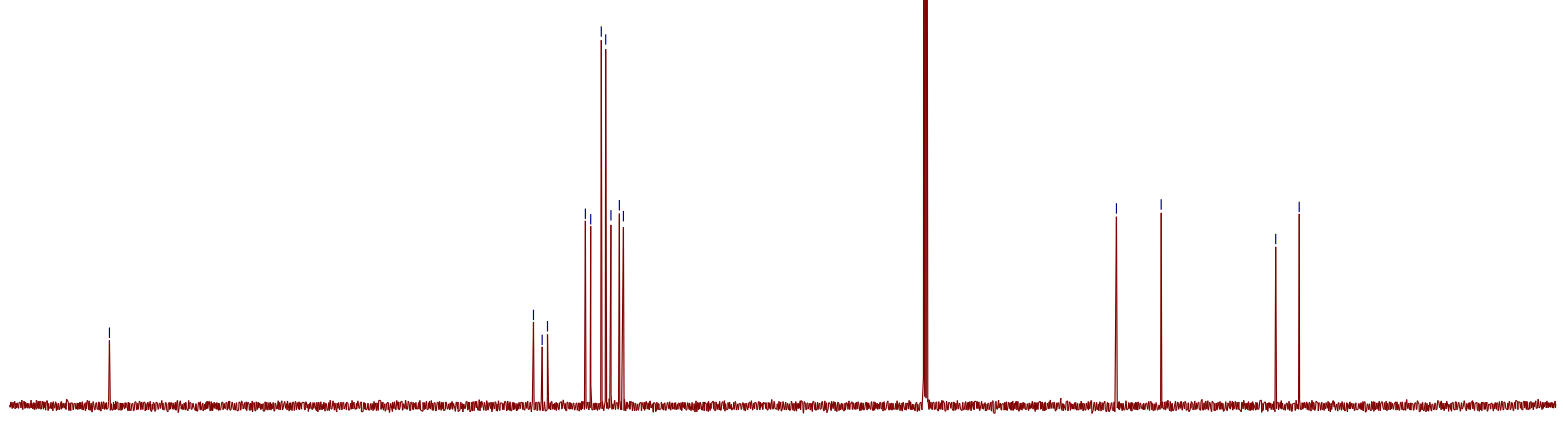

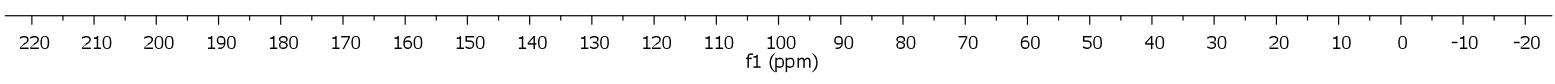

Figure $\mathrm{S} 15:{ }^{13} \mathrm{C}$-NMR of $1 \mathrm{f}$

S-30 


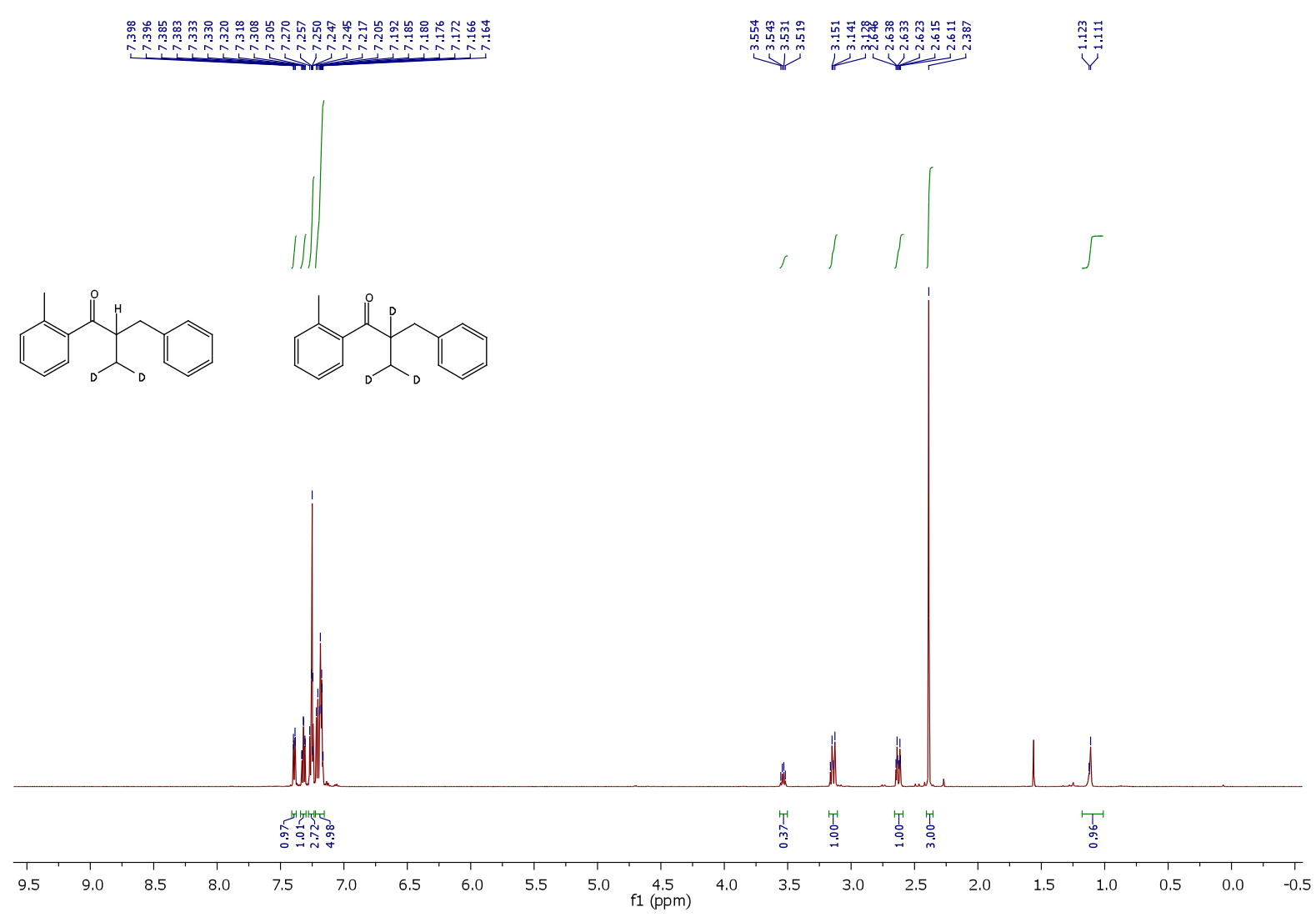

Figure S16: ${ }^{1} \mathrm{H}-\mathrm{NMR}$ of $1 \mathrm{f}-\mathrm{d}_{2}+1 \mathrm{f}-\mathrm{d}_{3}$

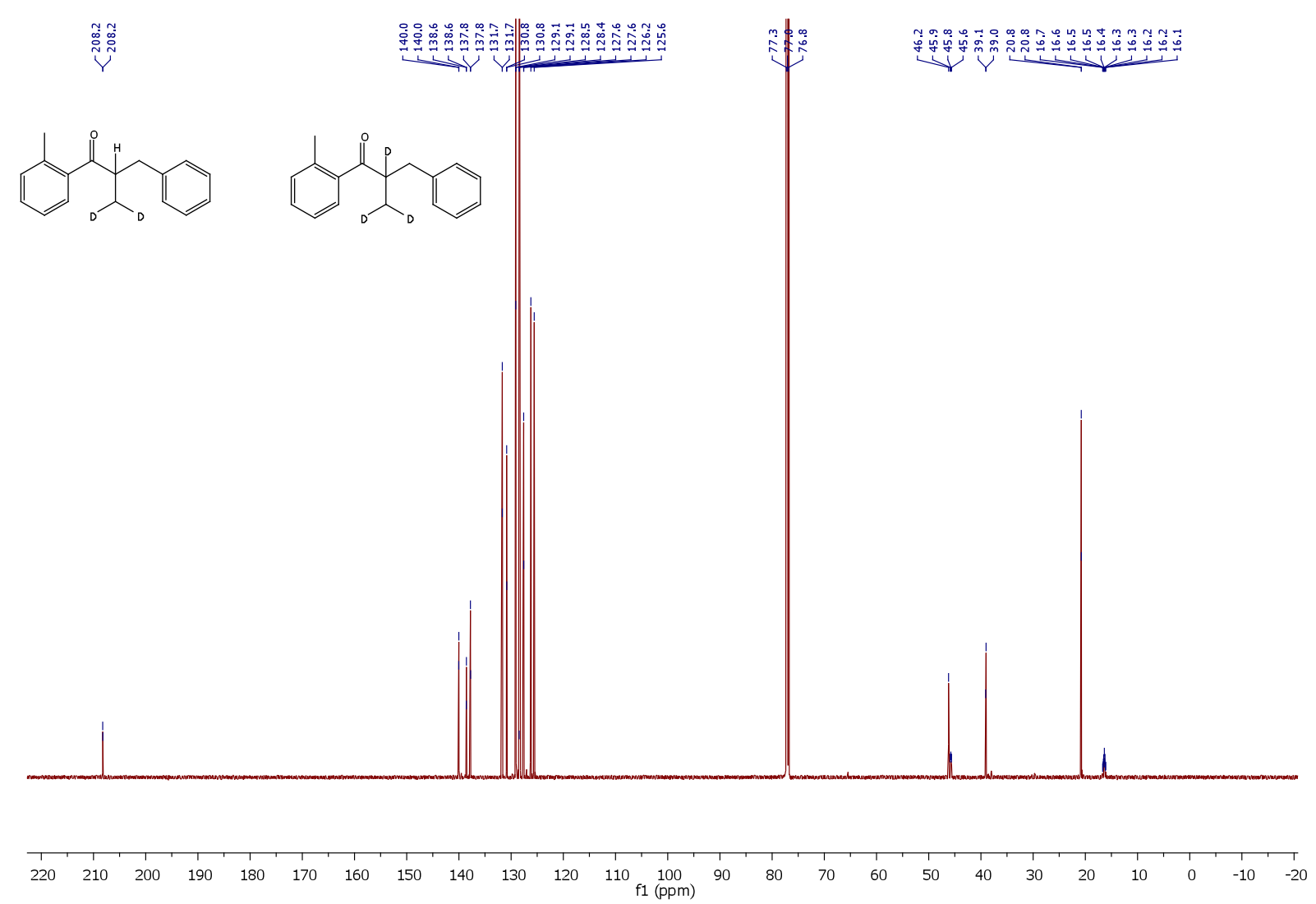

Figure S17: ${ }^{13} \mathrm{C}-\mathrm{NMR}$ of $1 \mathrm{f}-\mathrm{d}_{2}+1 \mathrm{f}-\mathrm{d}_{3}$ 


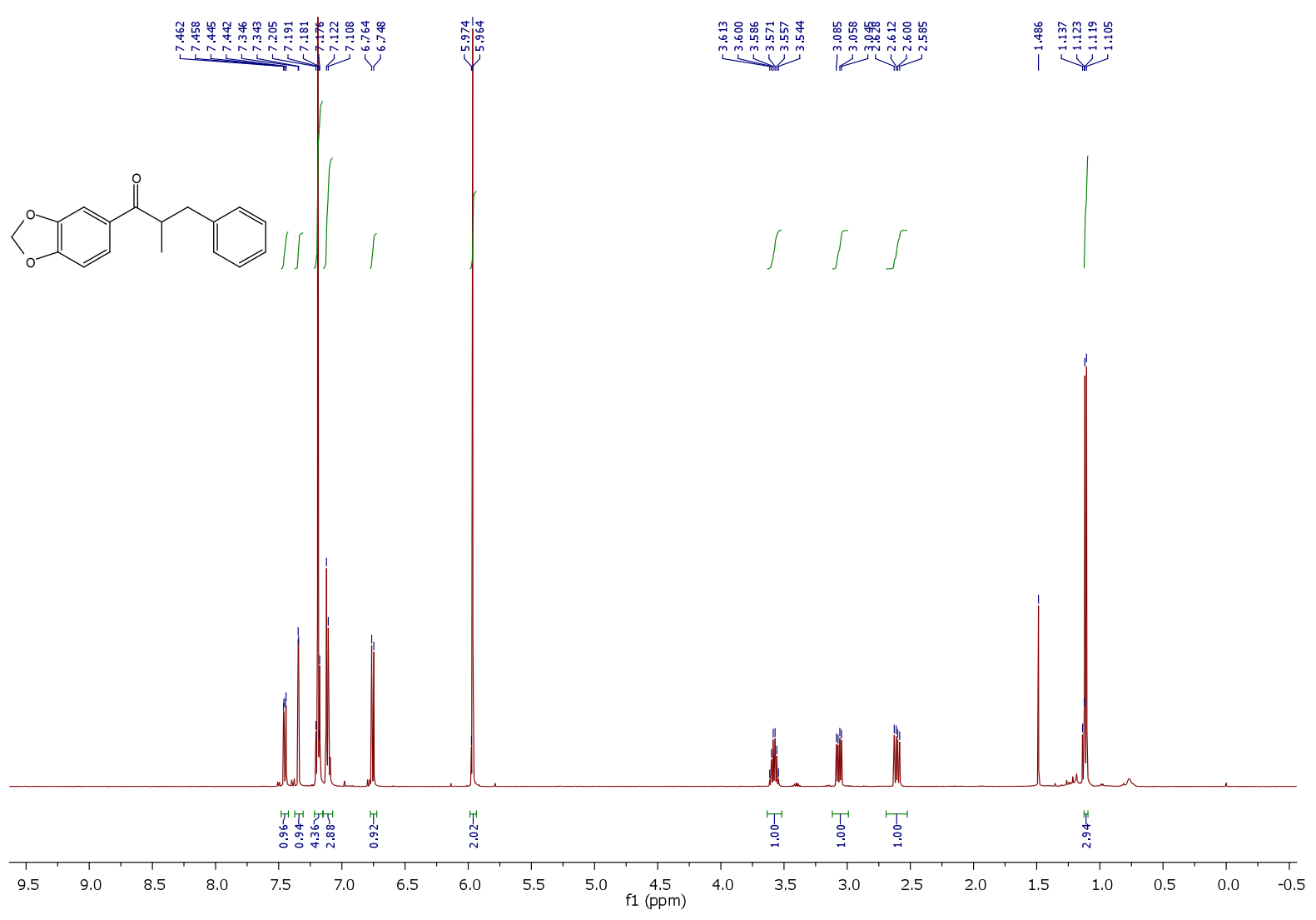

Figure S18: ${ }^{1} \mathrm{H}-\mathrm{NMR}$ of $1 \mathrm{~g}$
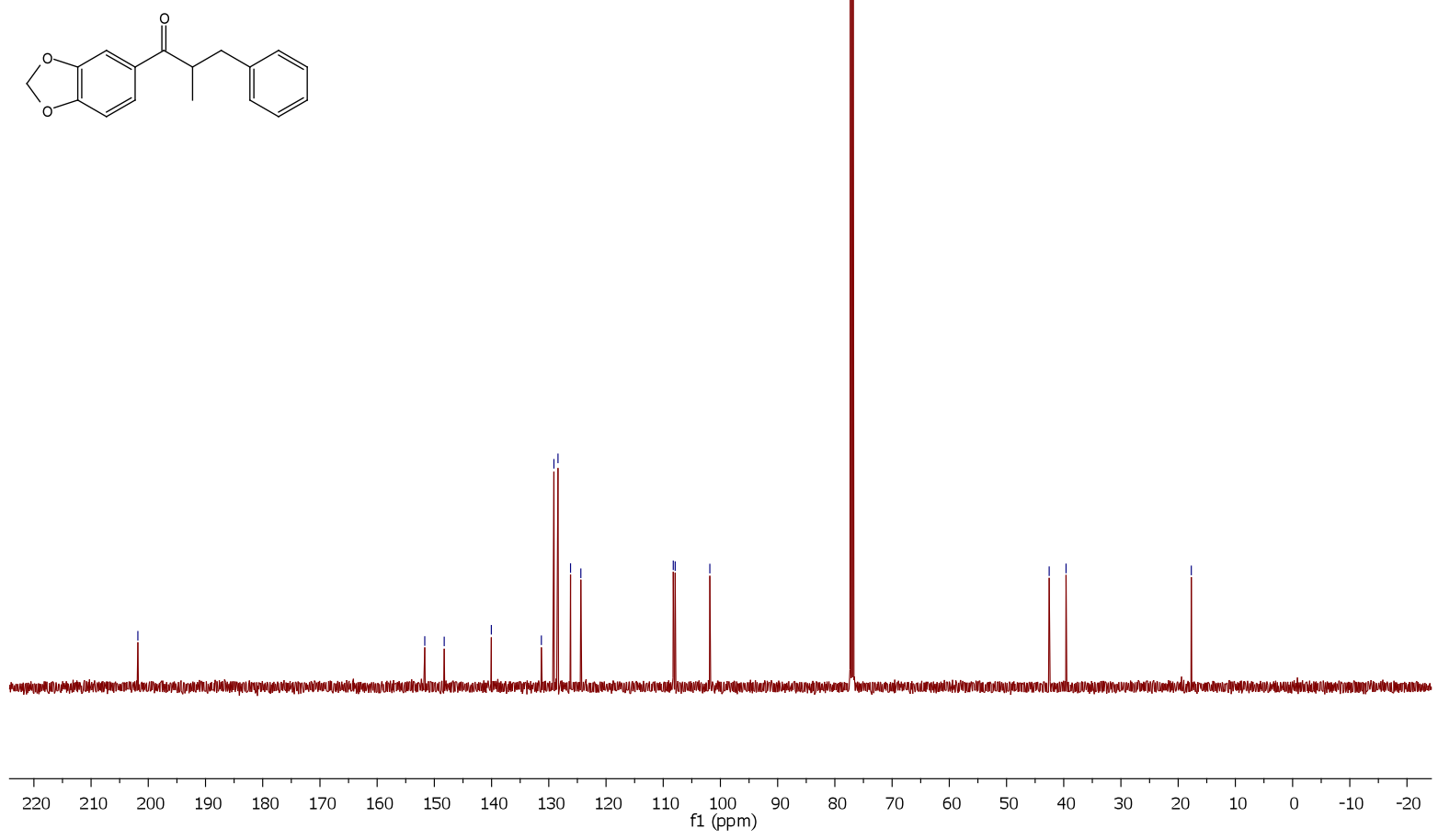

Figure S19: ${ }^{13} \mathrm{C}-\mathrm{NMR}$ of $1 \mathrm{~g}$

S-32 


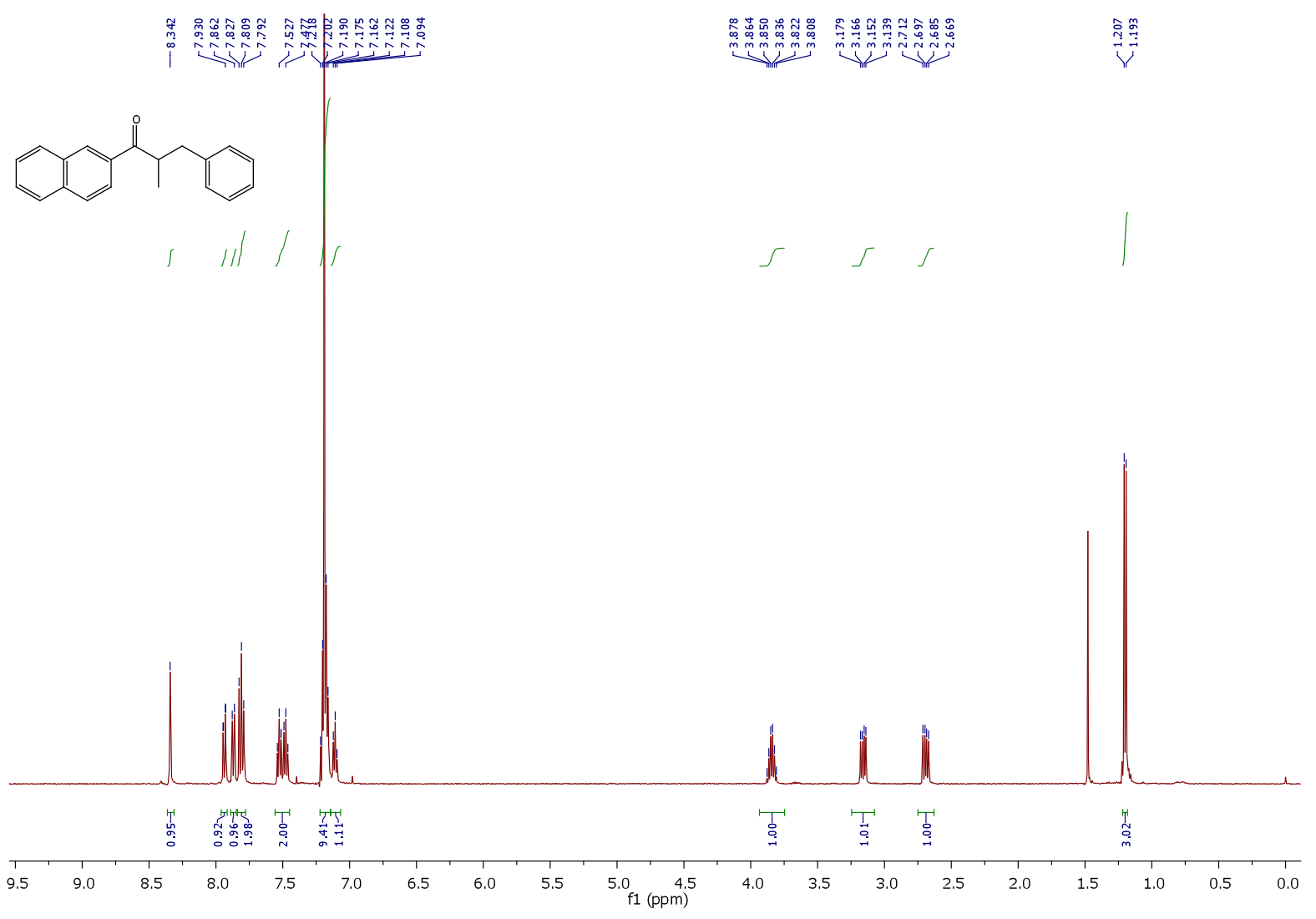

Figure S20: ${ }^{1} \mathrm{H}-\mathrm{NMR}$ of $1 \mathrm{~h}$

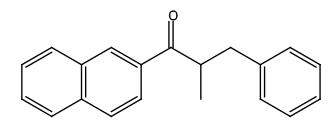

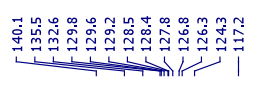

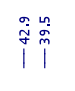
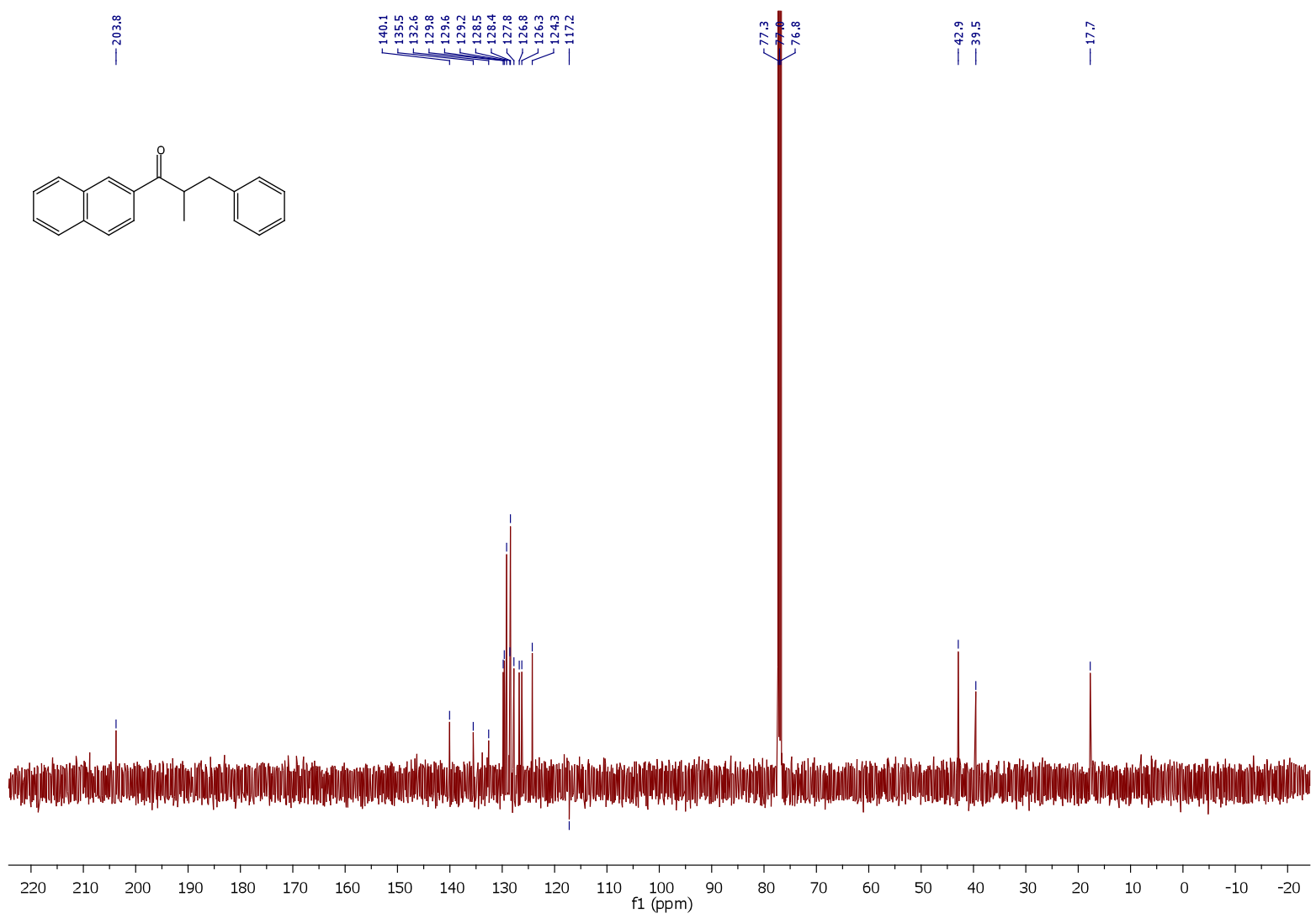

Figure S21: ${ }^{13} \mathrm{C}-\mathrm{NMR}$ of $1 \mathrm{~h}$

S-33 


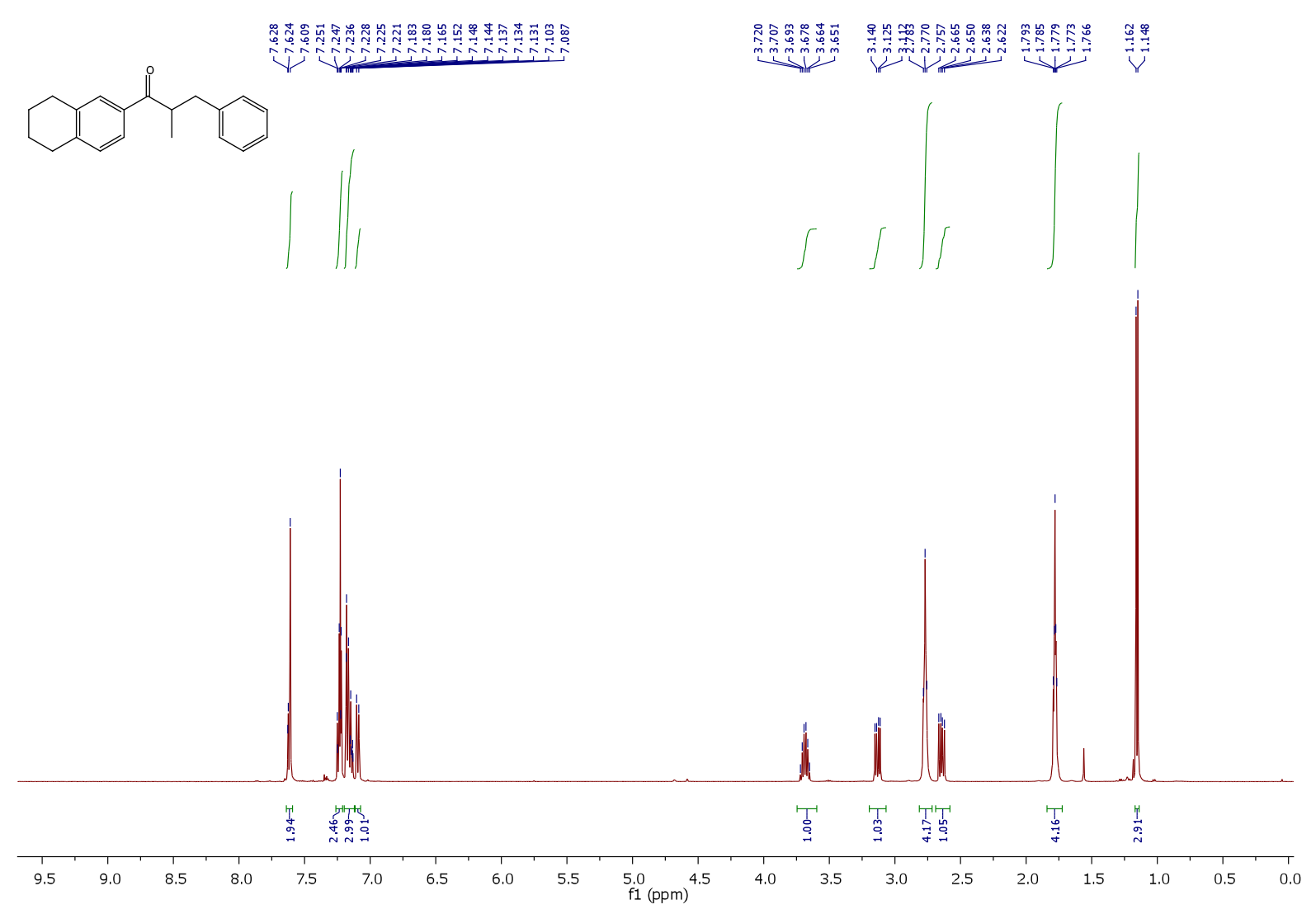

Figure S22: ${ }^{1} \mathrm{H}-\mathrm{NMR}$ of $1 \mathrm{i}$

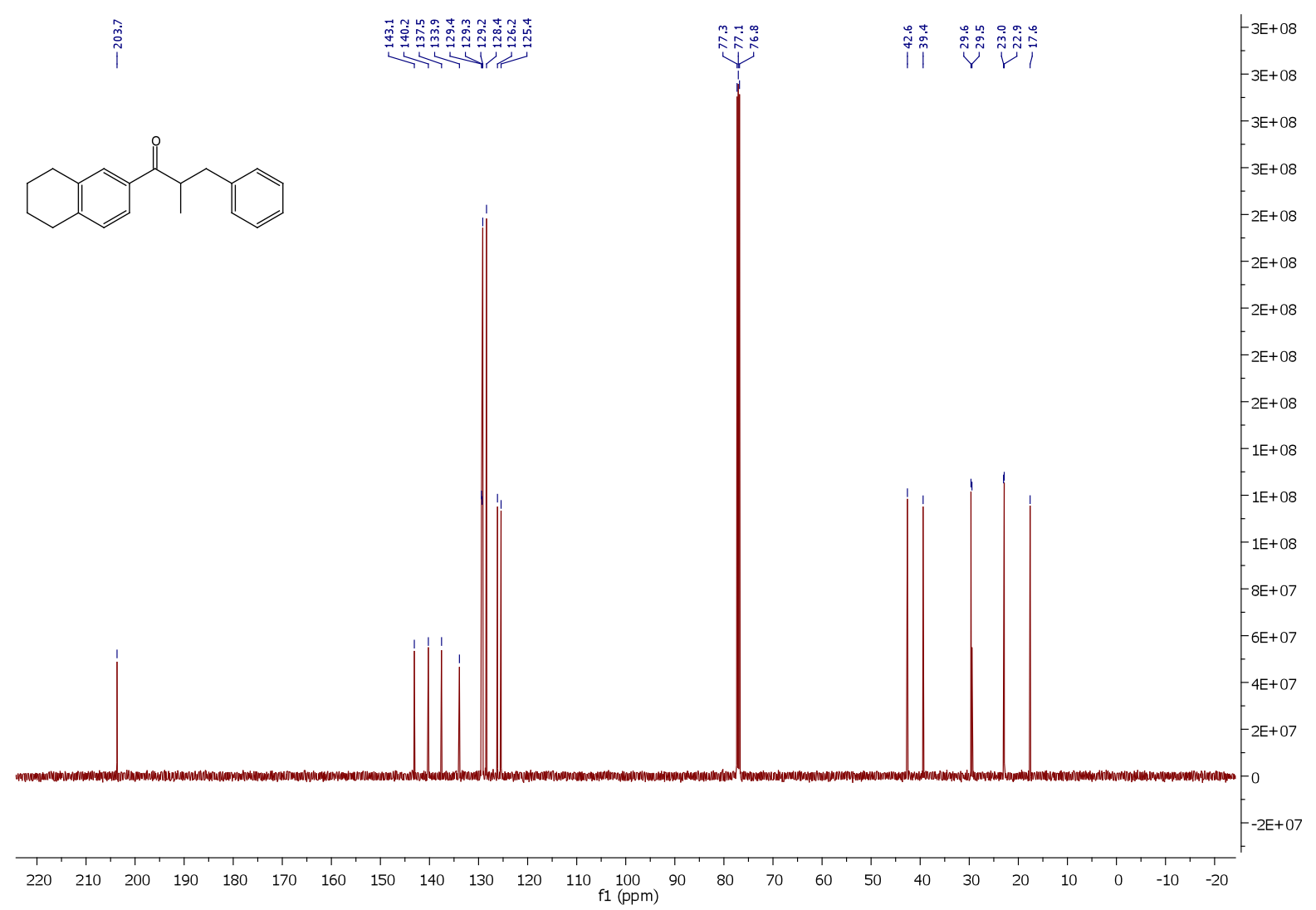

Figure S23: ${ }^{13} \mathrm{C}-\mathrm{NMR}$ of $1 \mathrm{i}$ 


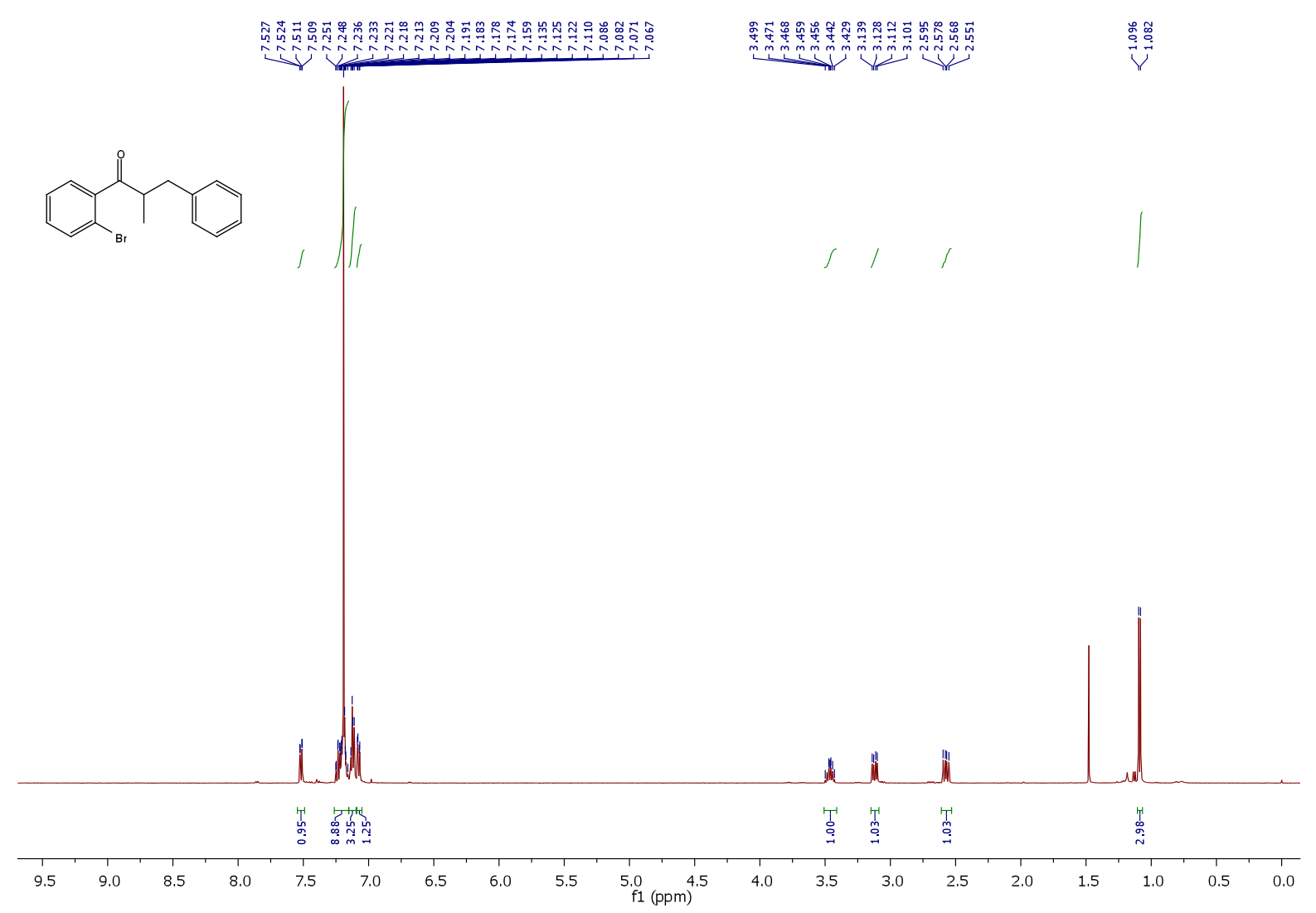

Figure S24: ${ }^{1} \mathrm{H}-\mathrm{NMR}$ of $1 \mathrm{j}$

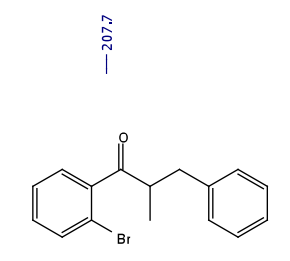

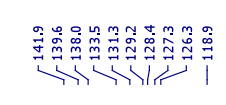

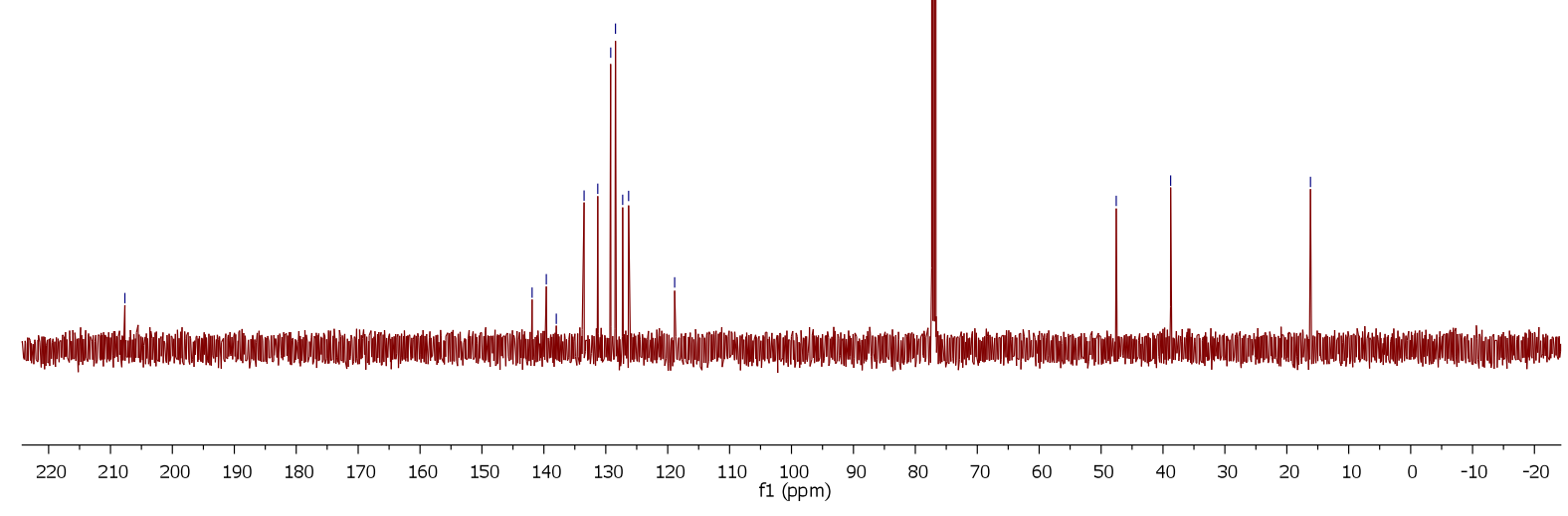

Figure S25: ${ }^{13} \mathrm{C}-\mathrm{NMR}$ of $1 \mathrm{j}$

S-35 


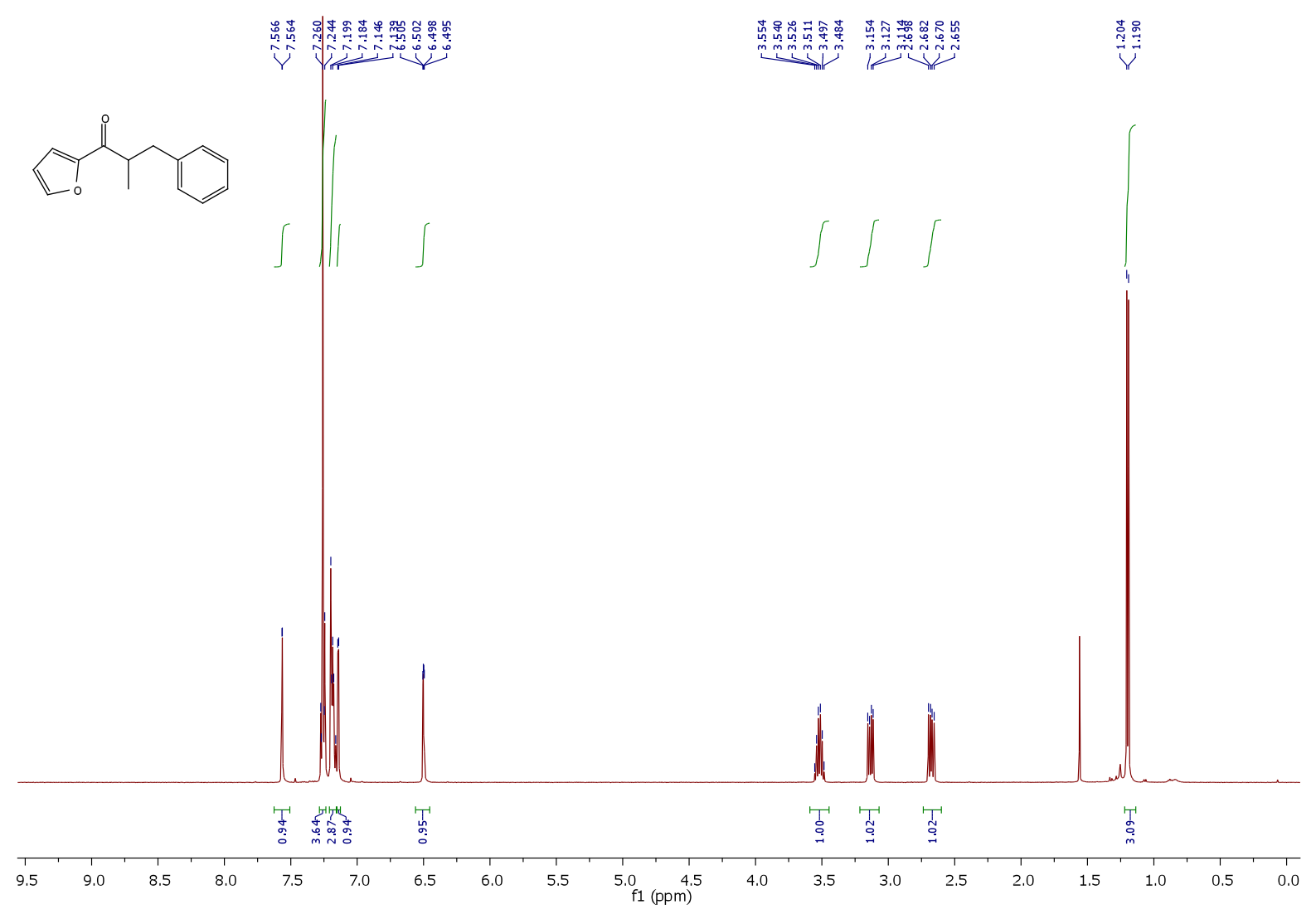

Figure S26: ${ }^{1} \mathrm{H}-\mathrm{NMR}$ of $1 \mathrm{k}$
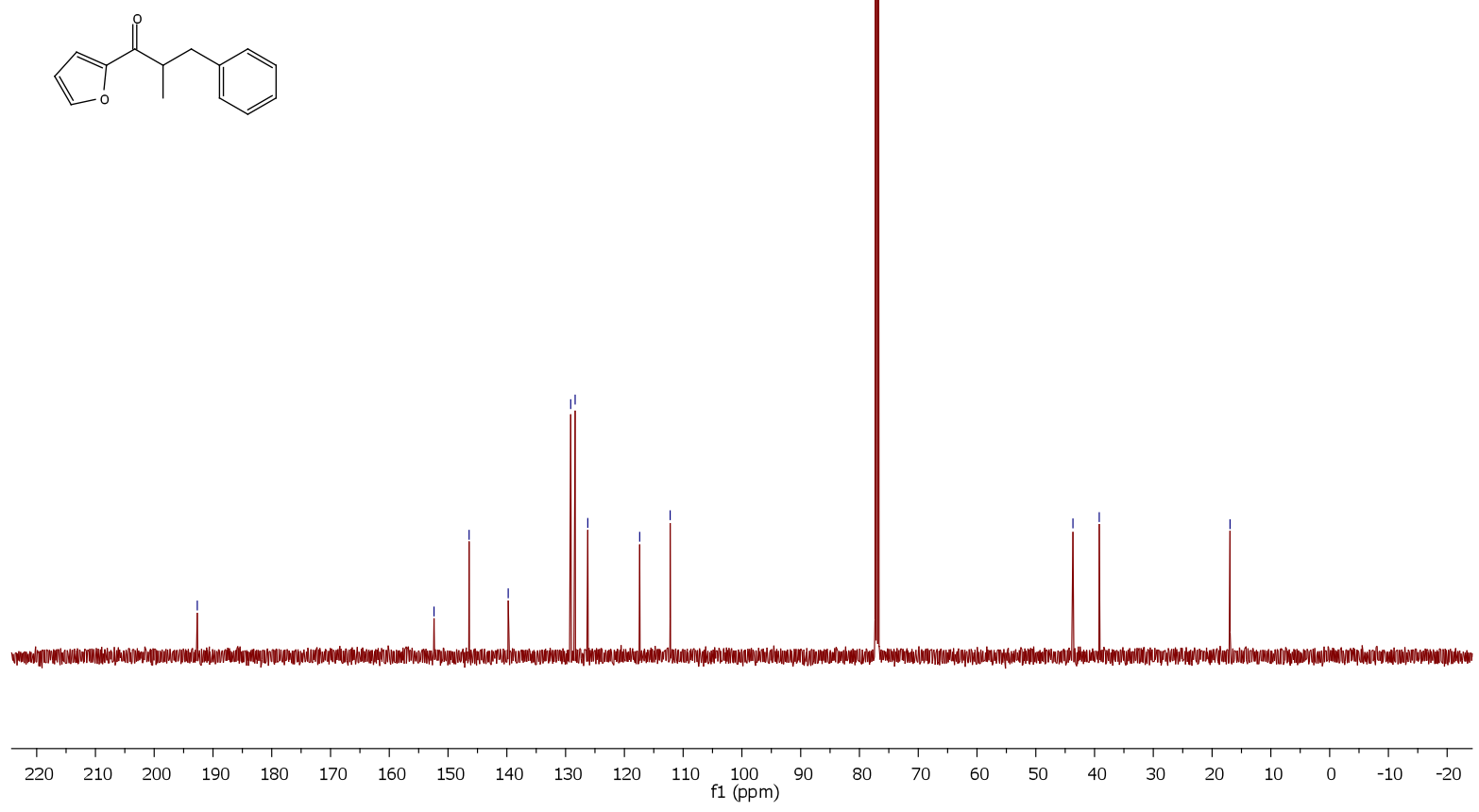

Figure S27: ${ }^{13} \mathrm{C}$-NMR of $1 \mathrm{k}$

S-36 


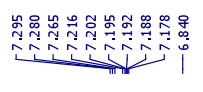
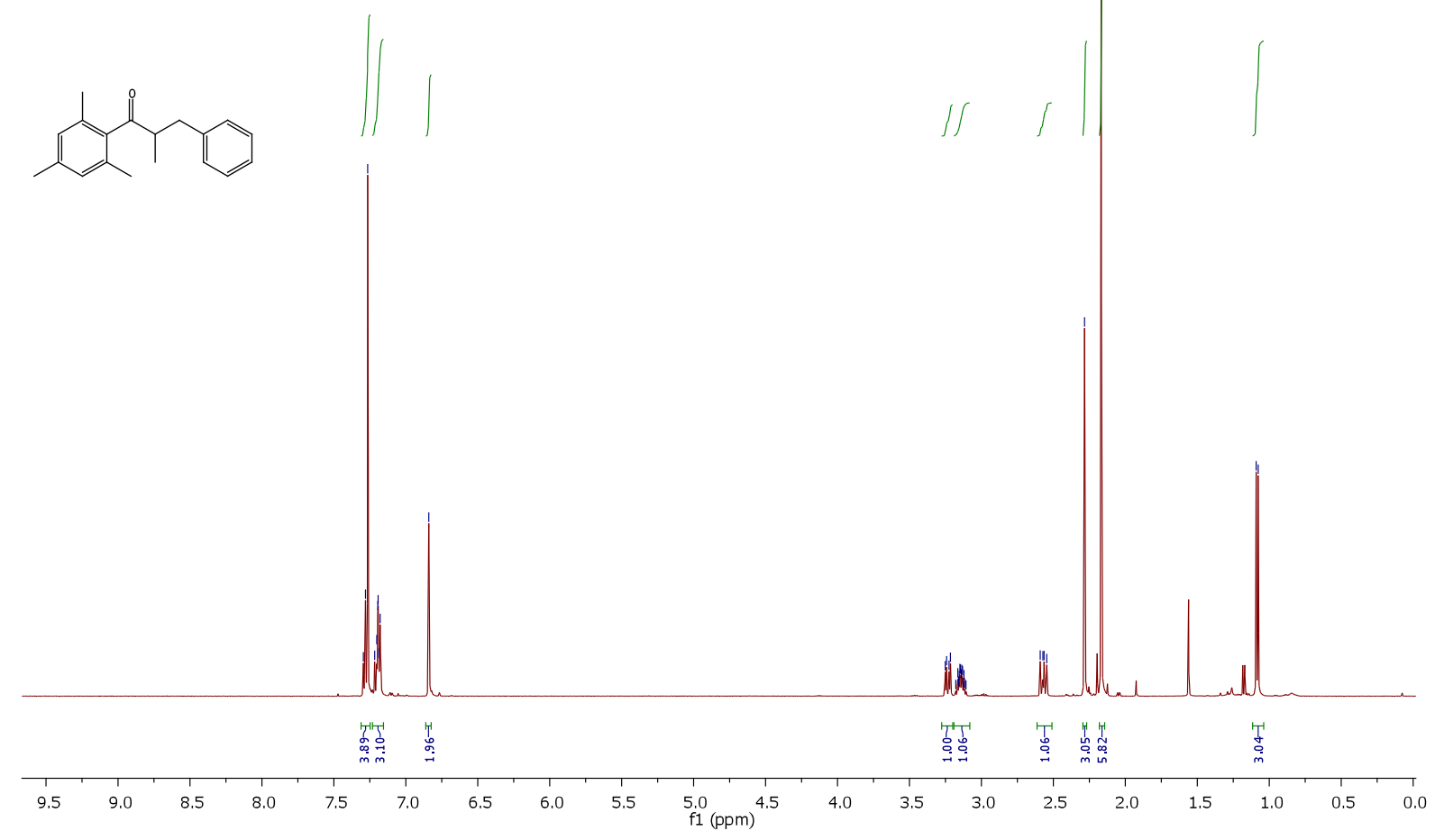

Figure S28: ${ }^{1} \mathrm{H}-\mathrm{NMR}$ of $1 \mathrm{I}$

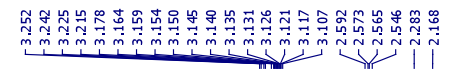

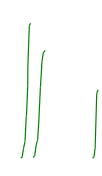

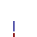

$\stackrel{\vec{a}}{\stackrel{\vec{m}}{\sim}}$

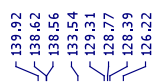
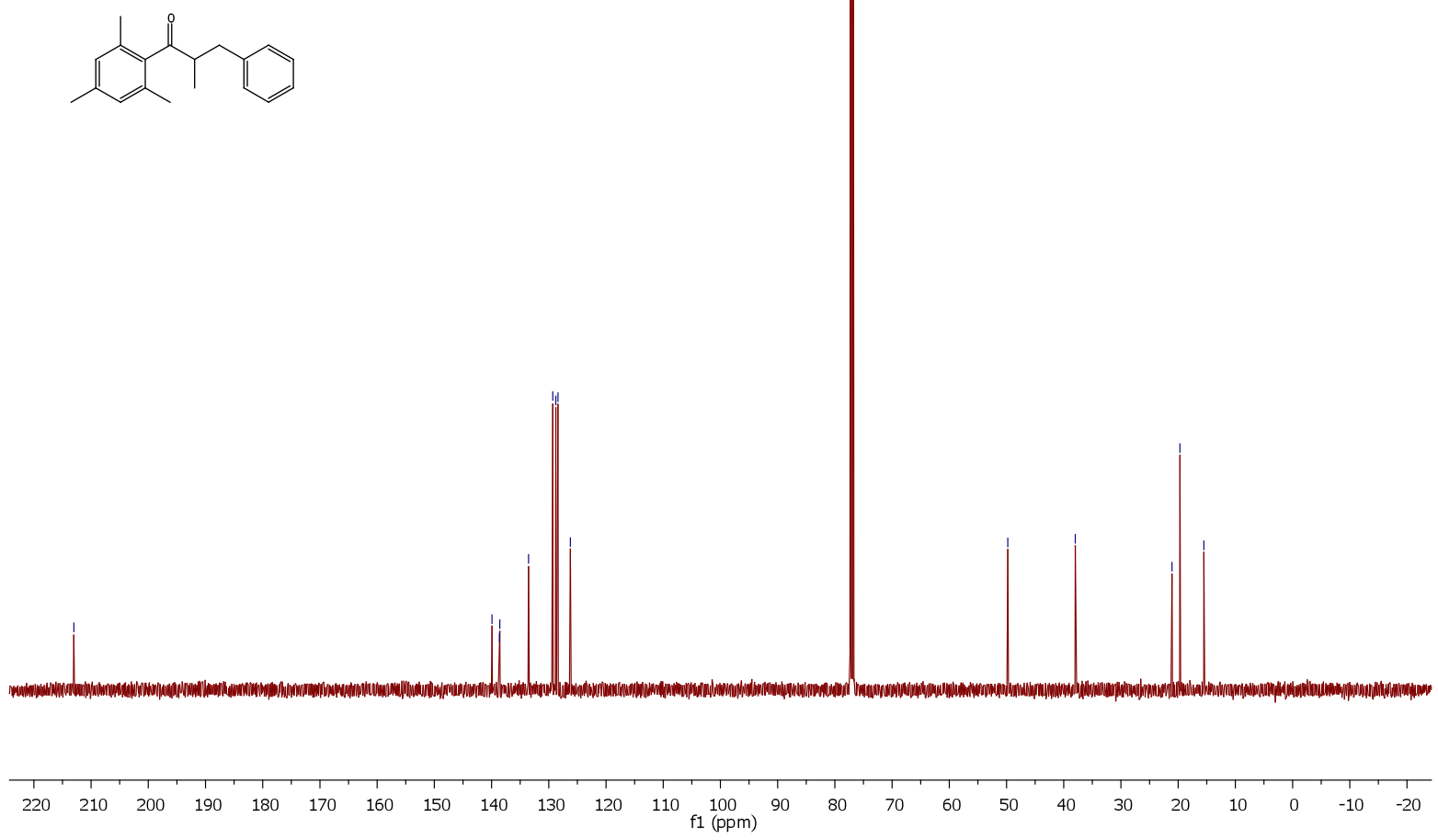

Figure S29: ${ }^{13} \mathrm{C}$-NMR of 1 I

S-37 


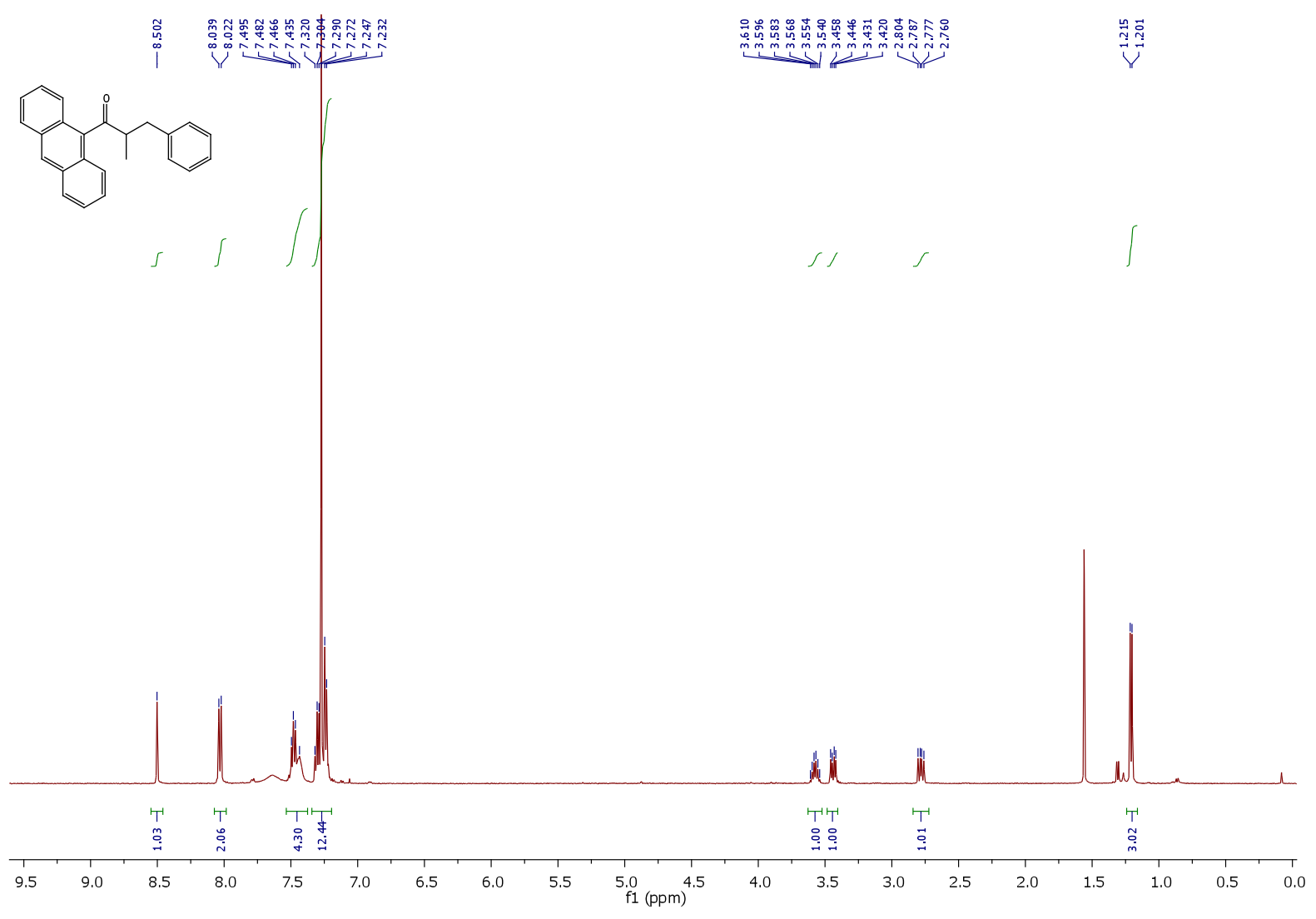

Figure S30: ${ }^{1} \mathrm{H}-\mathrm{NMR}$ of $1 \mathrm{~m}$

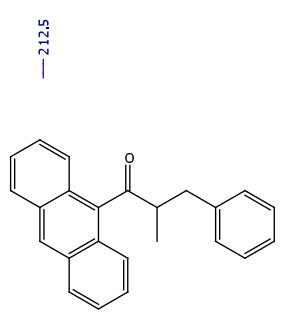

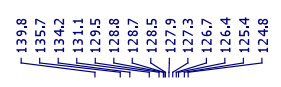

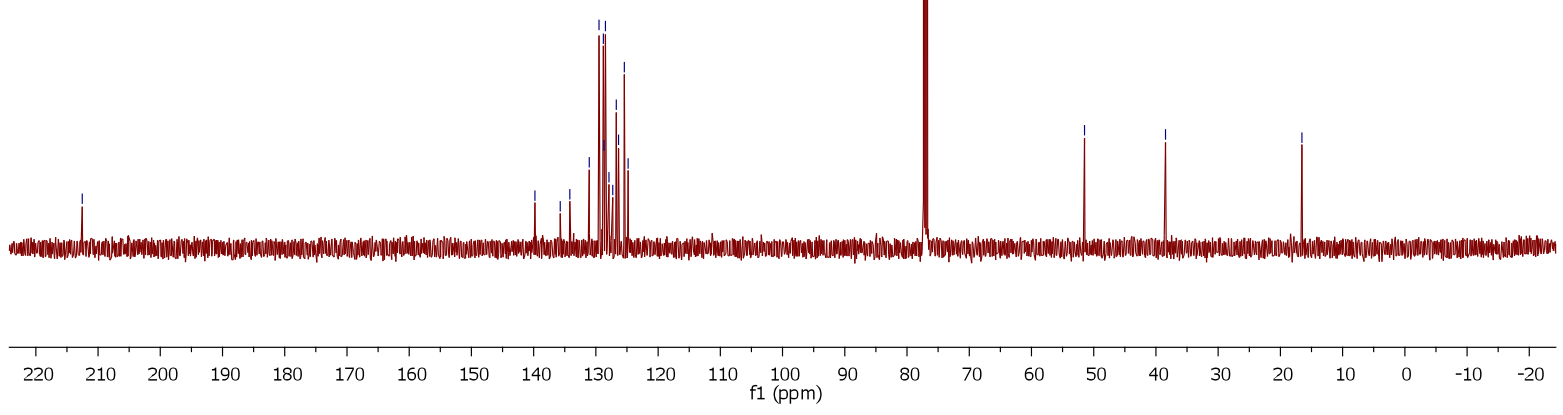

Figure S31: ${ }^{13} \mathrm{C}-\mathrm{NMR}$ of $1 \mathrm{~m}$

S-38 


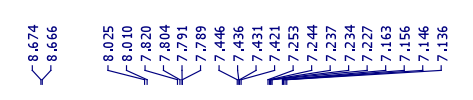
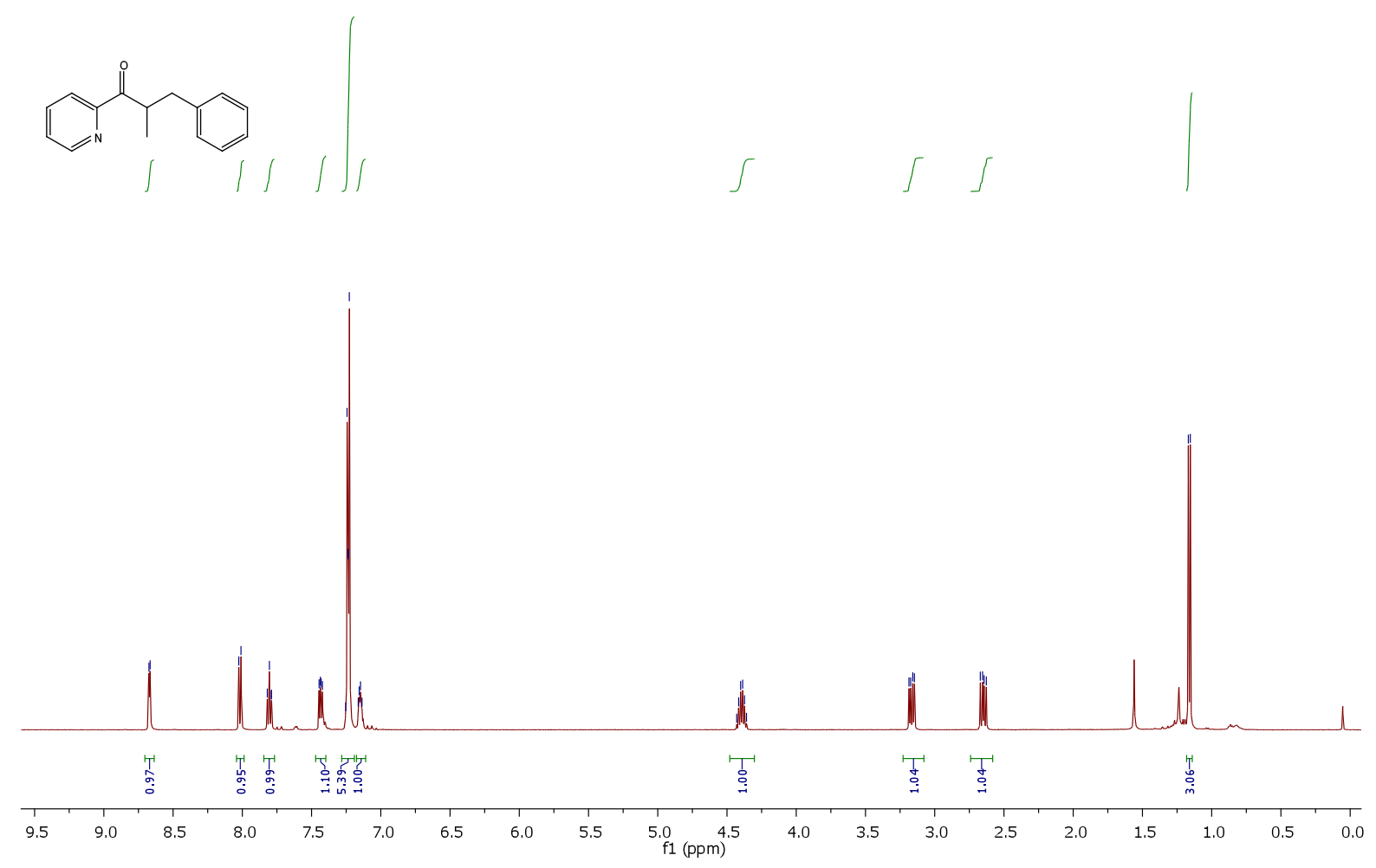

Figure S32: ${ }^{1} \mathrm{H}-\mathrm{NMR}$ of $1 \mathrm{n}$

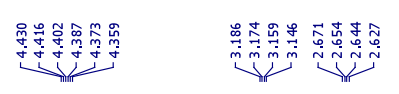

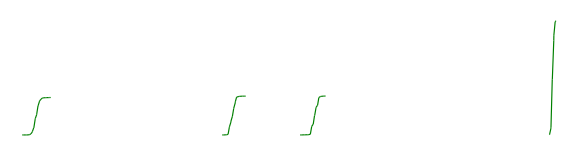

Y

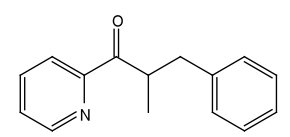

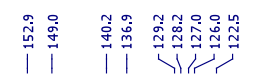

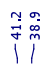

$\stackrel{0}{i}$

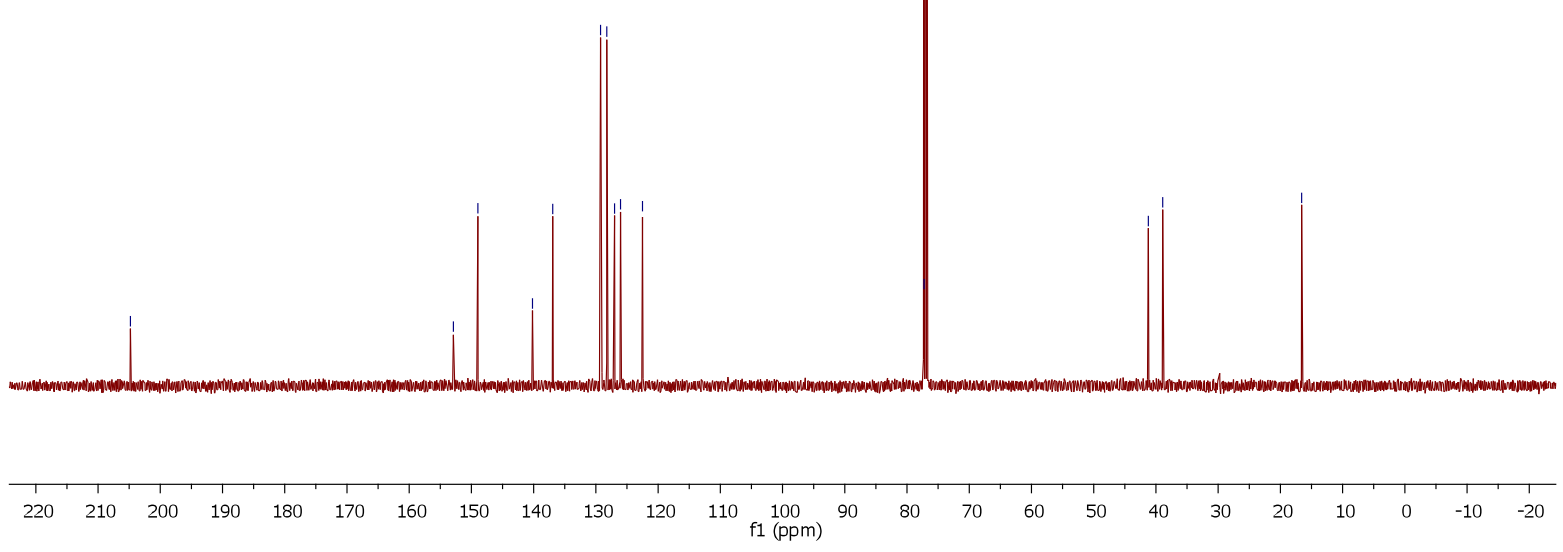

Figure S33: ${ }^{13} \mathrm{C}-\mathrm{NMR}$ of $1 \mathrm{n}$

S-39 


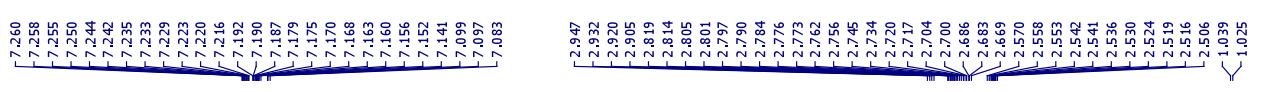
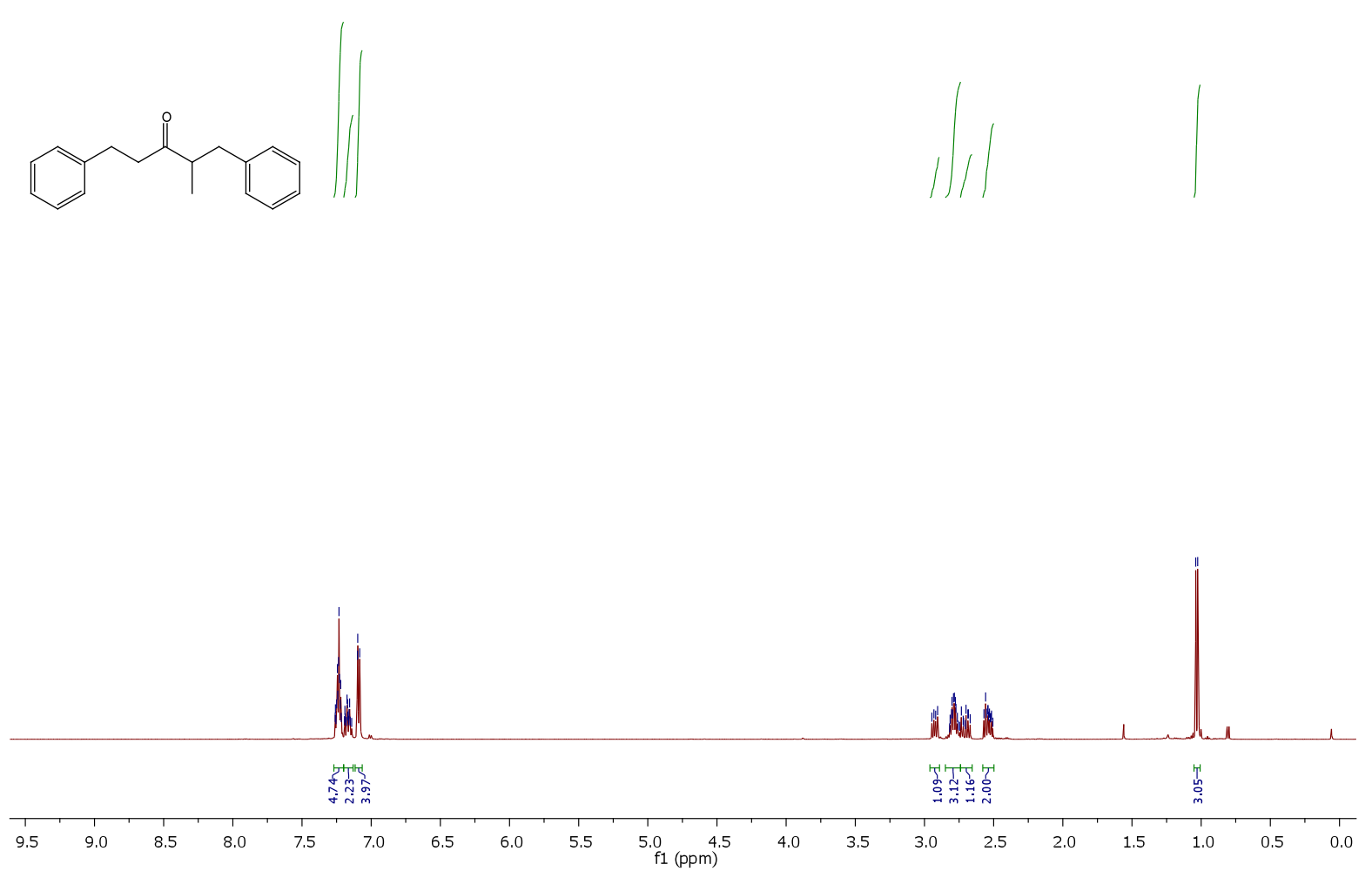

Figure S34: ${ }^{1} \mathrm{H}-\mathrm{NMR}$ of 10

i
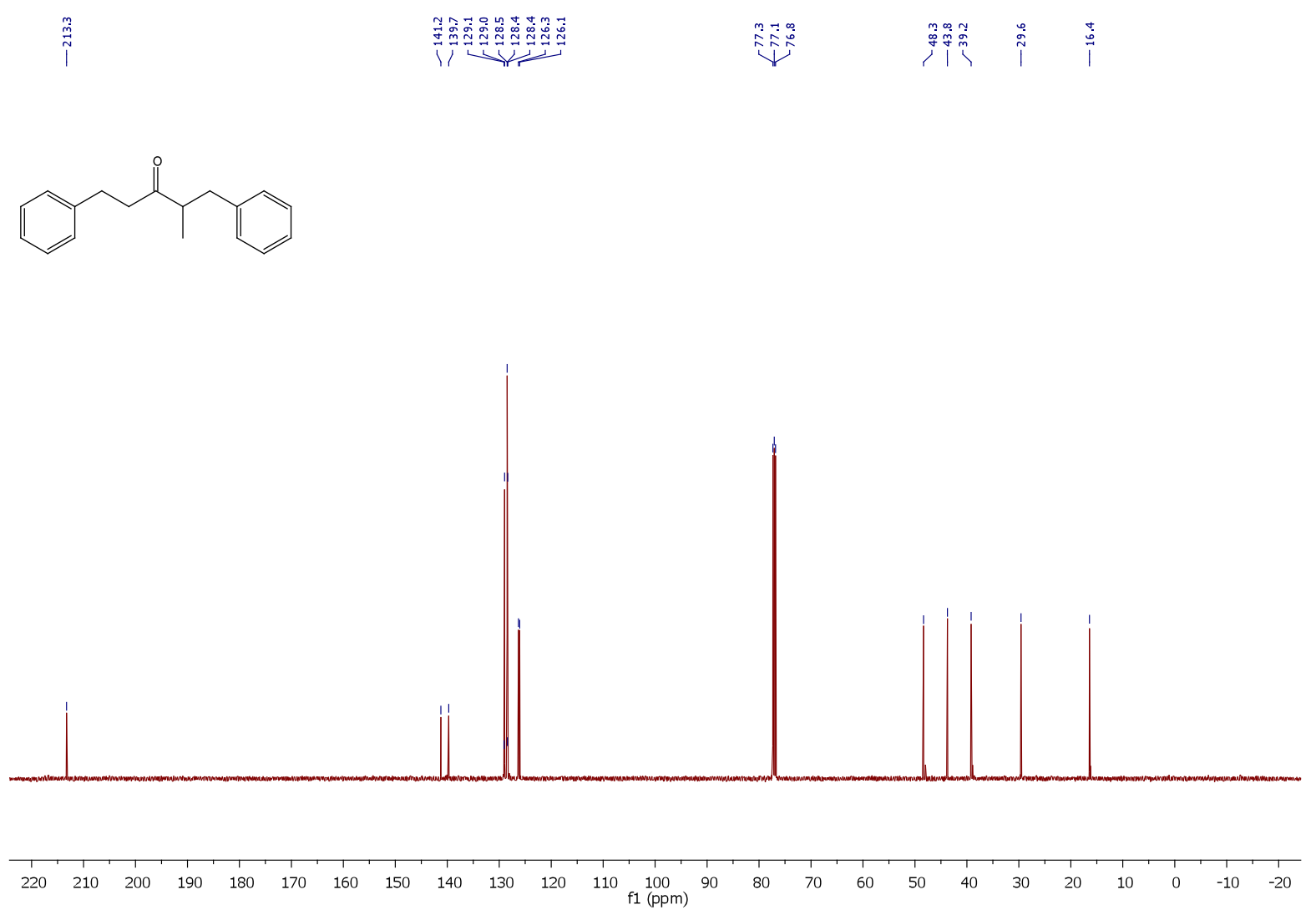

Figure S35: ${ }^{13} \mathrm{C}-\mathrm{NMR}$ of 10

S-40 


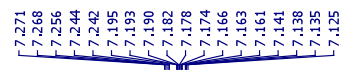

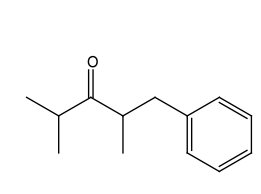

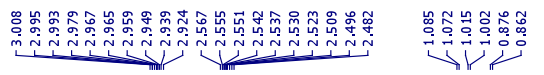

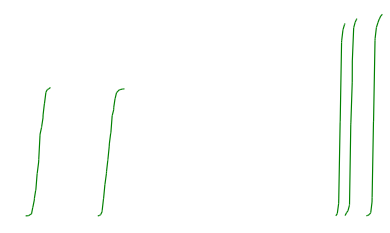

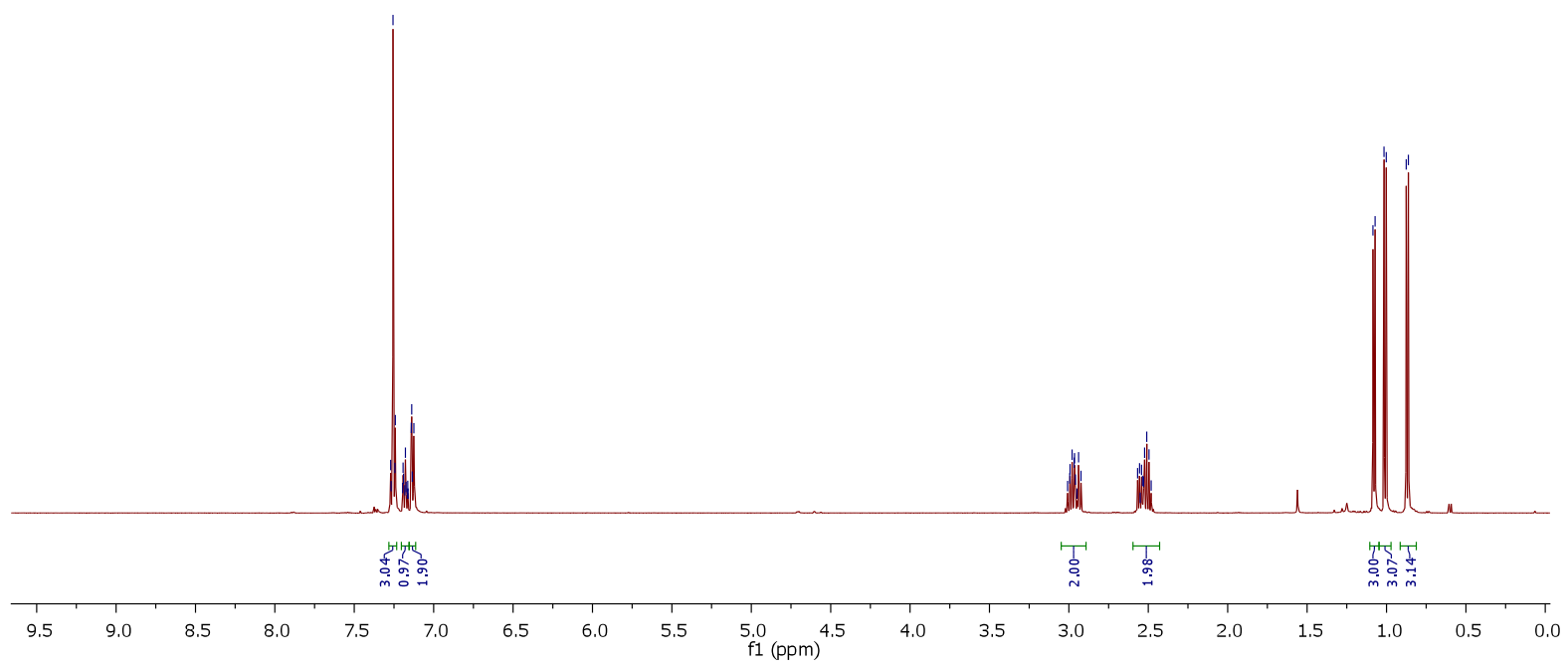

Figure S36: ${ }^{1} \mathrm{H}-\mathrm{NMR}$ of $1 \mathrm{p}$

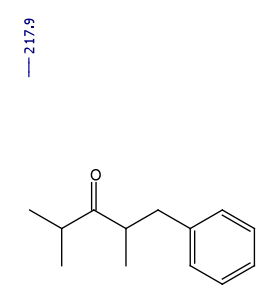

辛翌影

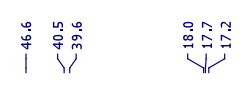
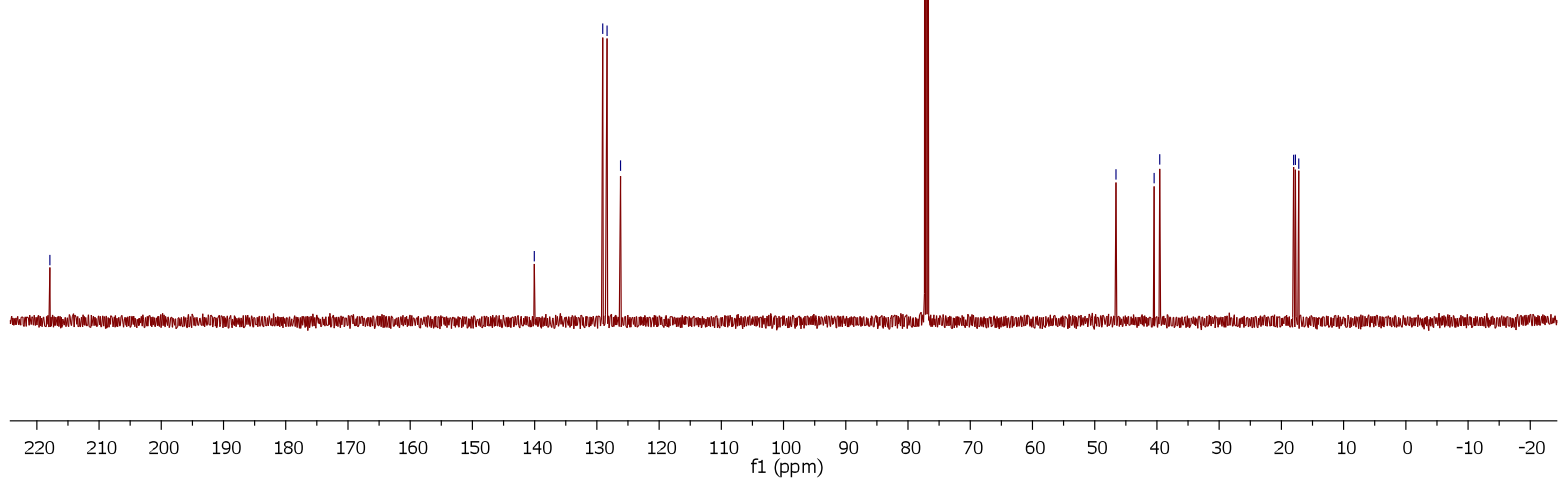

Figure S37: ${ }^{13} \mathrm{C}-\mathrm{NMR}$ of $1 \mathrm{p}$

S-41 

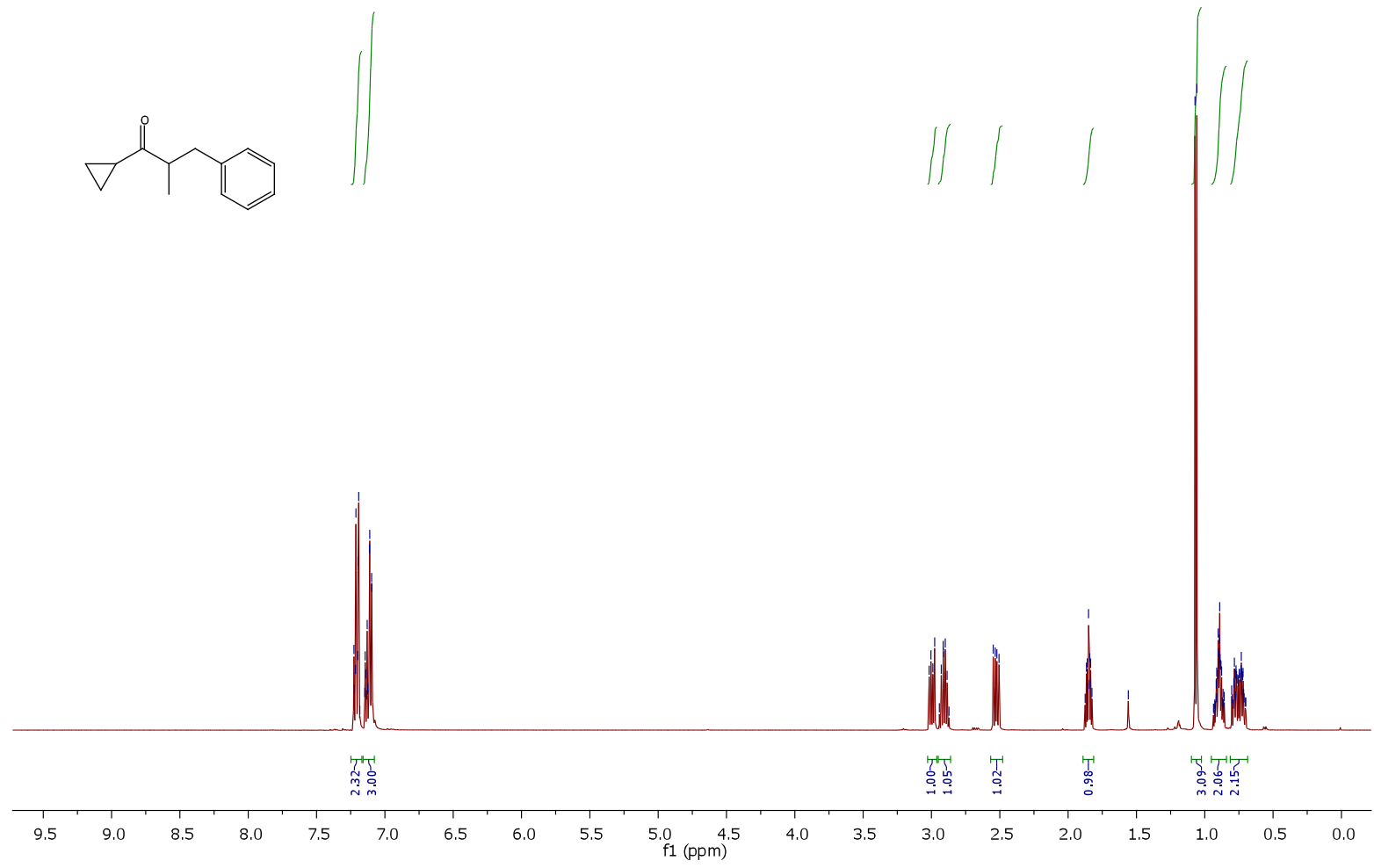

Figure S38: ${ }^{1} \mathrm{H}-\mathrm{NMR}$ of $1 \mathrm{q}$

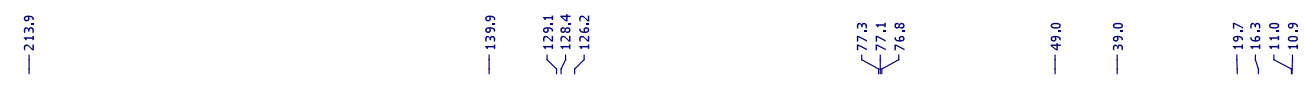
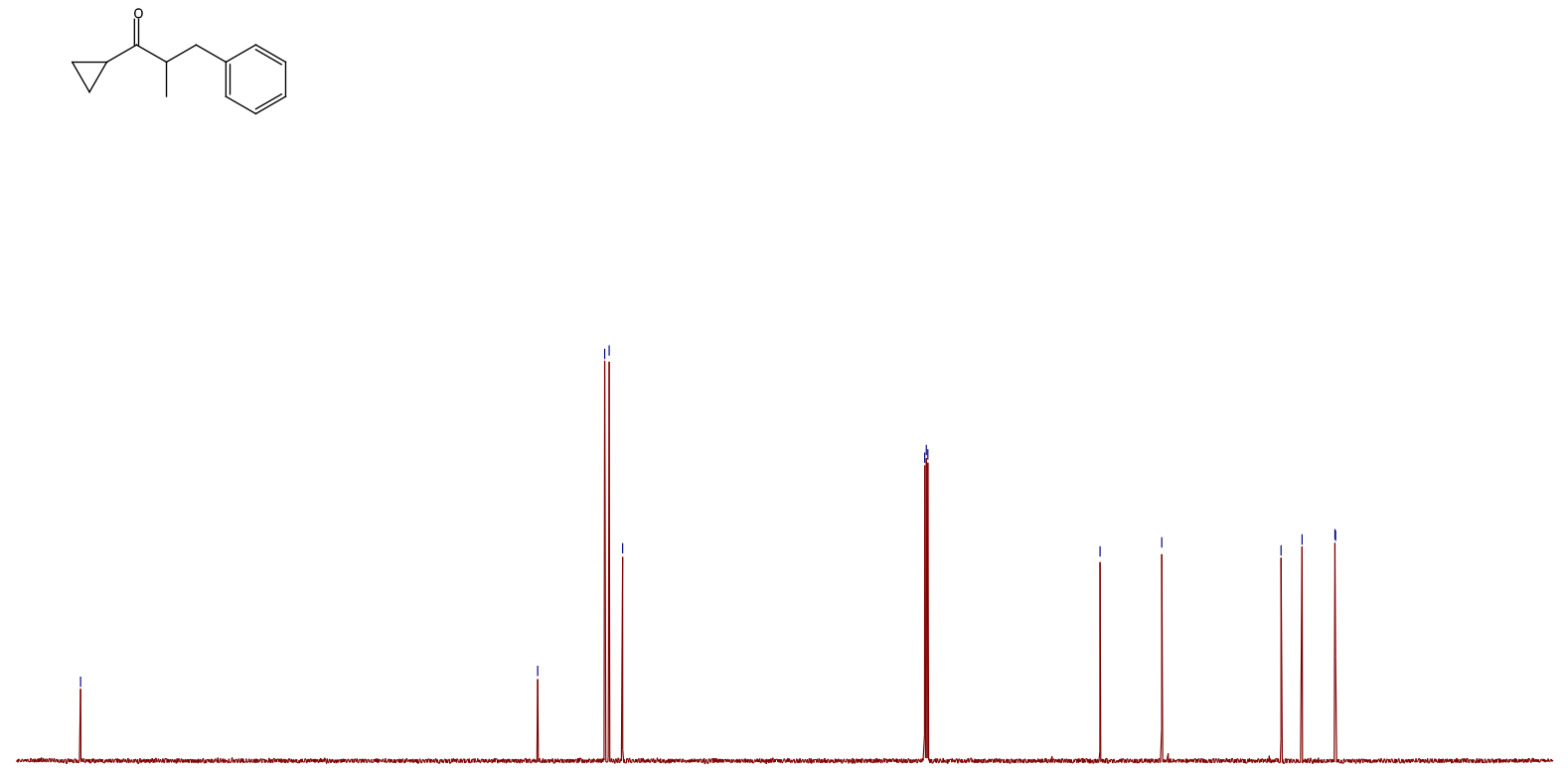

\begin{tabular}{llllllllllllllllllllllllll}
\hline 220 & 210 & 200 & 190 & 180 & 170 & 160 & 150 & 140 & 130 & 120 & 110 & 100 & 90 & 80 & 70 & 60 & 50 & 40 & 30 & 20 & 10 & 0 & -10 & -20
\end{tabular}

Figure S39: ${ }^{13} \mathrm{C}-\mathrm{NMR}$ of $1 \mathrm{q}$ 


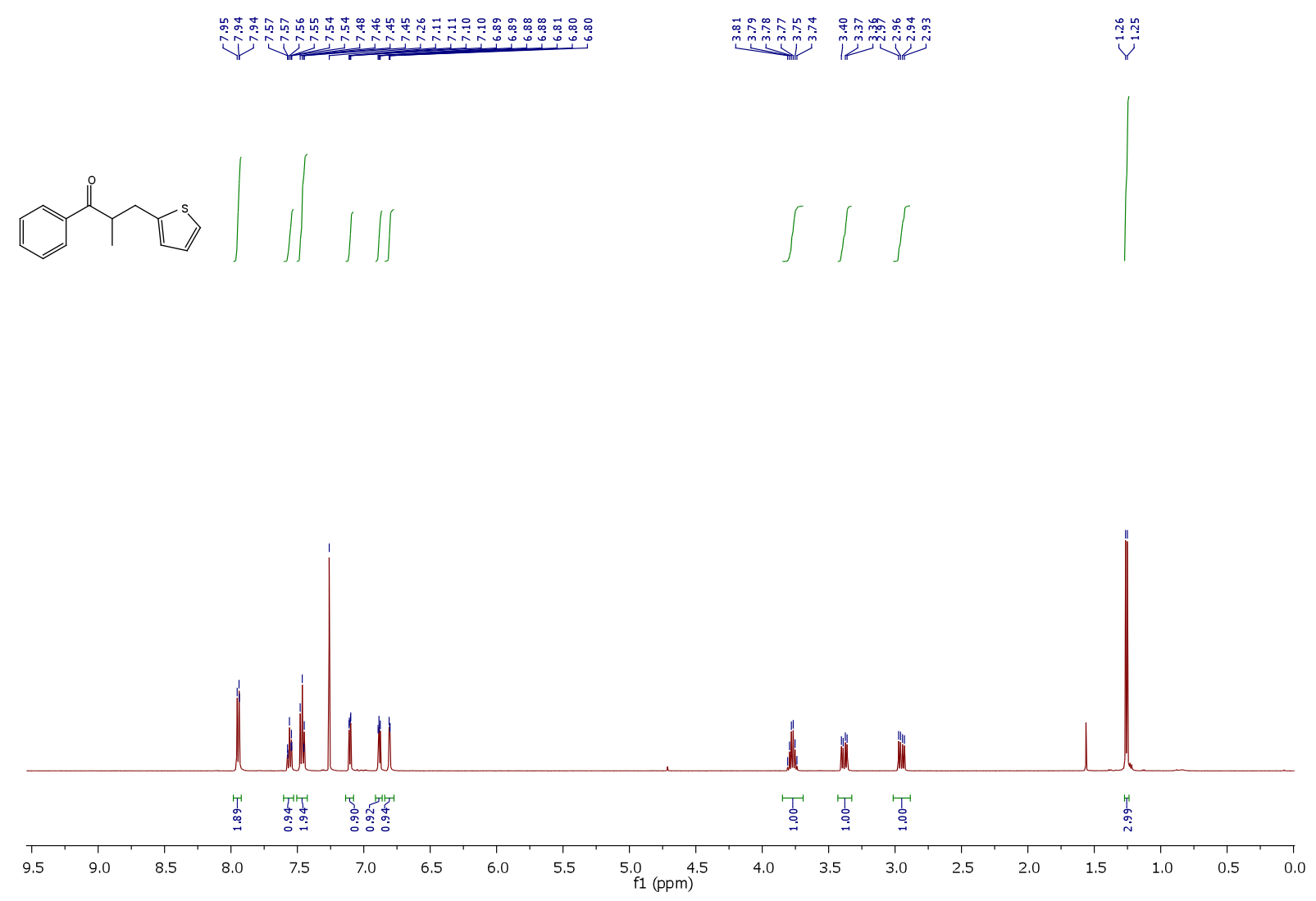

Figure S40: ${ }^{1} \mathrm{H}-\mathrm{NMR}$ of $1 \mathrm{r}$

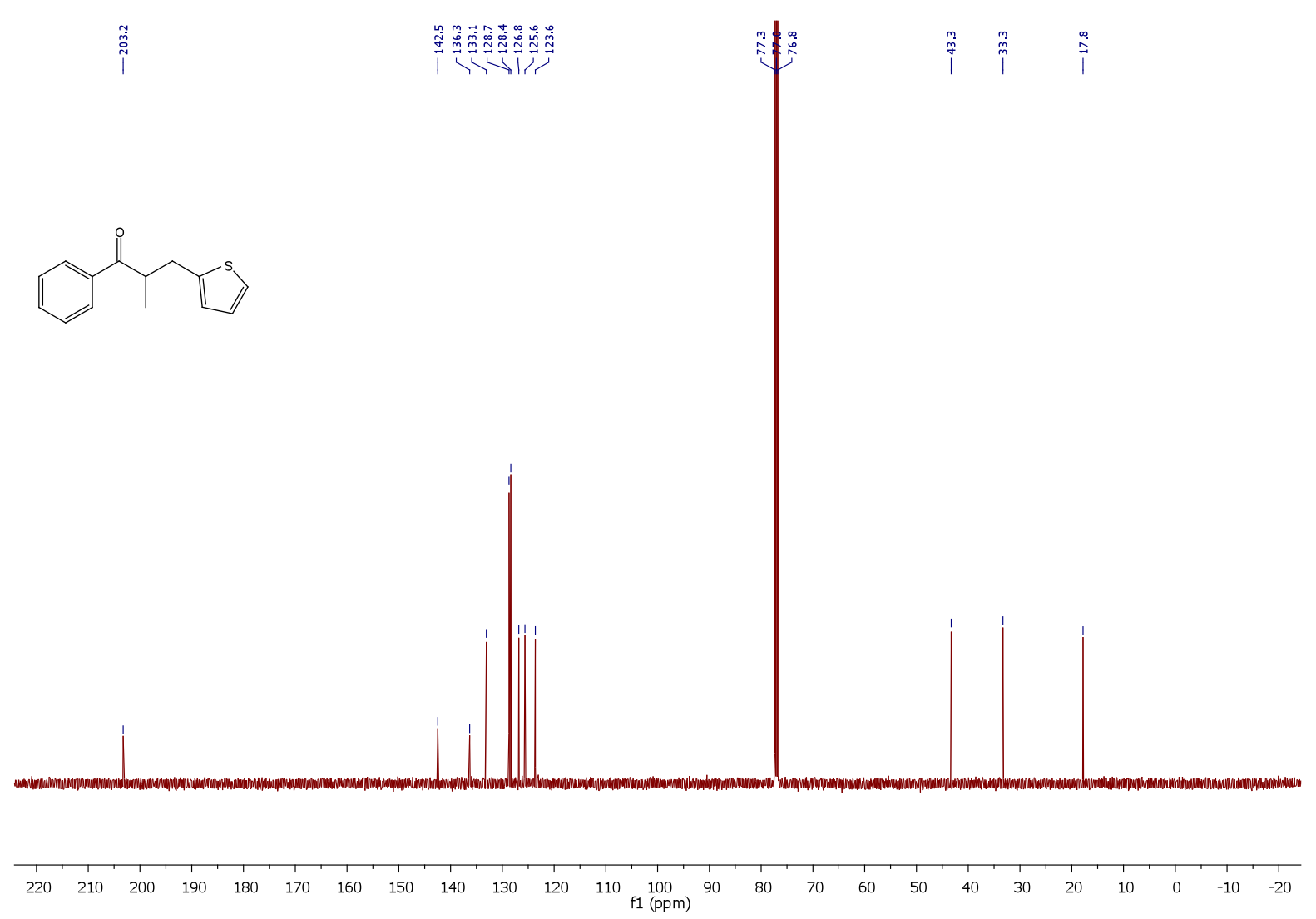

Figure S41: ${ }^{13} \mathrm{C}-\mathrm{NMR}$ of $1 \mathrm{r}$ 


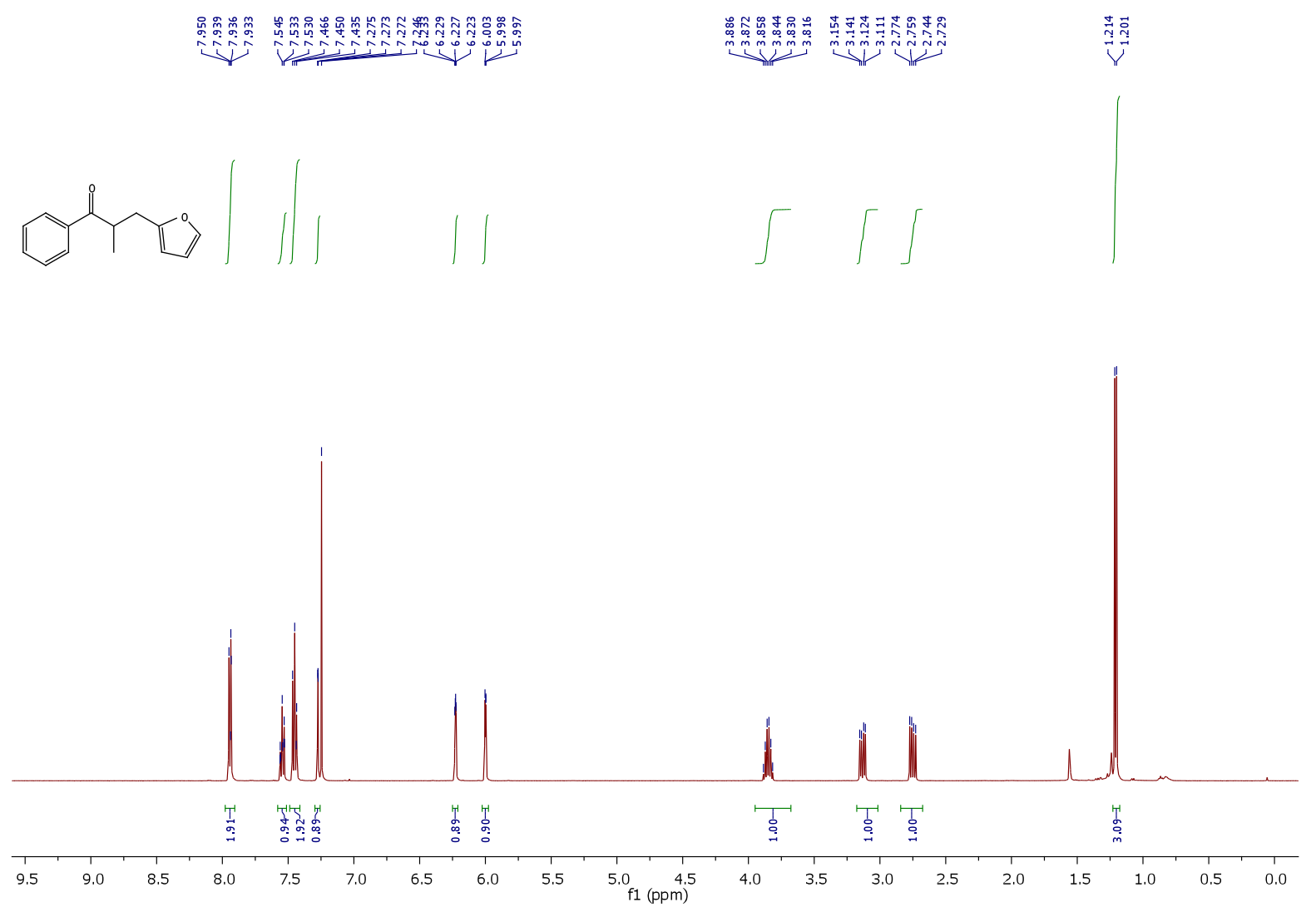

Figure S42: ${ }^{1} \mathrm{H}-\mathrm{NMR}$ of $1 \mathrm{~s}$

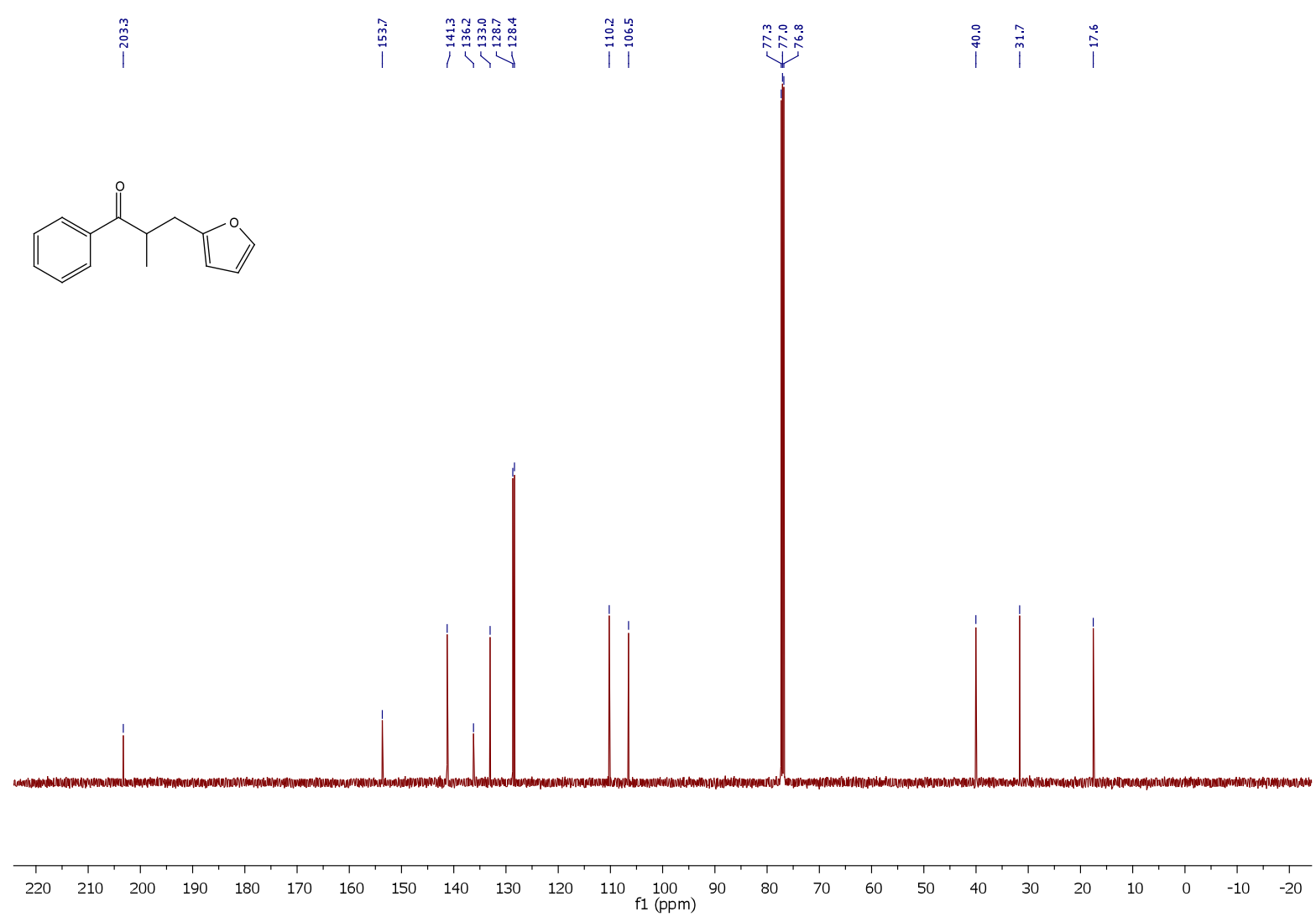

Figure S43: ${ }^{13} \mathrm{C}$-NMR of $1 \mathrm{~s}$ 


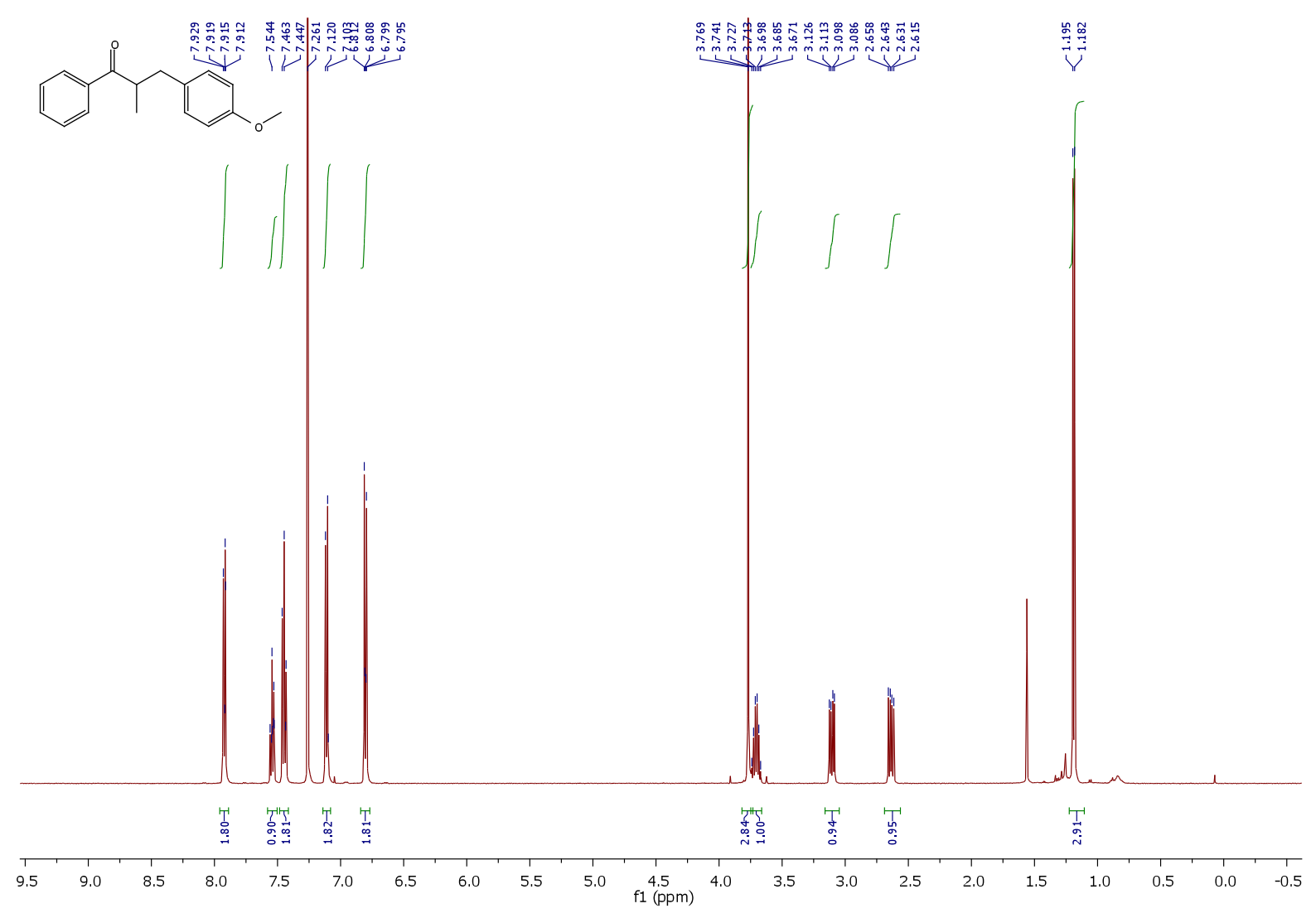

Figure S44: ${ }^{1} \mathrm{H}-\mathrm{NMR}$ of $1 \mathrm{t}$

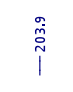

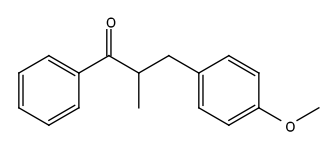

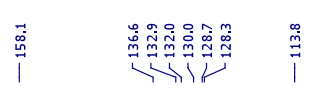

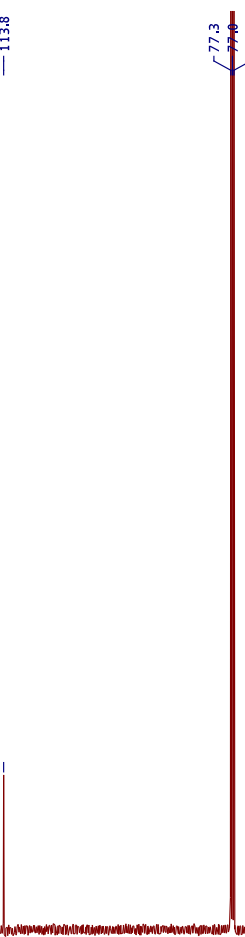

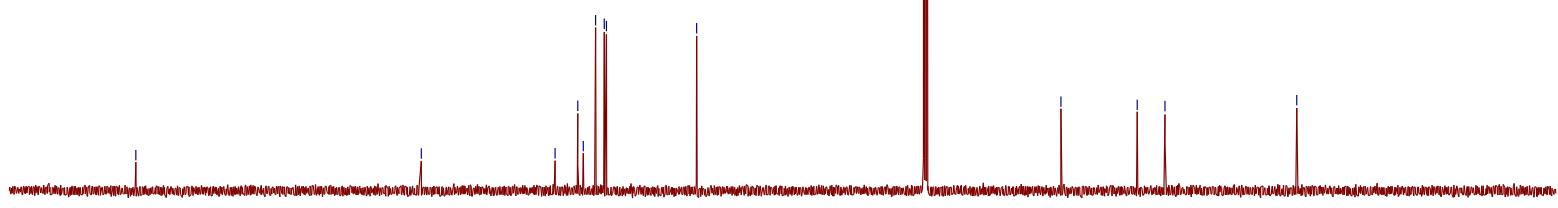

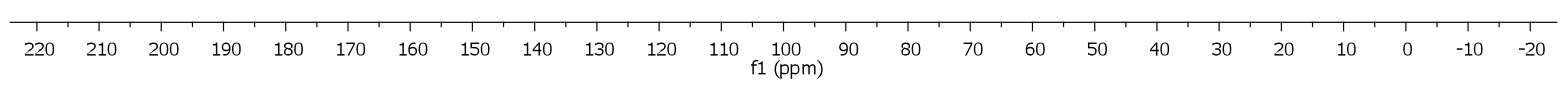

Figure S45: ${ }^{13} \mathrm{C}-\mathrm{NMR}$ of $1 \mathrm{t}$

S-45 


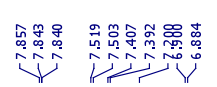

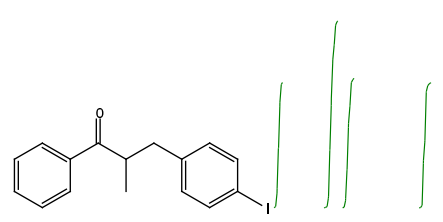

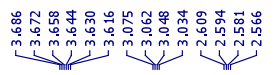

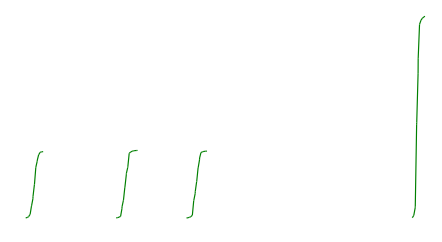

最

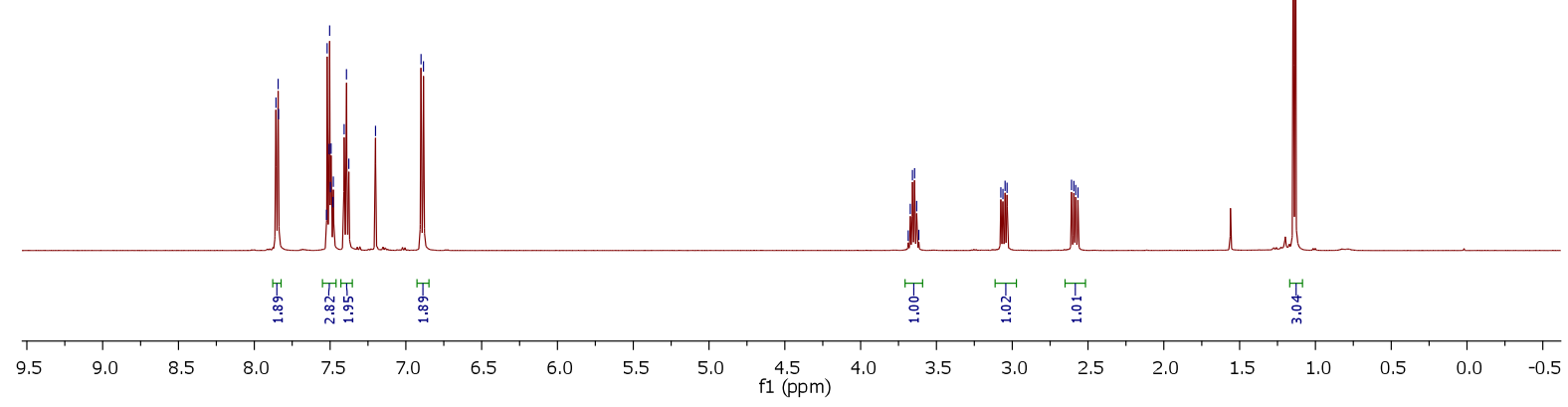

Figure S46: ${ }^{1} \mathrm{H}-\mathrm{NMR}$ of $1 \mathrm{u}$

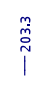

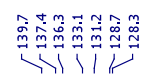

美

䇏

$\hat{i}$
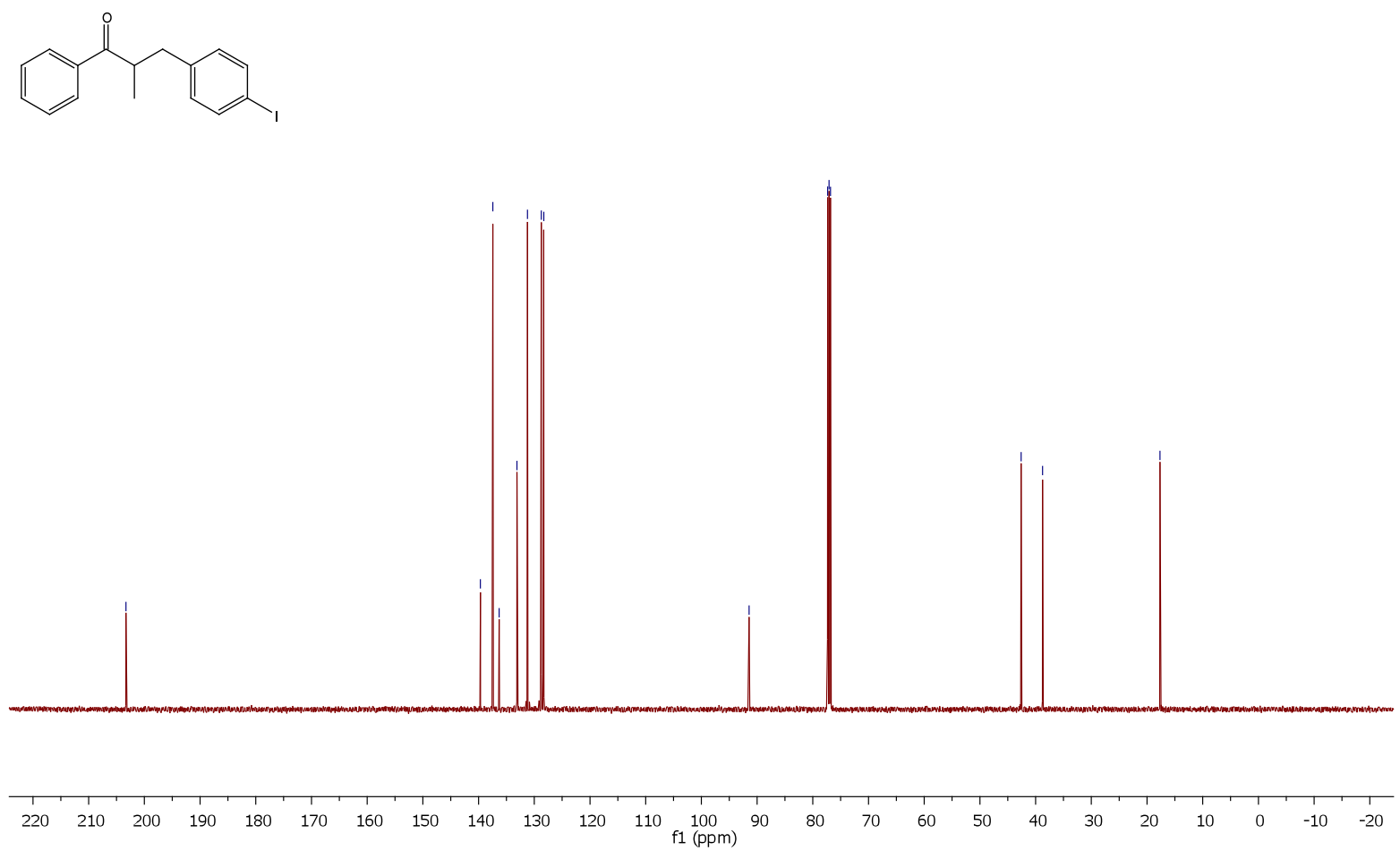

Figure S47: ${ }^{13} \mathrm{C}-\mathrm{NMR}$ of $1 \mathrm{u}$

S-46 


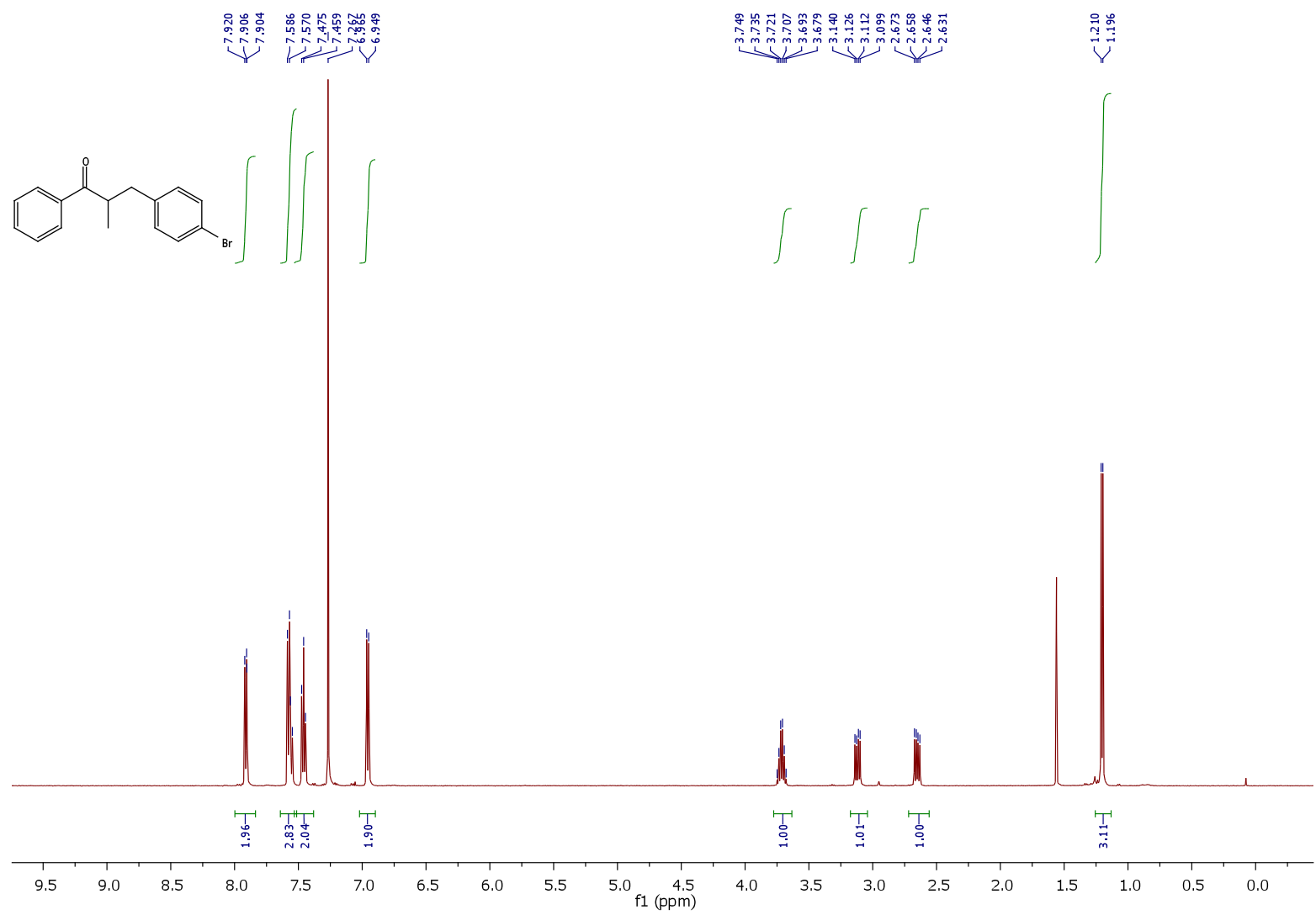

Figure S48: ${ }^{1} \mathrm{H}-\mathrm{NMR}$ of $1 \mathrm{v}$

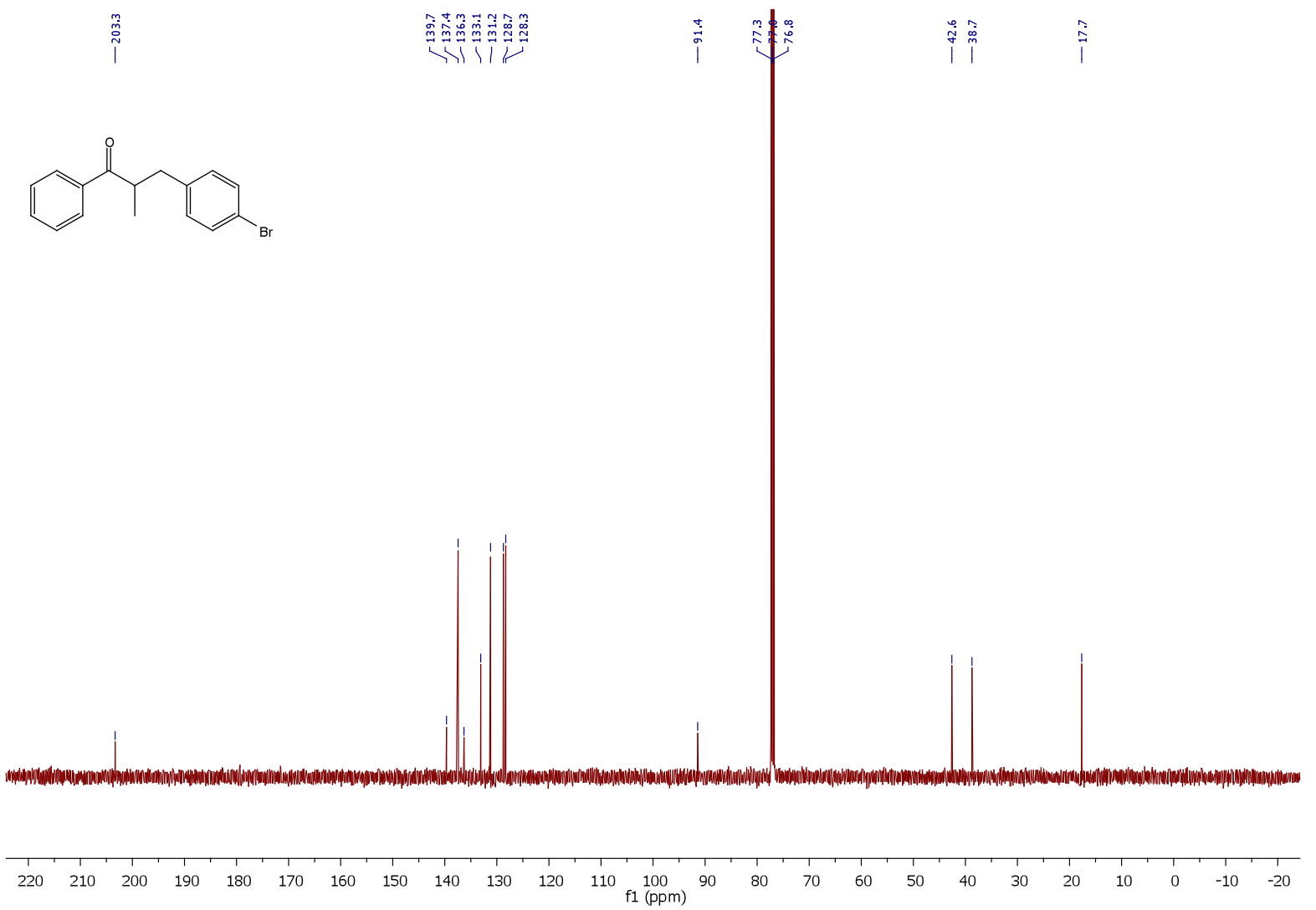

Figure S49: ${ }^{13} \mathrm{C}-\mathrm{NMR}$ of $1 \mathrm{v}$ 


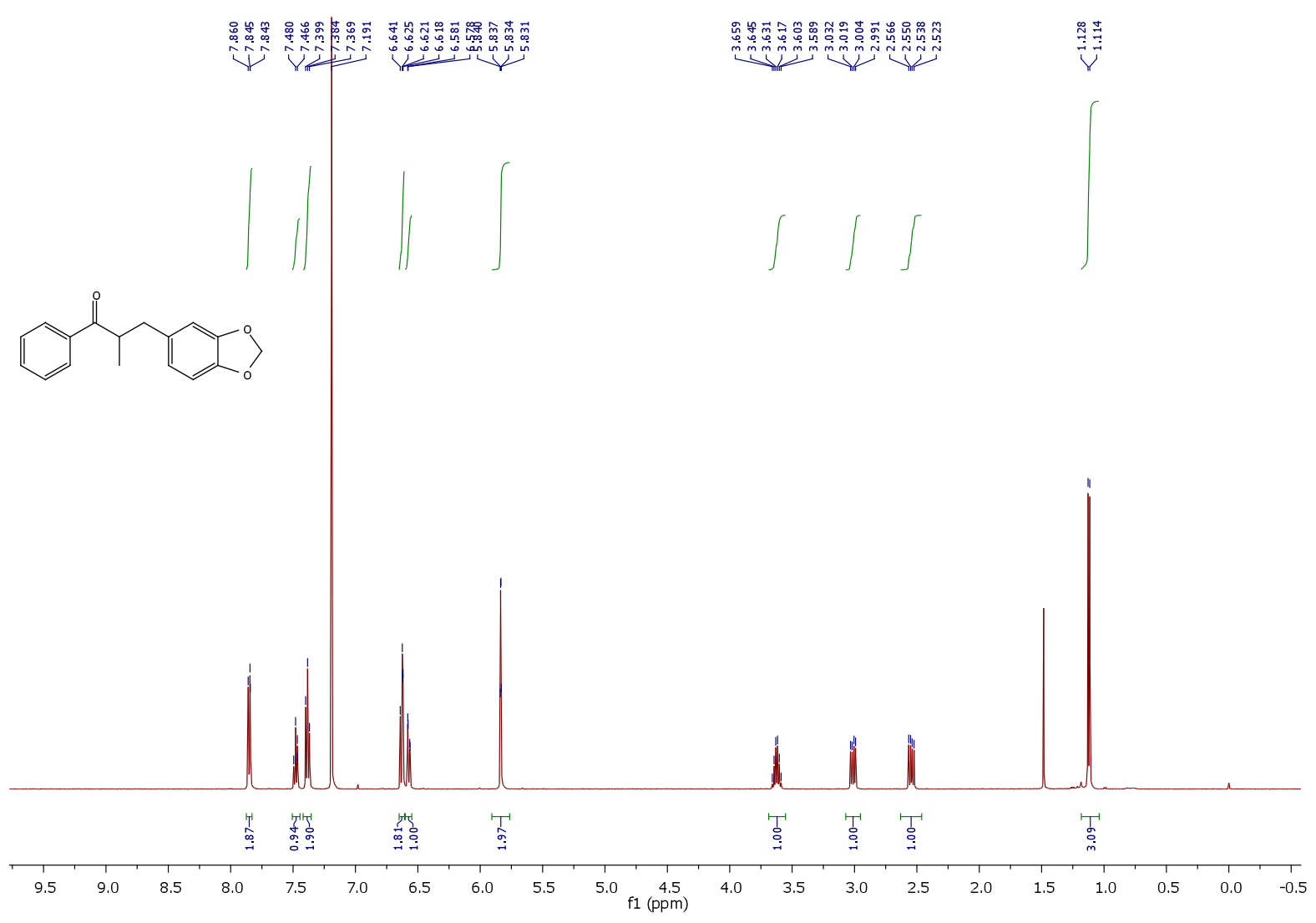

Figure S50: ${ }^{1} \mathrm{H}-\mathrm{NMR}$ of $1 \mathrm{v}$

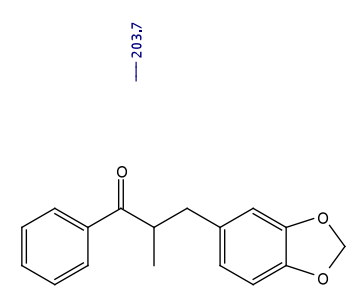

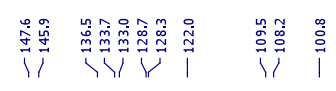

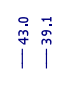

$\stackrel{+}{i}$

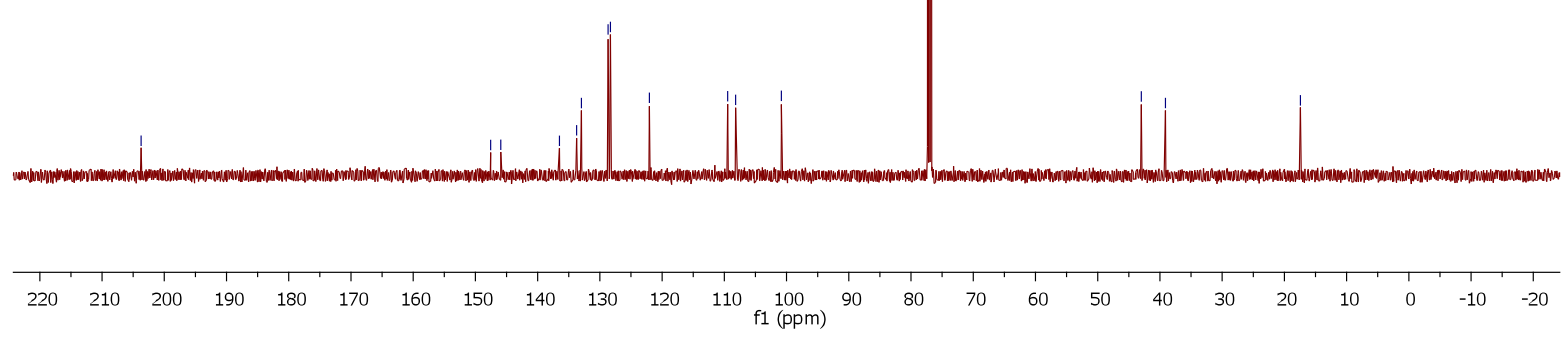

Figure S51: ${ }^{13} \mathrm{C}-\mathrm{NMR}$ of $1 \mathrm{w}$

S-48 


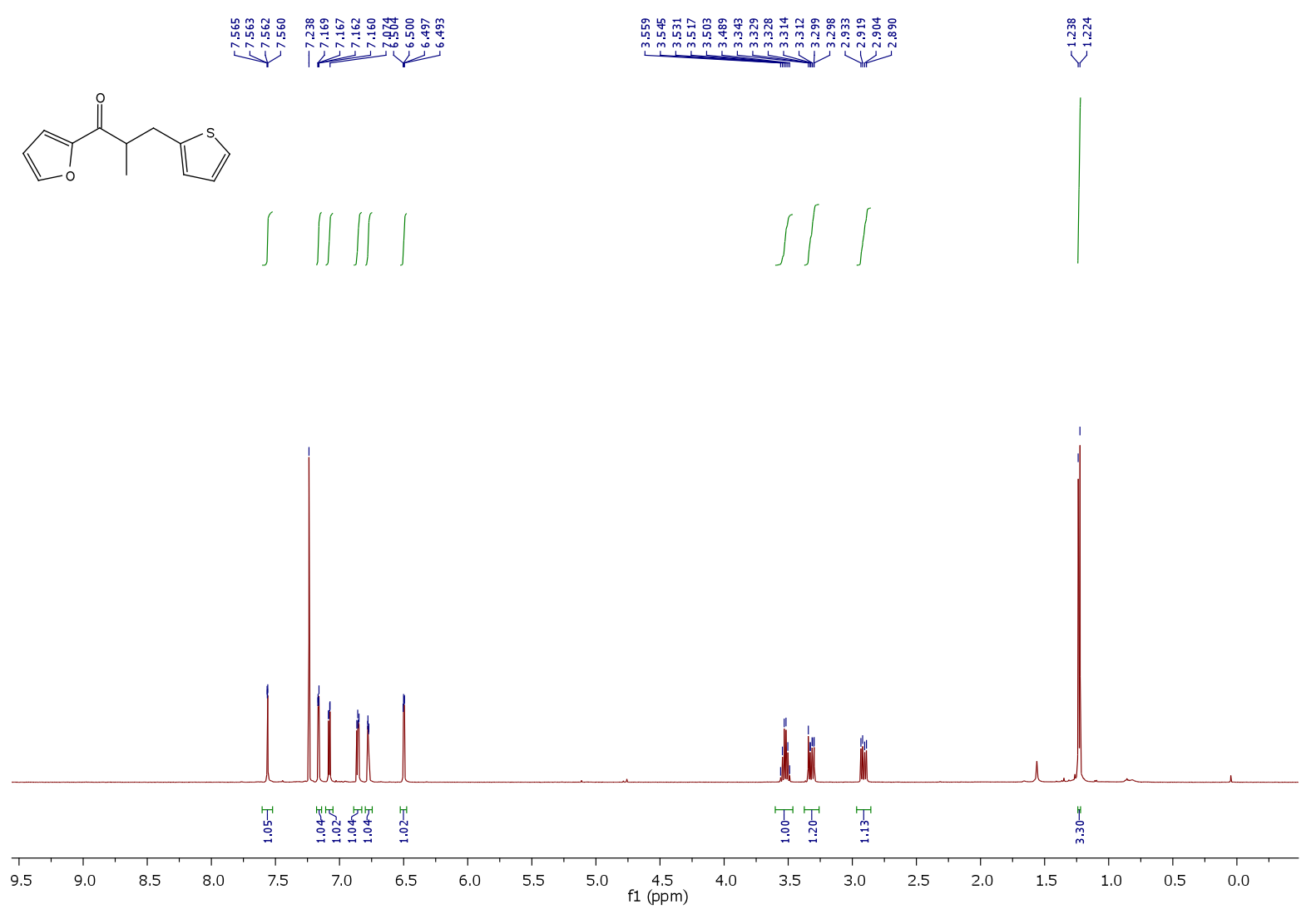

Figure S52: ${ }^{1} \mathrm{H}-\mathrm{NMR}$ of $1 \mathrm{x}$

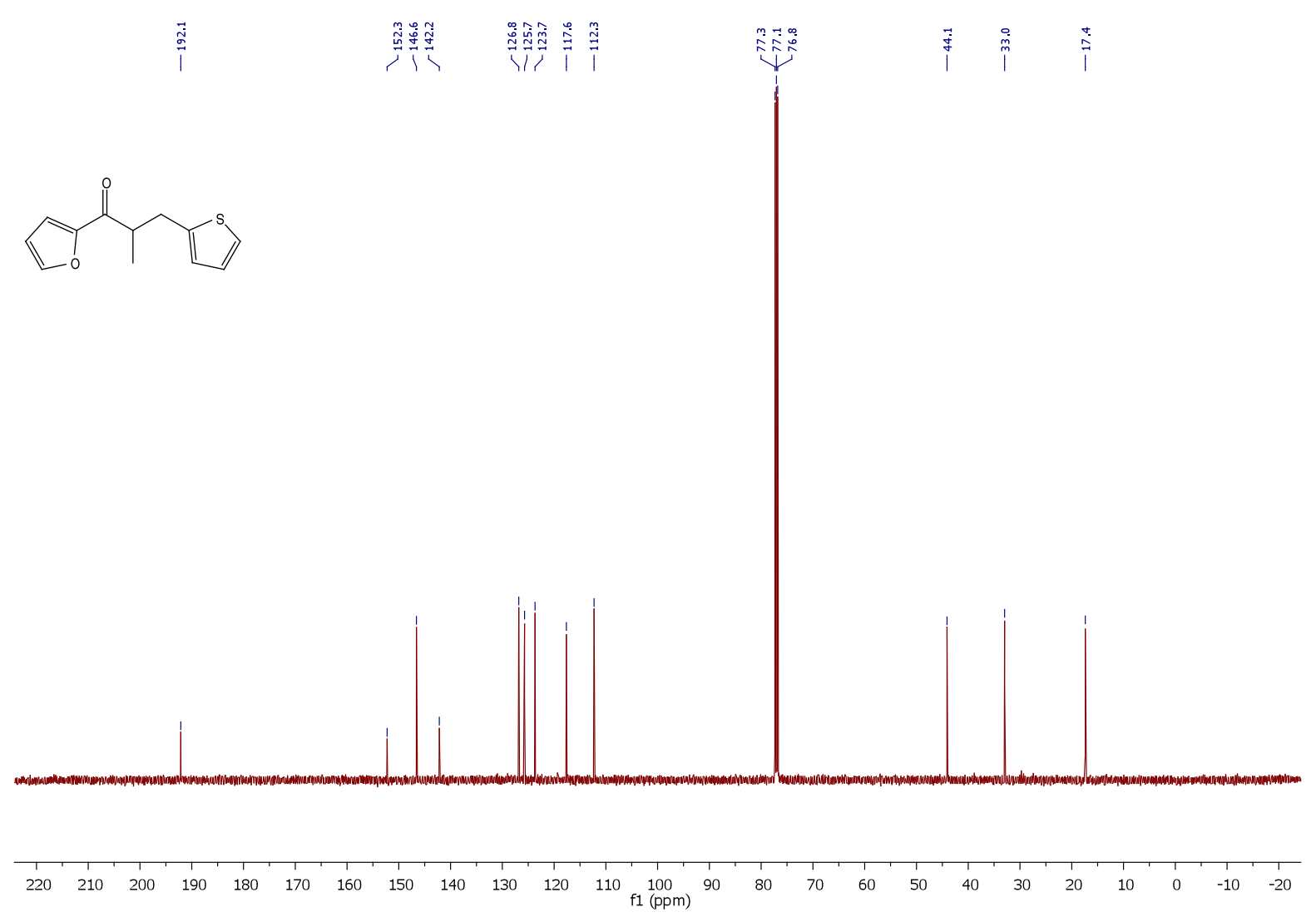

Figure S53: ${ }^{13} \mathrm{C}-\mathrm{NMR}$ of $1 \mathrm{x}$ 


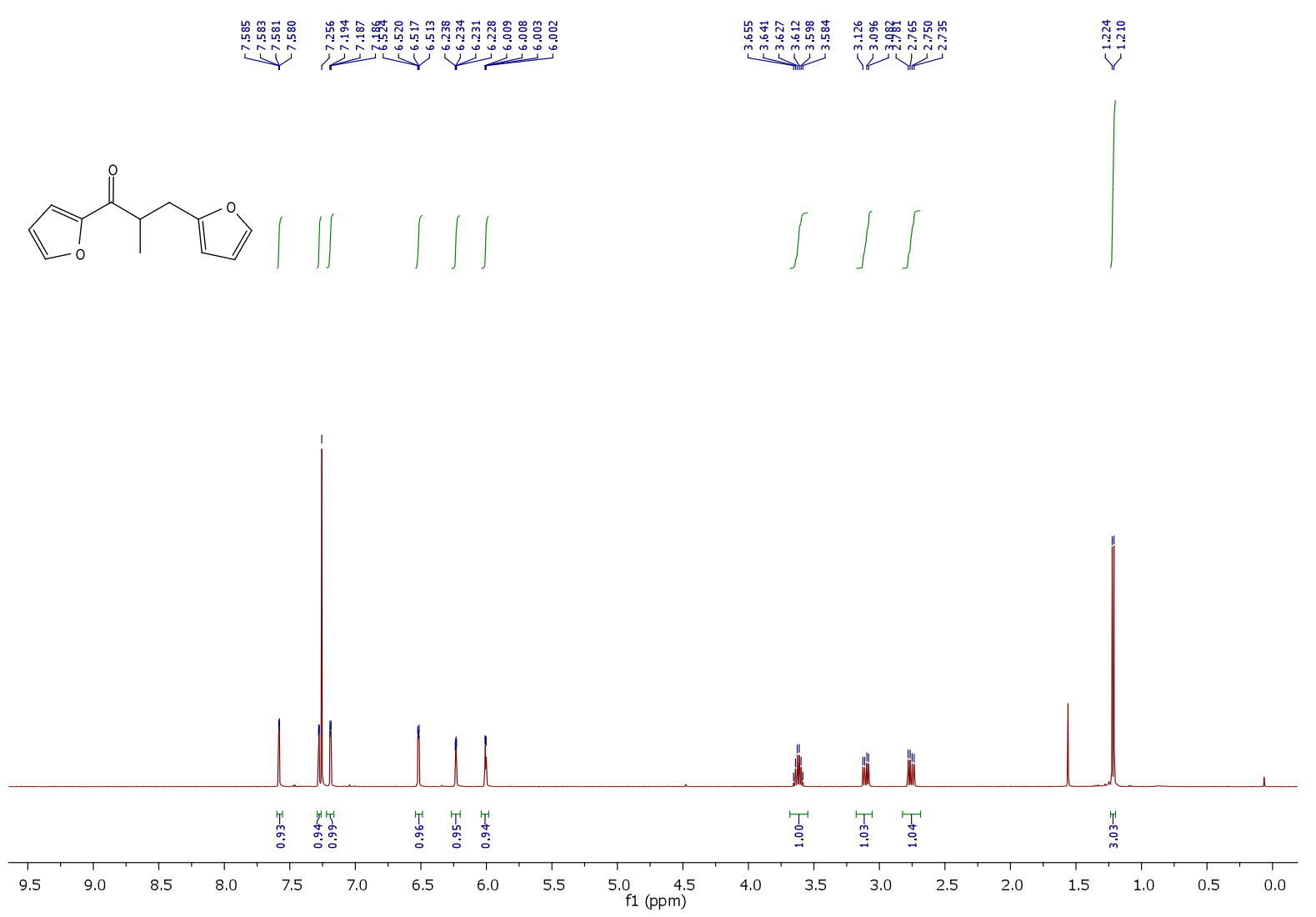

Figure S54: ${ }^{1} \mathrm{H}-\mathrm{NMR}$ of $1 \mathrm{y}$

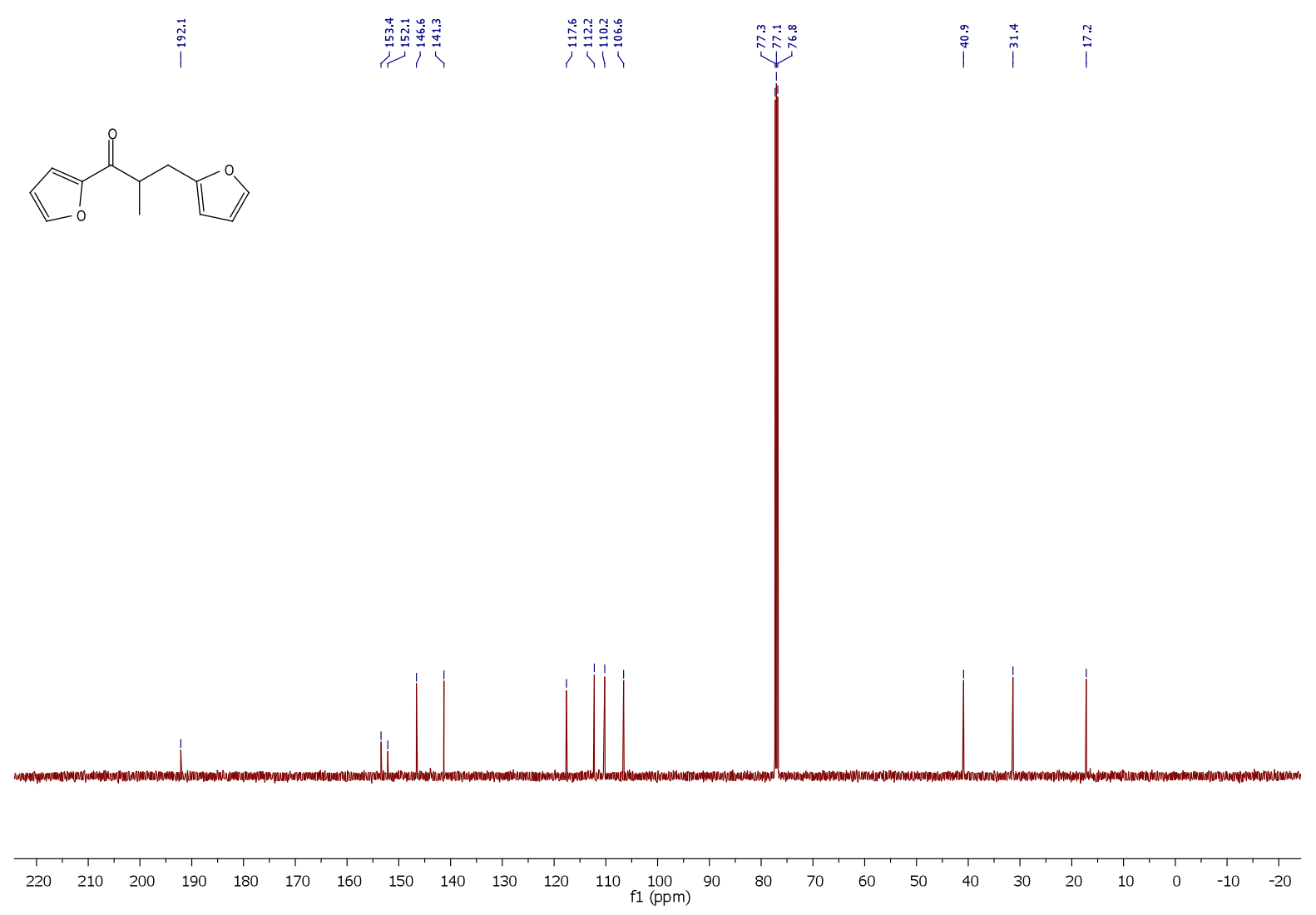

Figure S55: ${ }^{13} \mathrm{C}-\mathrm{NMR}$ of $1 \mathrm{y}$ 


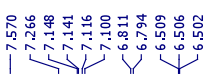

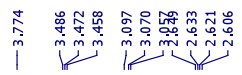

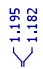
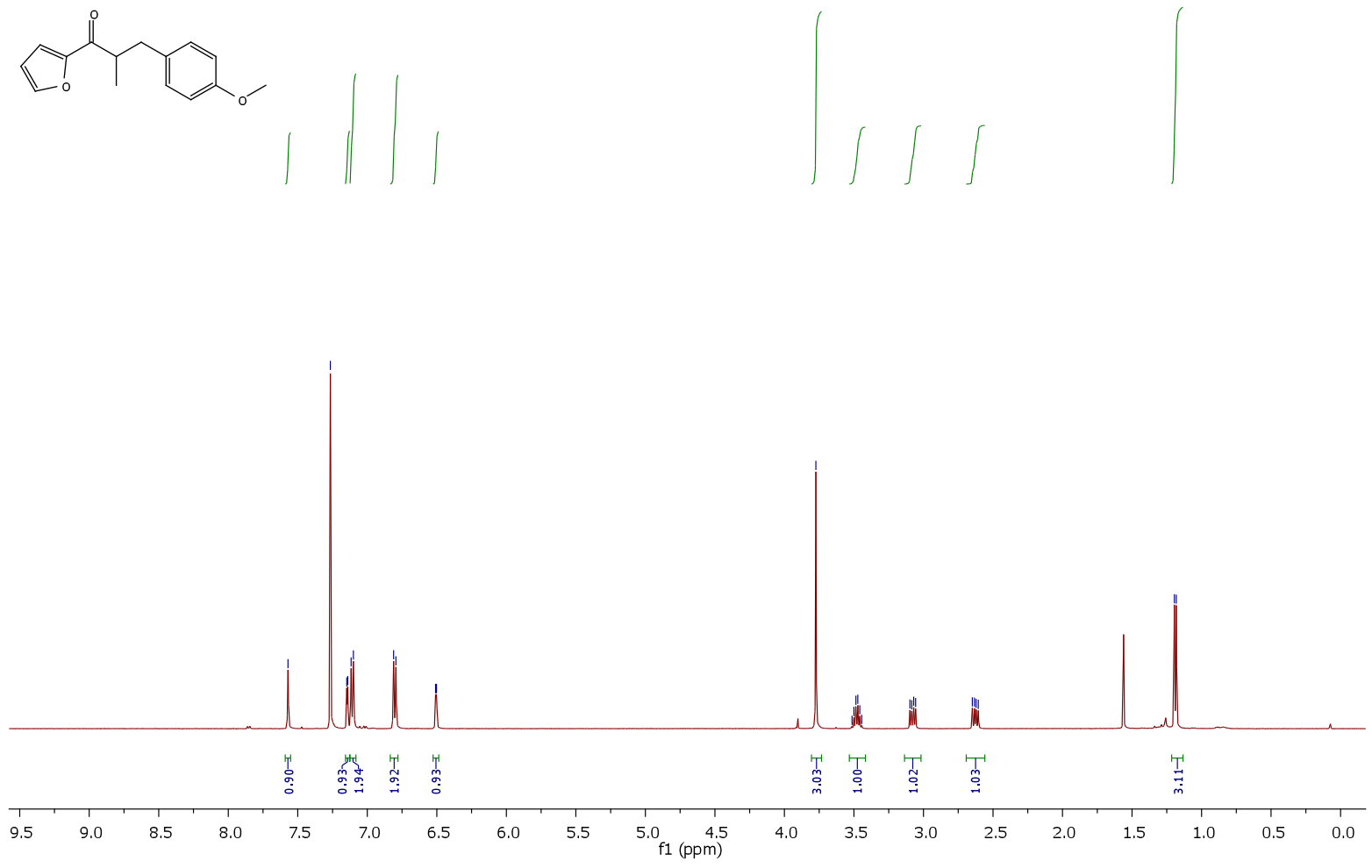

Figure S56: ${ }^{1} \mathrm{H}-\mathrm{NMR}$ of $1 \mathrm{z}$

$\stackrel{\text { Iิ }}{\stackrel{1}{2}}$

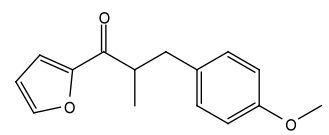

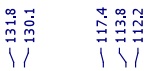

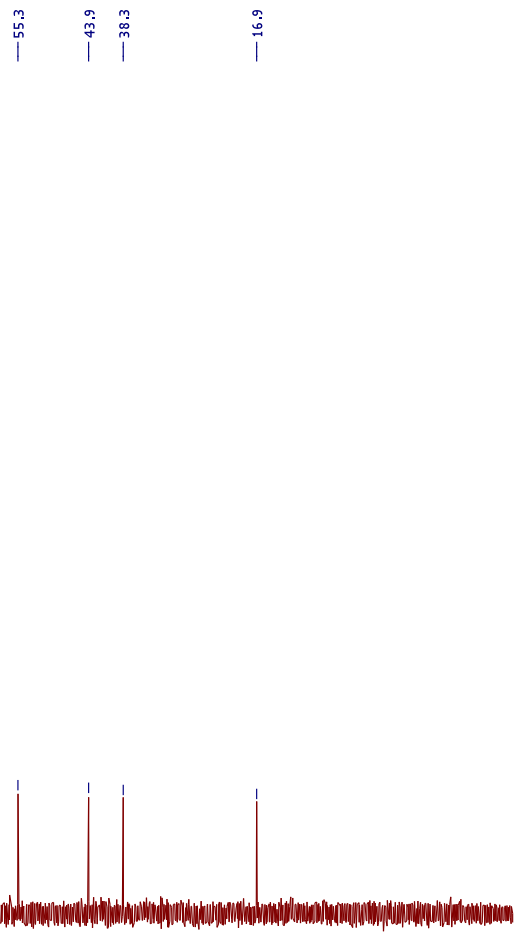

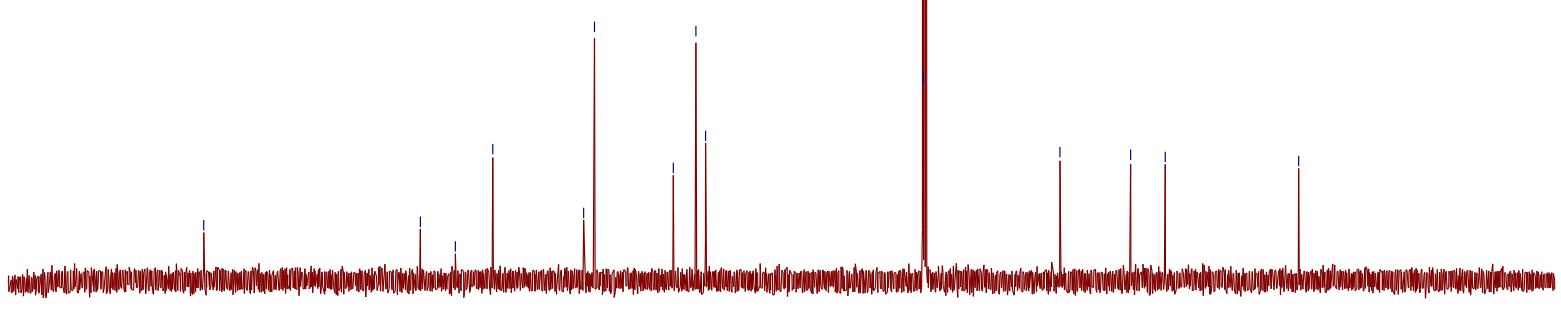

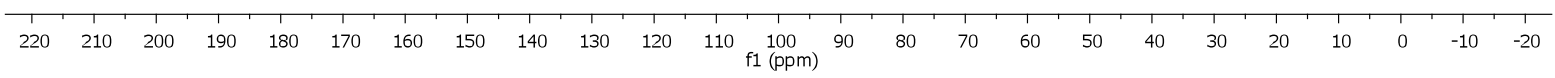

Figure S57: ${ }^{13} \mathrm{C}-\mathrm{NMR}$ of $1 \mathrm{z}$

S-51 


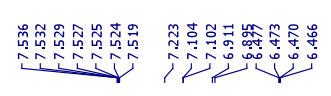

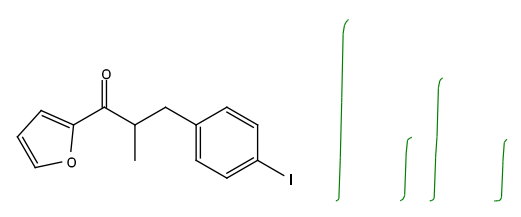

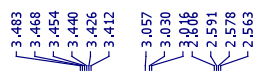

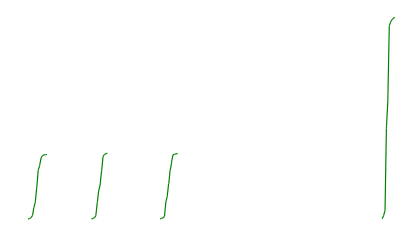

Vi

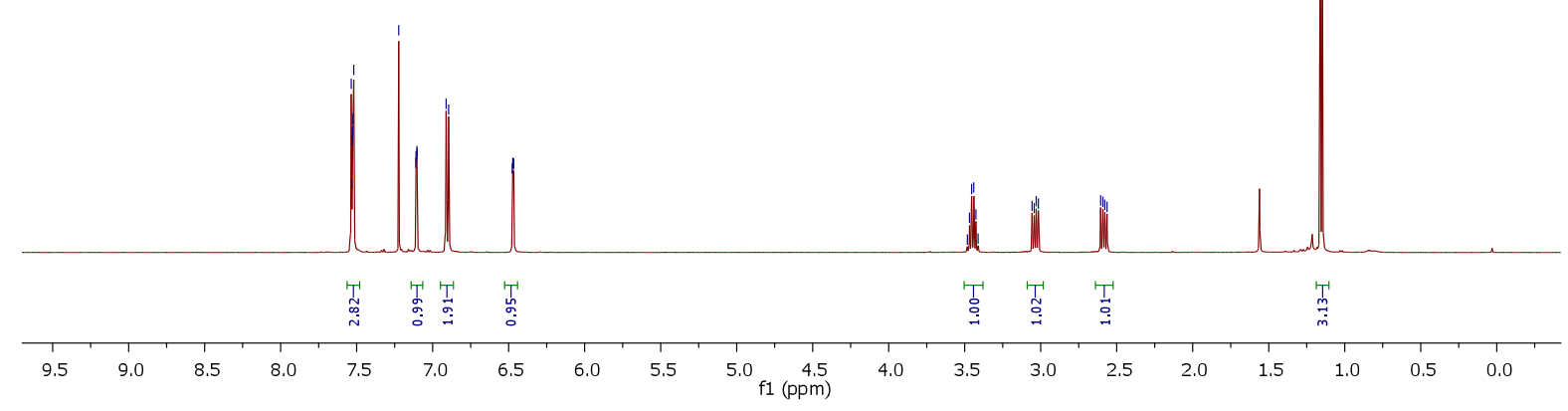

Figure S58: ${ }^{1} \mathrm{H}-\mathrm{NMR}$ of $1 \mathrm{aa}$
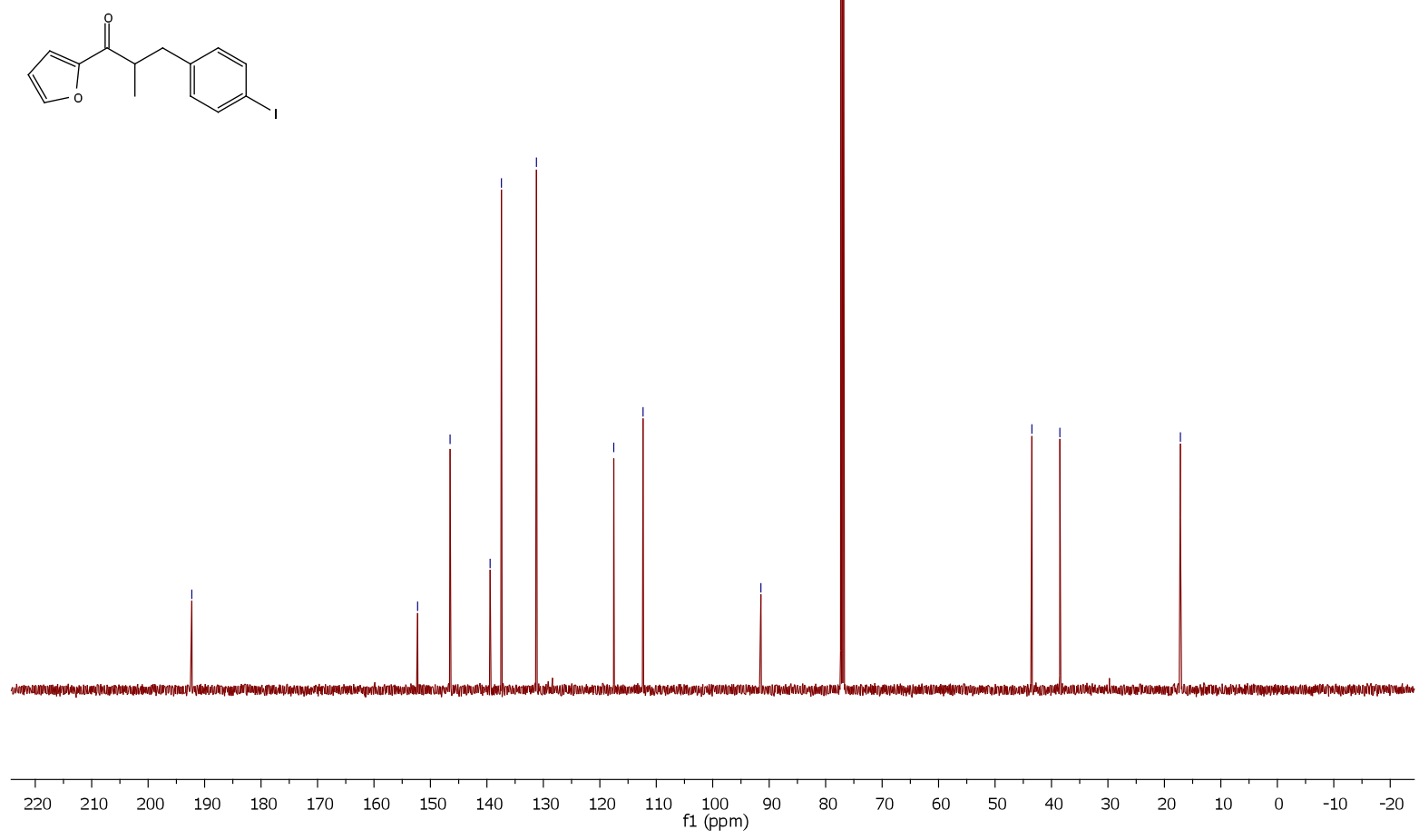

Figure S59: ${ }^{13} \mathrm{C}$-NMR of $1 \mathrm{aa}$

S-52 


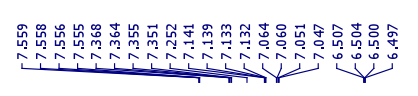
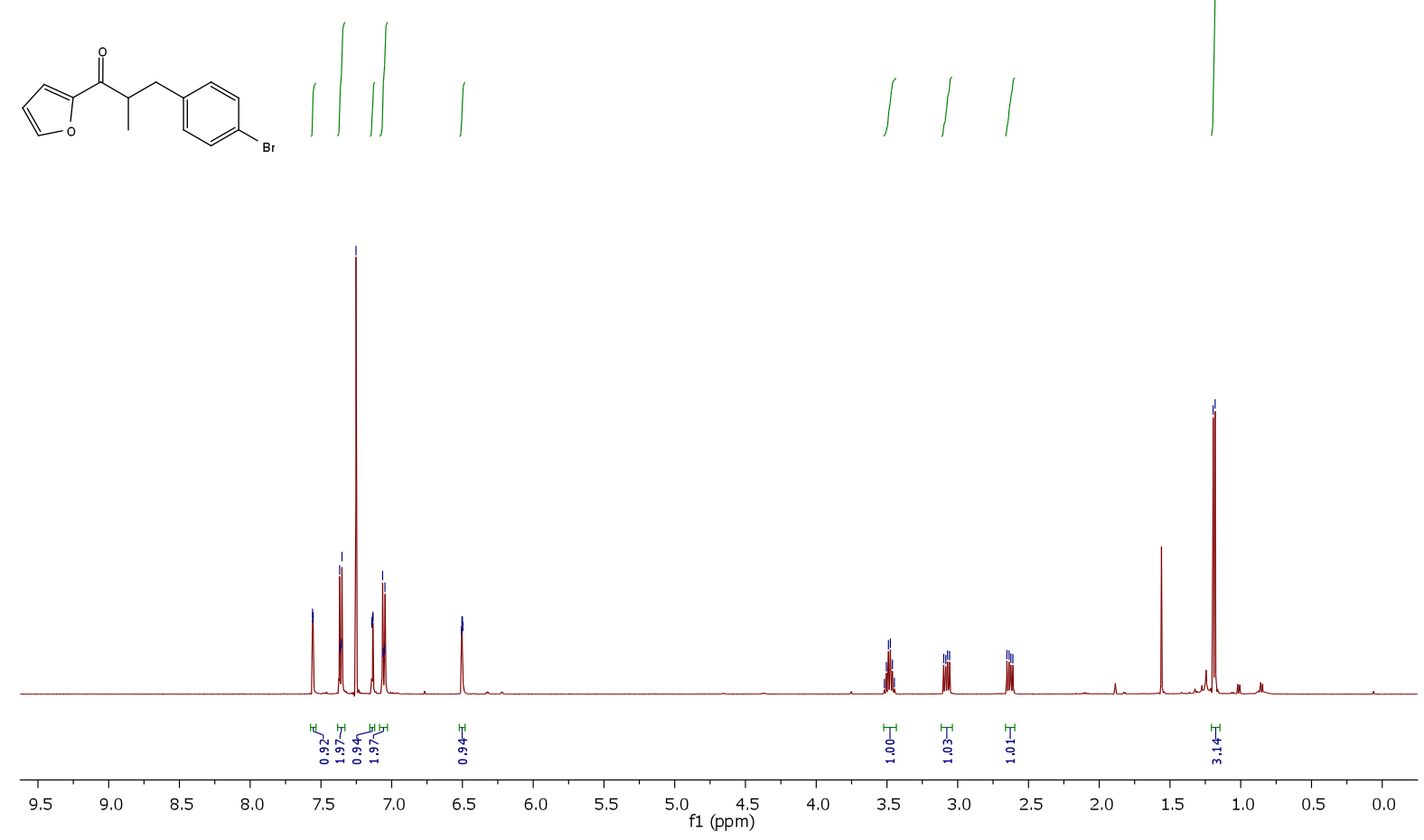

Figure S60: ${ }^{1} \mathrm{H}-\mathrm{NMR}$ of $1 \mathrm{ab}$
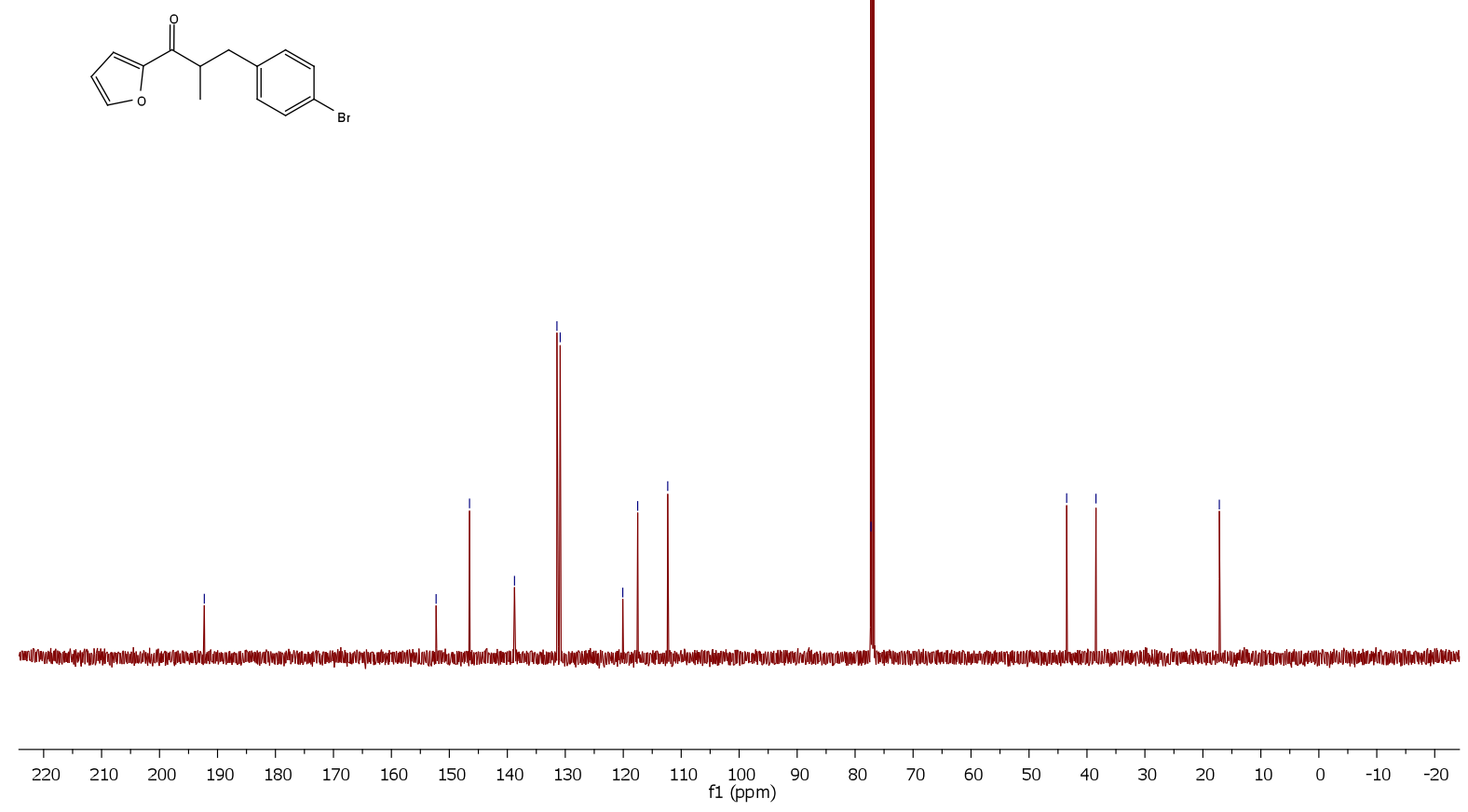

Figure S61: ${ }^{13} \mathrm{C}-\mathrm{NMR}$ of $1 \mathrm{ab}$

S-53 


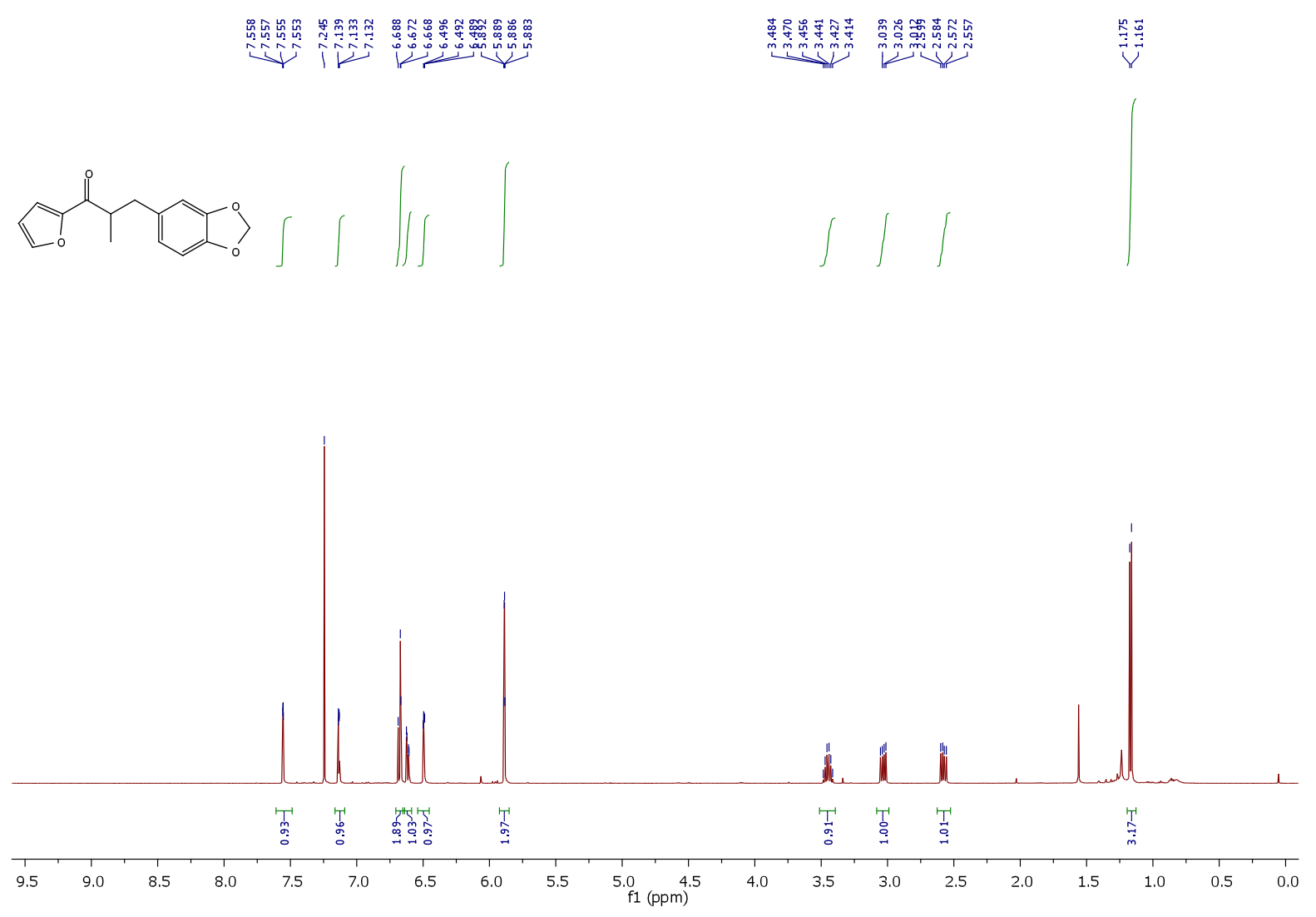

Figure S62: ${ }^{1} \mathrm{H}-\mathrm{NMR}$ of $1 \mathrm{ac}$

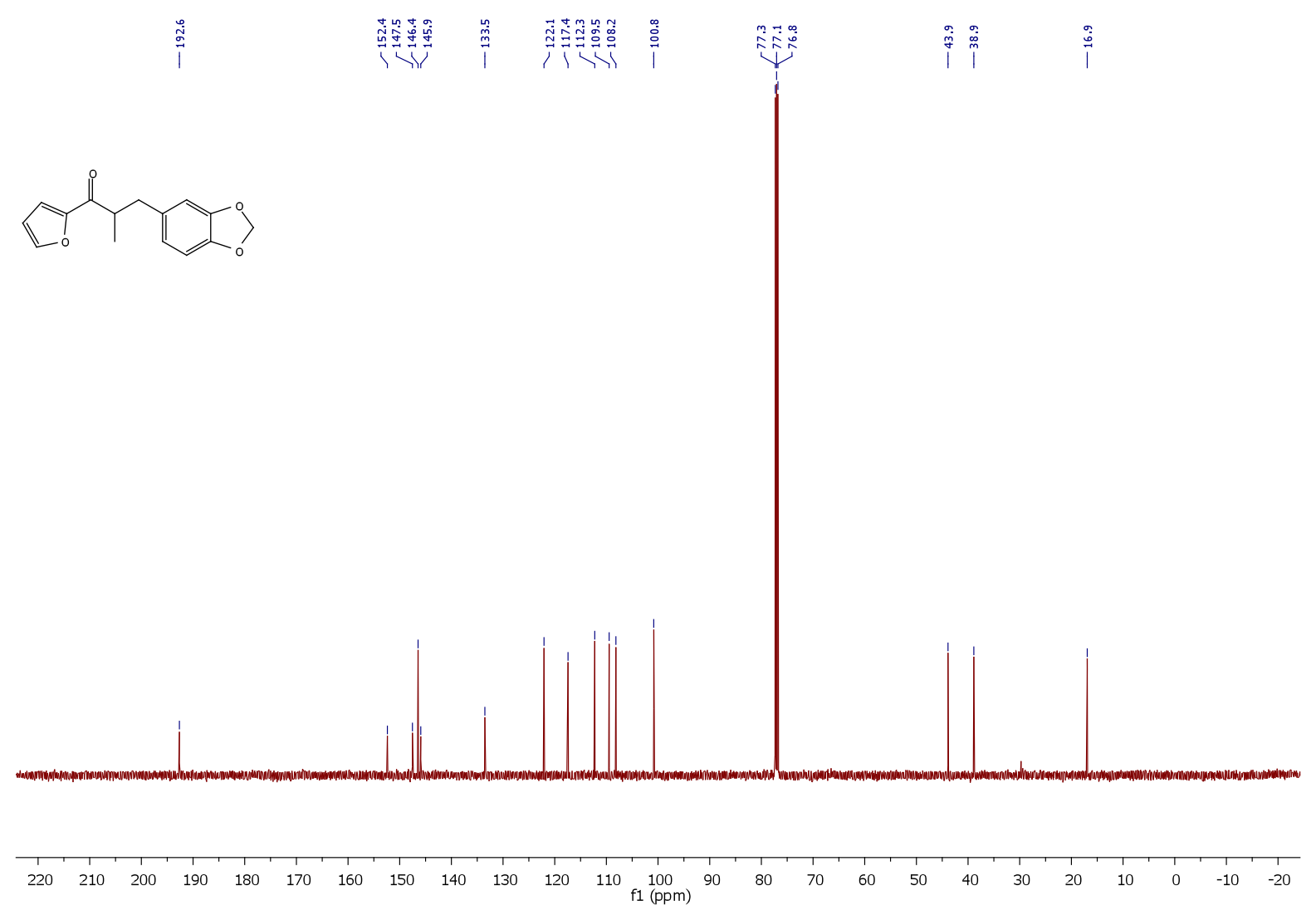

Figure S63: ${ }^{13} \mathrm{C}-\mathrm{NMR}$ of $1 \mathrm{ac}$ 


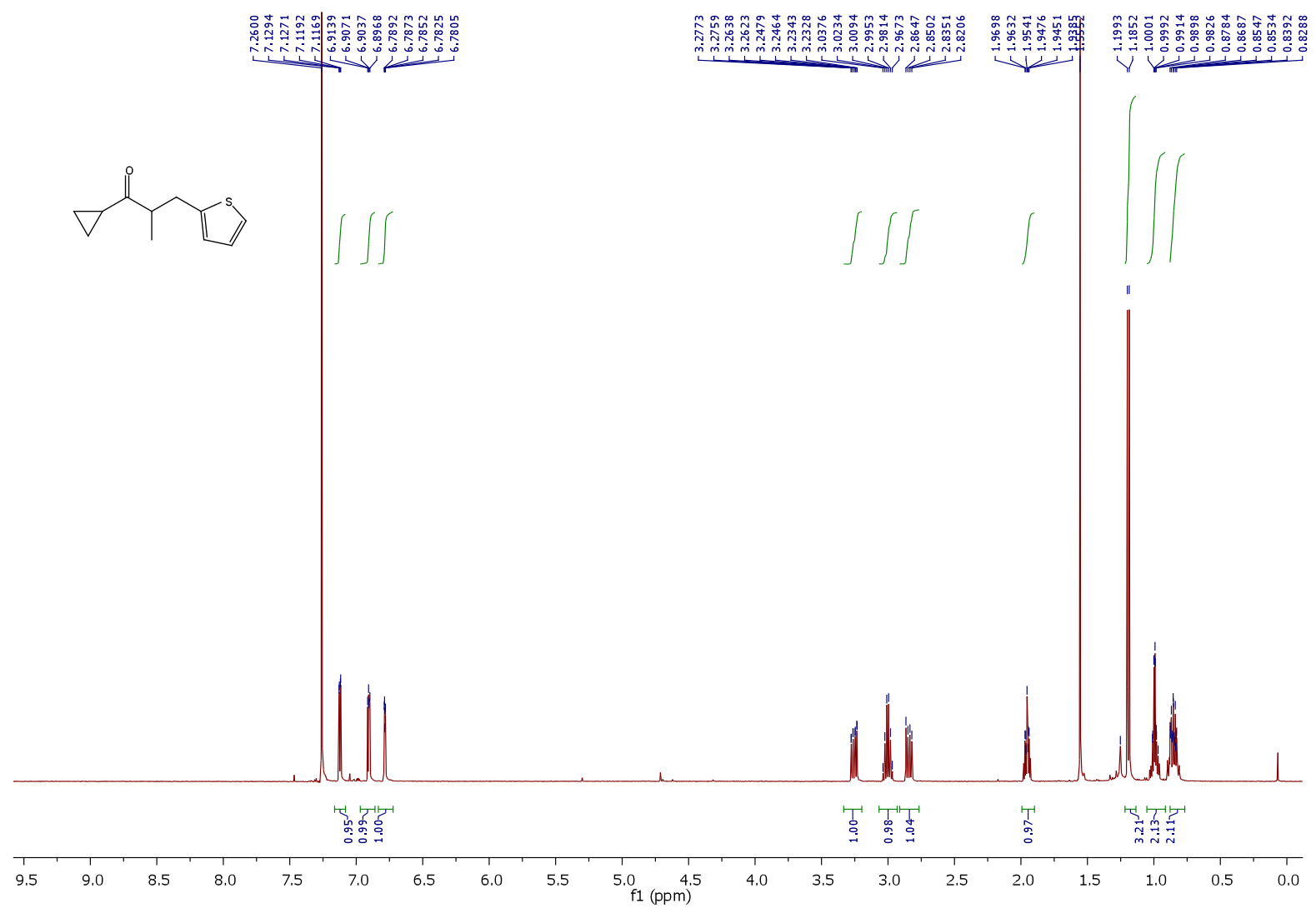

Figure S64: ${ }^{1} \mathrm{H}-\mathrm{NMR}$ of $1 \mathrm{ad}$

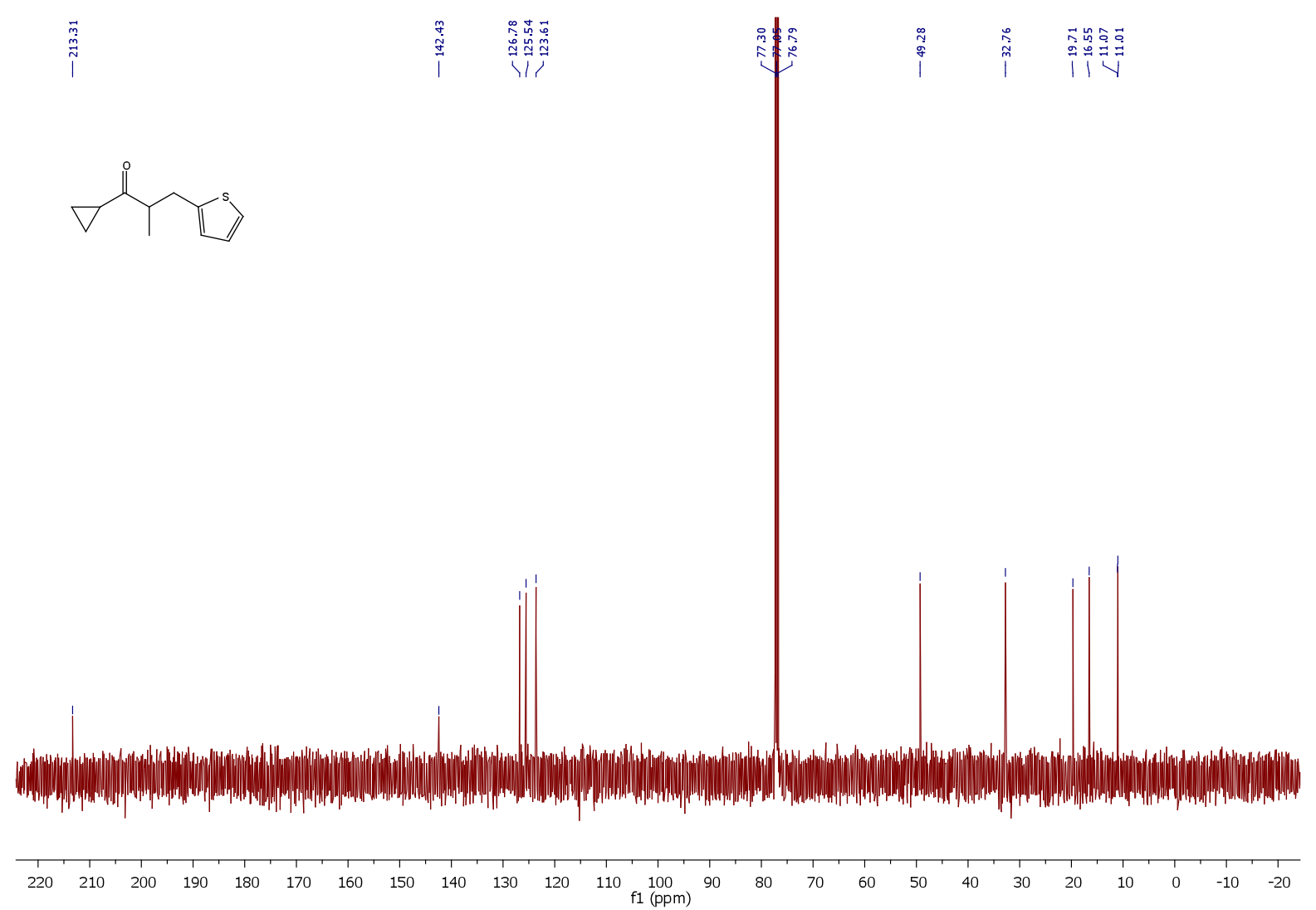

Figure S65: ${ }^{13} \mathrm{C}-\mathrm{NMR}$ of $1 \mathrm{ad}$ 


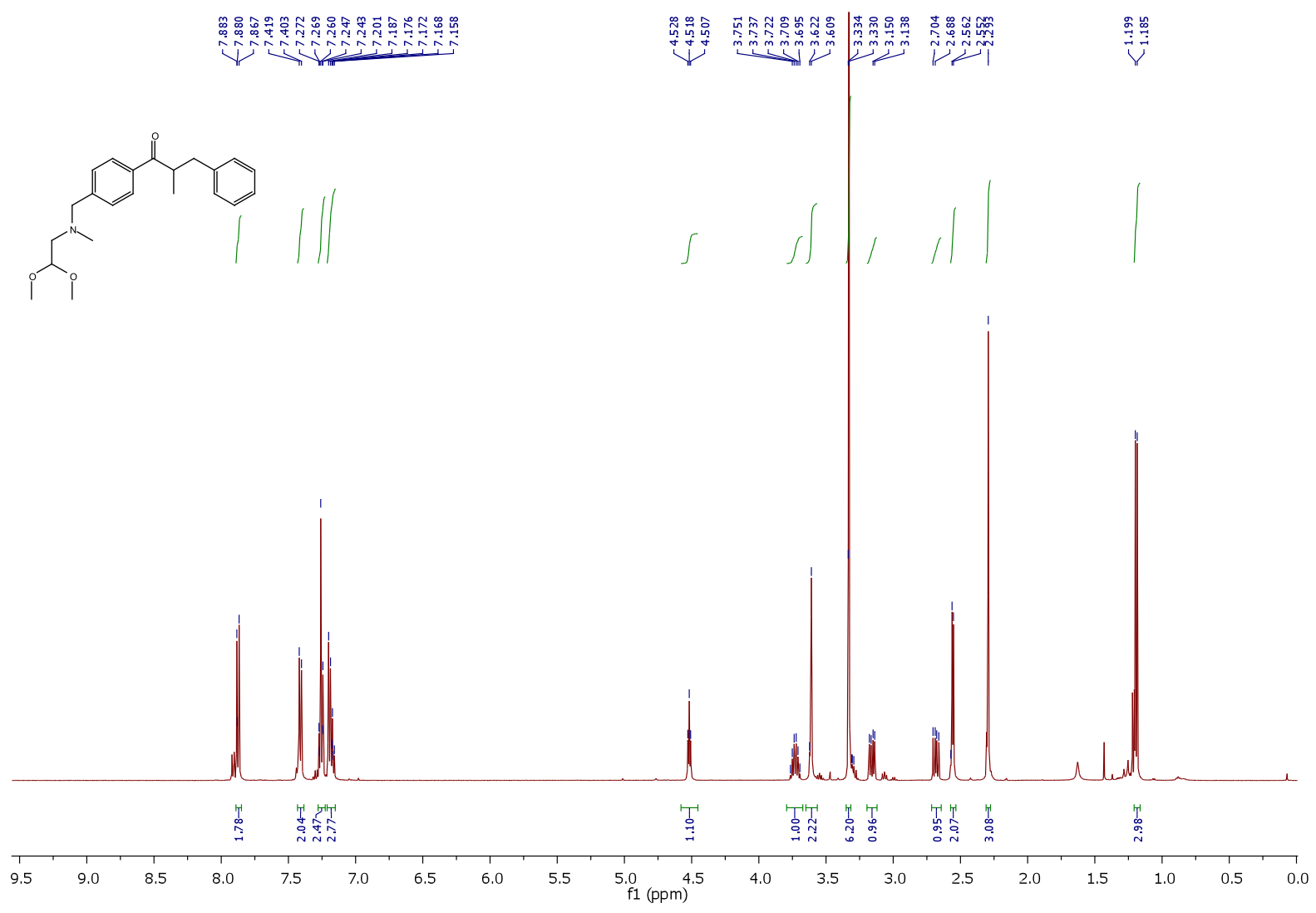

Figure S66: ${ }^{1} \mathrm{H}-\mathrm{NMR}$ of $2 \mathrm{a}$

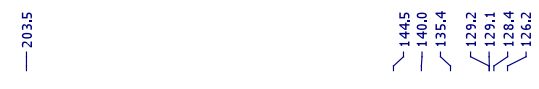

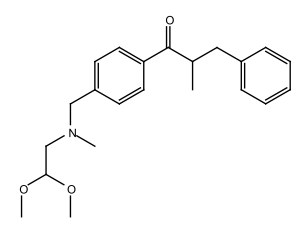

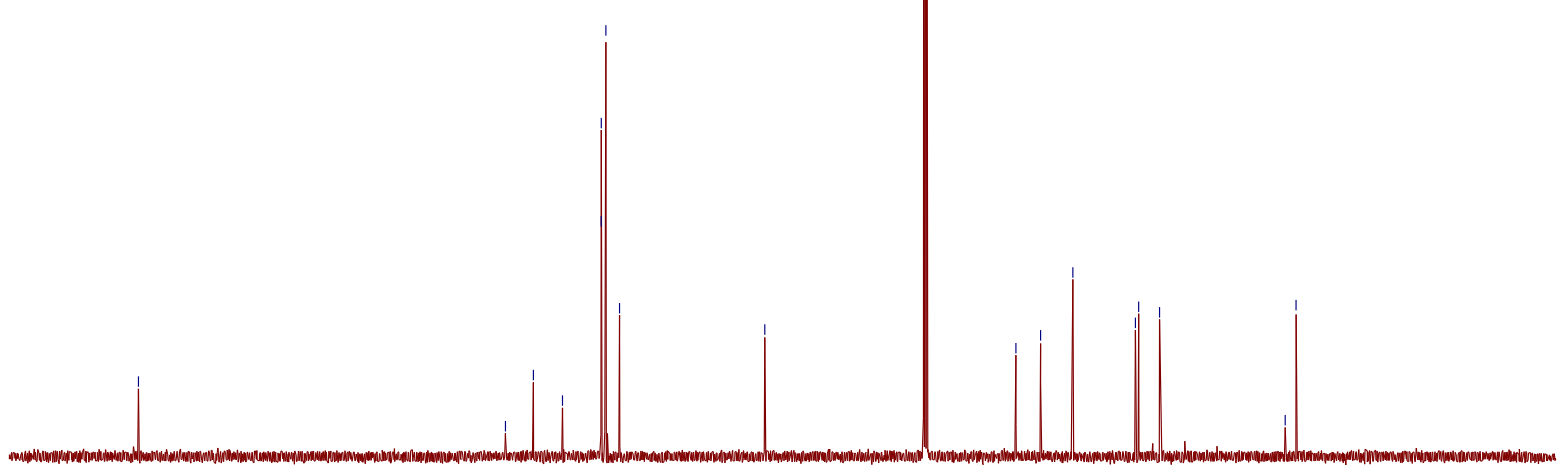

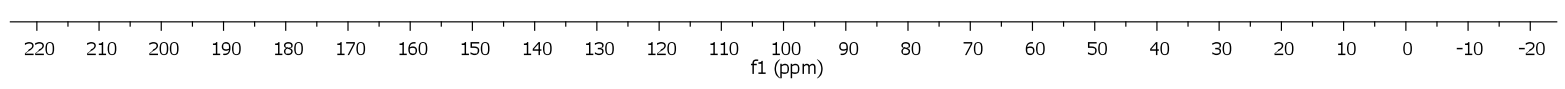

Figure S67: ${ }^{13} \mathrm{C}-\mathrm{NMR}$ of $2 \mathrm{a}$

S-56 


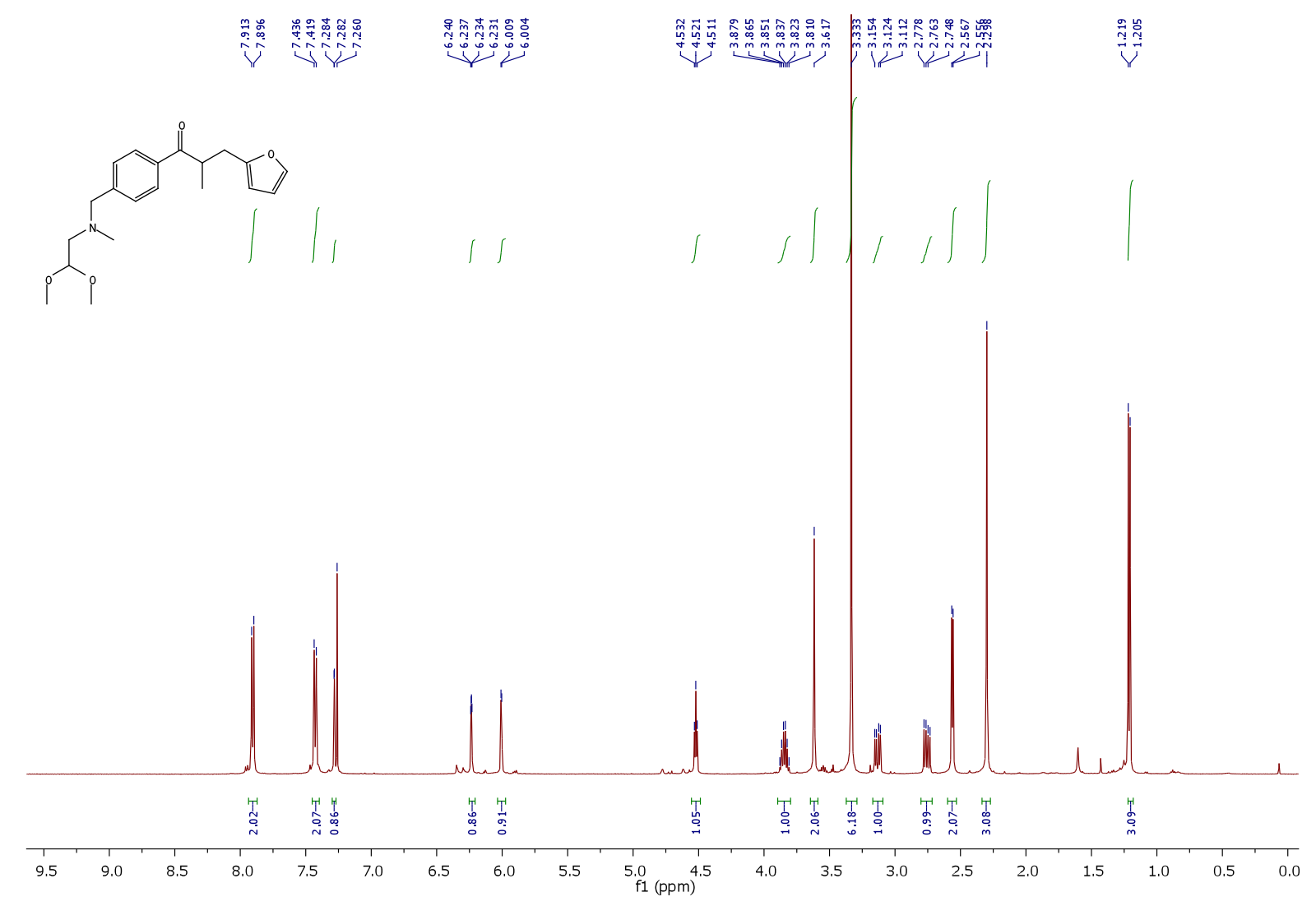

Figure S68: ${ }^{1} \mathrm{H}-\mathrm{NMR}$ of $2 \mathrm{~b}$

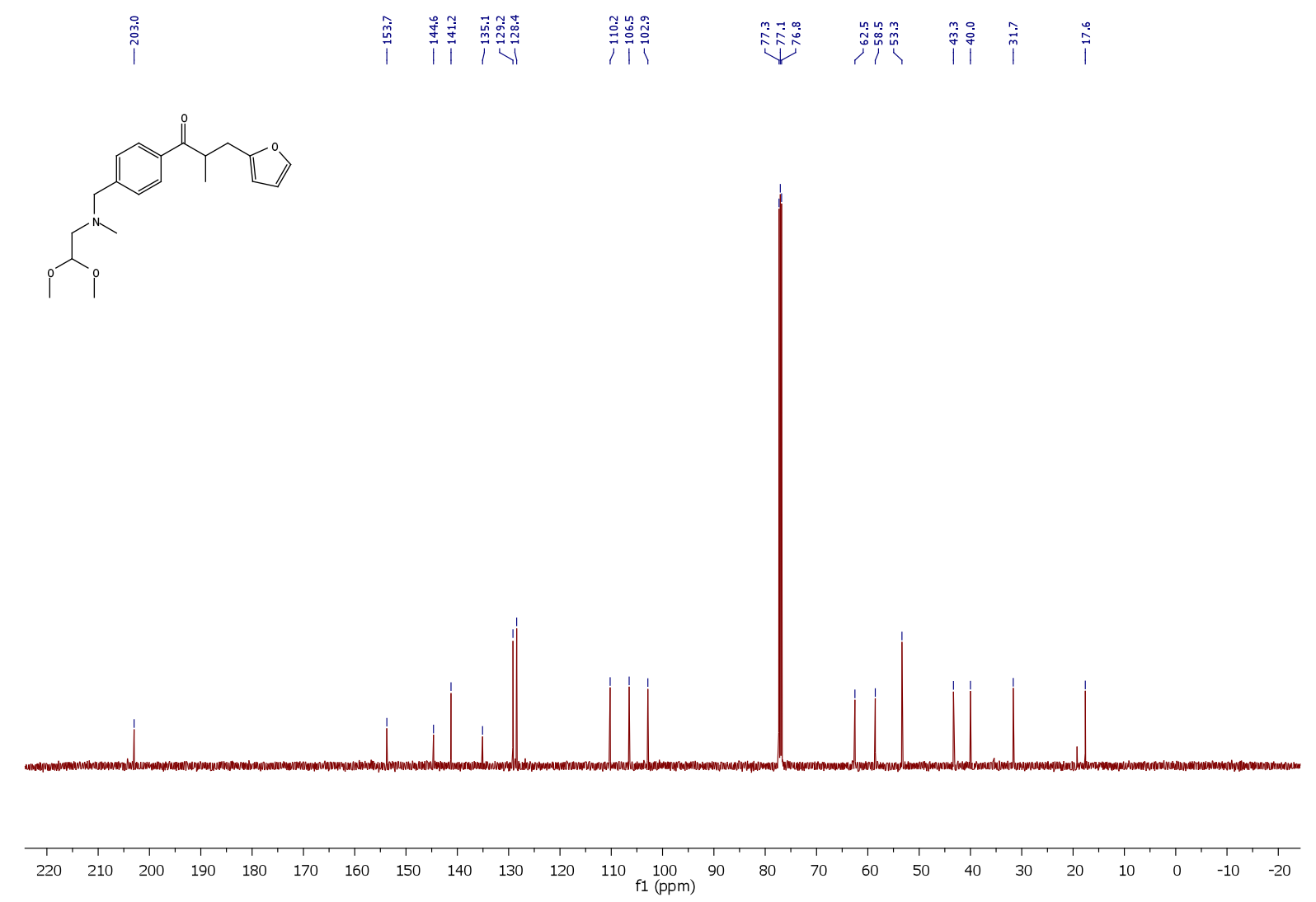

Figure S69: ${ }^{13} \mathrm{C}-\mathrm{NMR}$ of $2 \mathrm{~b}$ 


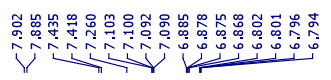
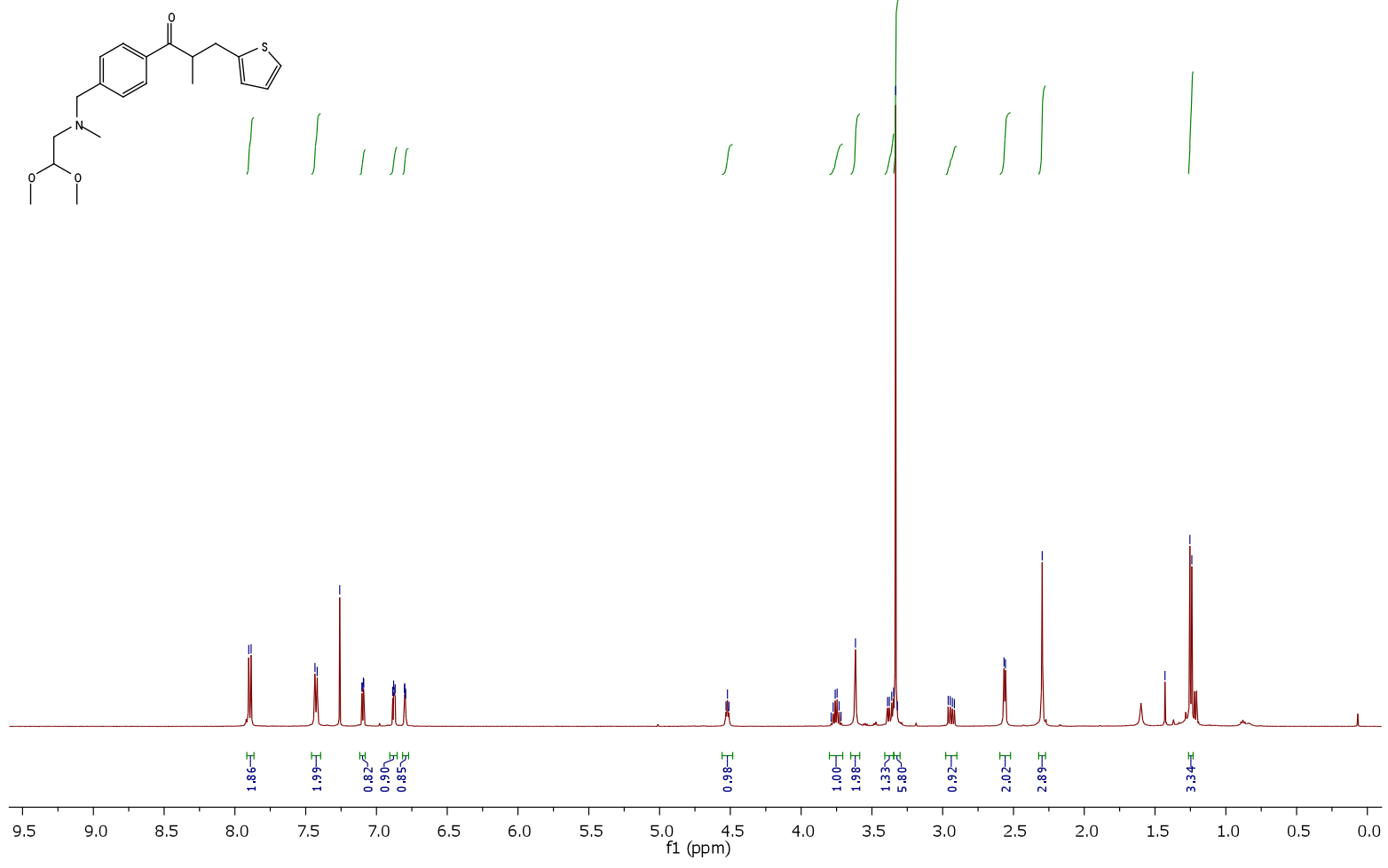

Figure S70: ${ }^{1} \mathrm{H}-\mathrm{NMR}$ of $2 \mathrm{c}$

in
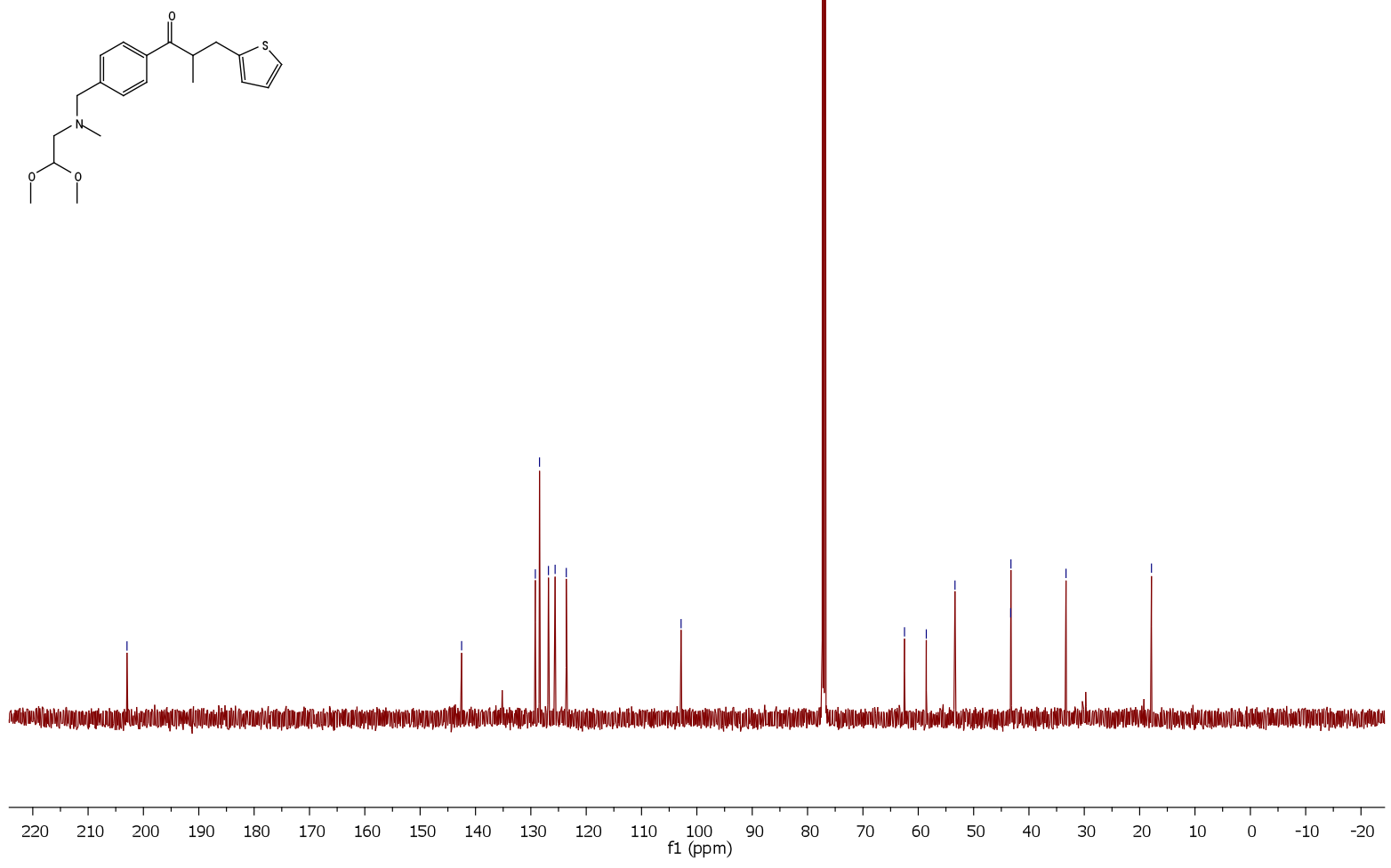

Figure $\mathrm{S} 71:{ }^{13} \mathrm{C}-\mathrm{NMR}$ of $2 \mathrm{C}$

S-58 


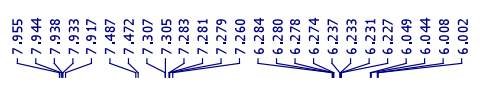

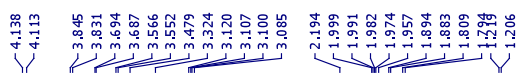
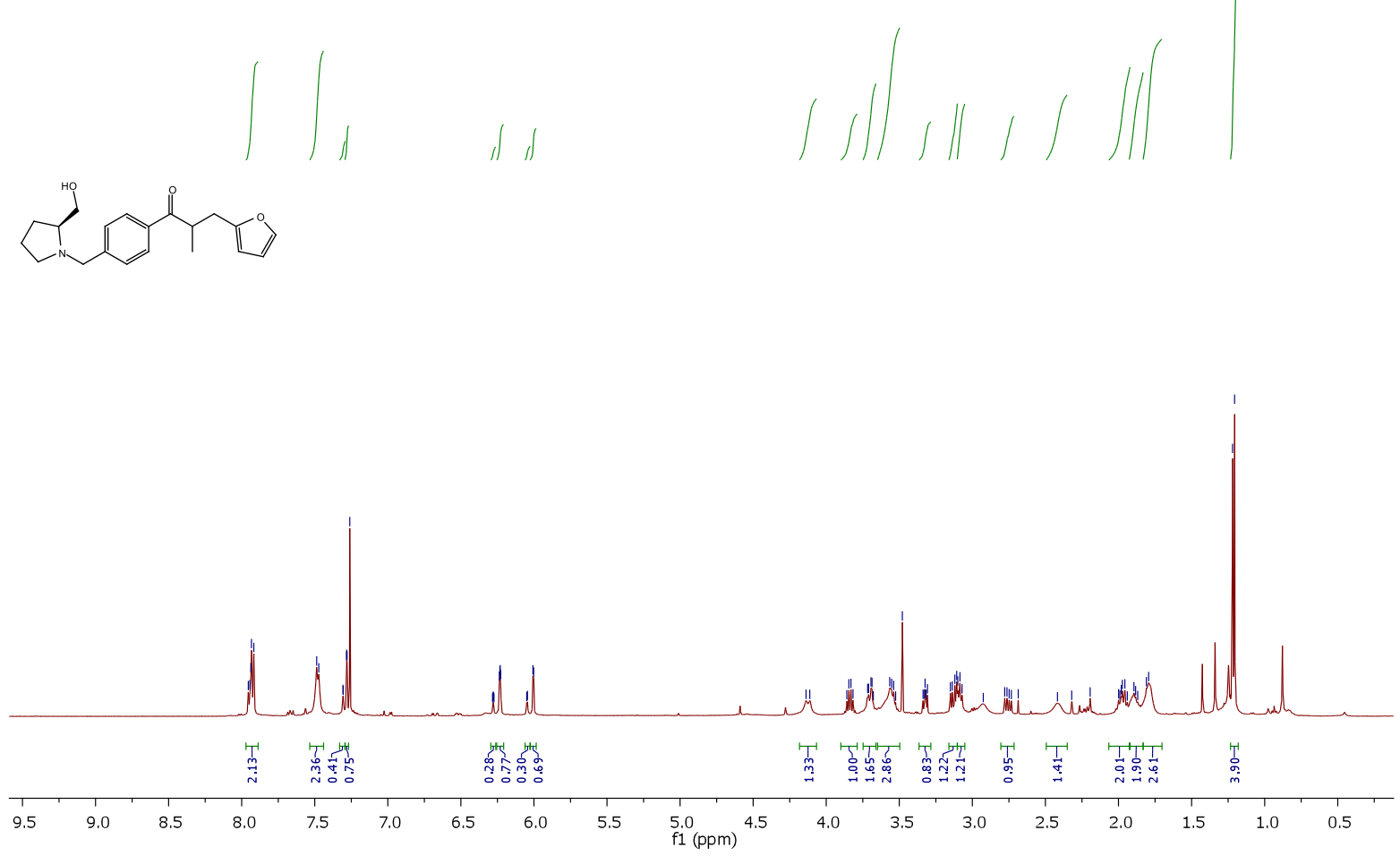

Figure S72: ${ }^{1} \mathrm{H}-\mathrm{NMR}$ of $\mathbf{2 d}$
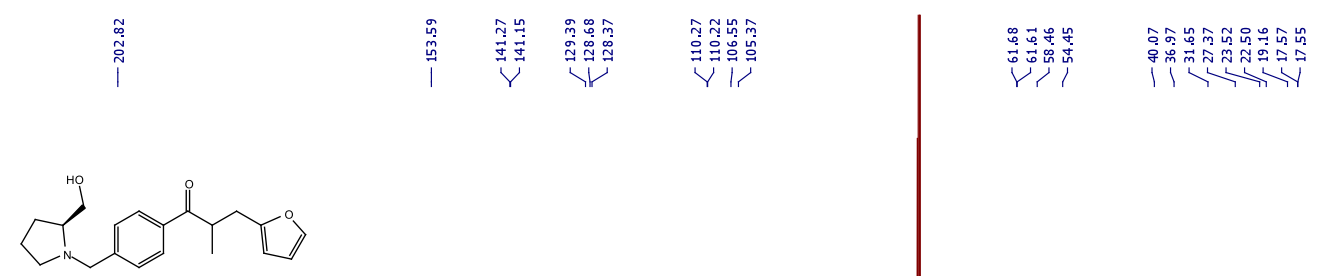

$\stackrel{\leftrightarrow}{i}$

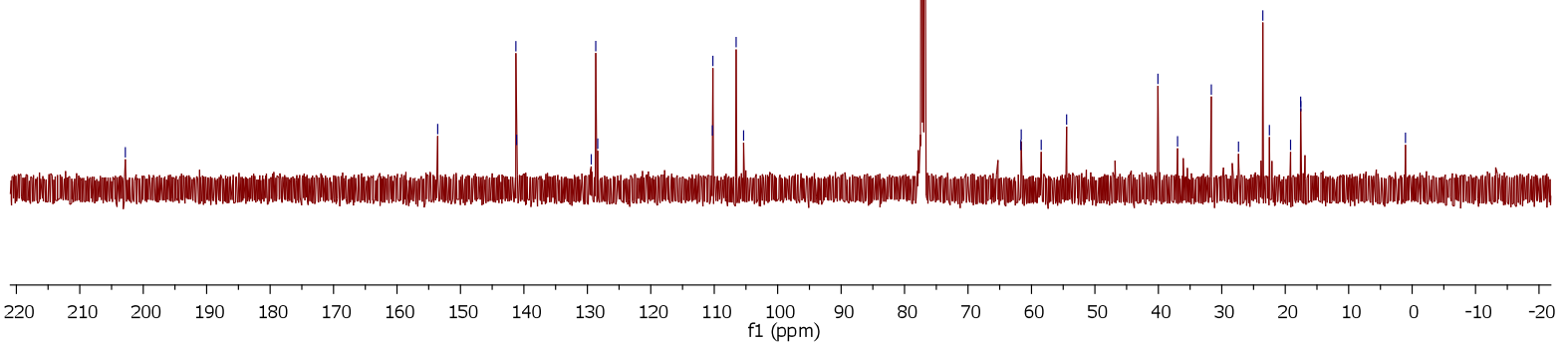

Figure S73: ${ }^{13} \mathrm{C}$-NMR of $2 \mathrm{~d}$

S-59 